\title{
WestVirginiaUniversity
}

THE RESEARCH REPOSITORY @ WVU

Graduate Theses, Dissertations, and Problem Reports

2003

\section{Characterization of point defects in nonlinear optical materials}

Madalina M. Chirila

West Virginia University

Follow this and additional works at: https://researchrepository.wvu.edu/etd

\section{Recommended Citation}

Chirila, Madalina M., "Characterization of point defects in nonlinear optical materials" (2003). Graduate Theses, Dissertations, and Problem Reports. 1917.

https://researchrepository.wvu.edu/etd/1917

This Dissertation is protected by copyright and/or related rights. It has been brought to you by the The Research Repository @ WVU with permission from the rights-holder(s). You are free to use this Dissertation in any way that is permitted by the copyright and related rights legislation that applies to your use. For other uses you must obtain permission from the rights-holder(s) directly, unless additional rights are indicated by a Creative Commons license in the record and/ or on the work itself. This Dissertation has been accepted for inclusion in WVU Graduate Theses, Dissertations, and Problem Reports collection by an authorized administrator of The Research Repository @ WVU.

For more information, please contact researchrepository@mail.wvu.edu. 


\title{
Characterization of Point Defects in Nonlinear Optical Materials
}

\author{
Madalina M. Chirila \\ Dissertation submitted to the \\ Eberly College of Arts and Sciences \\ at West Virginia University \\ in partial fulfillment of the requirements \\ for the degree of
}
Doctor in Philosophy
in
Physics

Larry E. Halliburton, PhD., Chair

Nancy C. Giles, PhD.

Mohindar S. Seehra, PhD.

Martina E. Bachlechner, PhD.

Peter M. Gannett, PhD.

Department of Physics
Morgantown, West Virginia
2003

Keywords: lithium niobate, lithium tantalate, KDP, DKDP, Gaussian 98 Calculations 


\section{ABSTRACT \\ Characterization of Point Defects in Nonlinear Optical Materials \\ Madalina M. Chirila}

Thermoluminescence (TL), optical absorption, and electron paramagnetic resonance (EPR) were used to characterize point defects in $\mathrm{LiNbO}_{3}$ and $\mathrm{LiTaO}_{3}$ crystals. A broad TL emission, peaking at $440 \mathrm{~nm}$, is observed near $94 \mathrm{~K}$ from $\mathrm{LiNbO}_{3}$ when the crystal is irradiated at $77 \mathrm{~K}$ and then rapidly warmed. From the $\mathrm{LiTaO}_{3}$ crystals two overlapping TL peaks occur at 94 and $98 \mathrm{~K}$, with each showing a 350-nm maximum in spectral emission. These peaks are observed after $77-\mathrm{K}$ exposure of the crystals to $\mathrm{x}$ rays or lasers $(266,325$, or $355 \mathrm{~nm}$ ). During excitation of these crystals at $77 \mathrm{~K}$, holes are trapped on oxygen ions adjacent to lithium vacancies and electrons are trapped on niobium and tantalum ions at regular lattice sites. These defects have characteristic EPR spectra, and the trapped electron center has an optical absorption band peaking at $1200 \mathrm{~nm}$ in $\mathrm{LiNbO}_{3}$ and $1600 \mathrm{~nm}$ in $\mathrm{LiTaO}_{3}$. Upon warming, the electrons become thermally unstable and migrate to the trapped-hole sites where radiative recombination occurs.

Optical absorption and EPR were used to characterize the production and thermal decay of point defects in $\mathrm{KD}_{2} \mathrm{PO}_{4}$. A crystal was irradiated at $77 \mathrm{~K}$ with $\mathrm{x}$ rays and then warmed to room temperature. Immediately after the irradiation broad optical absorption bands were formed at 230,390 , and $550 \mathrm{~nm}$. These bands thermally decayed in the 80 to 140 $\mathrm{K}$ range. Another absorption band near $450 \mathrm{~nm}$ appeared as the three bands disappeared. Correlations with EPR data suggest that the band at $230-\mathrm{nm}$ is associated with interstitial deuterium atoms, the two bands at 390 and $550 \mathrm{~nm}$ are associated with self-trapped holes, and the band at $450 \mathrm{~nm}$ is associated with holes trapped adjacent to deuterium vacancies.

Results from quantum-mechanical calculations performed with Gaussian 98 were correlated with hyperfine data from EPR measurements for several point defects in $\mathrm{KH}_{2} \mathrm{PO}_{4}$. The point defects modeled with calculations are: the self-trapped hole, the proton vacancy, the silicon hole, and the oxygen vacancy. Primary results from the calculations include the minimum energy, the isotropic Fermi contact coupling terms, and the lattice relaxation. 


\section{ACKNOWLEDGMENTS}

Most of all I would like to thank to my advisor, Dr. Larry Halliburton without whom this dissertation would not be possible. I deeply appreciate his continuous support, patience, and encouragement throughout my years in the department. His good will and calm will always be for me an example in life. He has also given me the chance to broaden my experience by learning new techniques and skills that will help me in the future. Working for my $\mathrm{PhD}$ was fun, and stress was never an issue in our group. This led to great research results and to good relationships within our group. I will always be grateful for all the help Dr. Halliburton has given me.

Special thanks go to Dr. Nancy Giles for many helpful discussions and encouragement over the years. I appreciate all the support she has given me allowing to use equipment from her lab whenever I needed. Her experience and knowledge of physics have proven invaluable on many occasions. She is for me an example of professionalism and integrity.

I also wish to thank Dr. Mohindar Seehra for his valuable contributions as a professor throughout the course of my studies and for serving in my committee. I have learned a great deal of physics from his classes and if I am ever going to be a professor, I will use his methods as guidelines.

I wish to thank Dr. Martina Bachlechner for her great support, and friendly attitude as a member of my committee and to Dr. Peter Gannett from Health Sciences Department for his assistance as a member of my committee.

Many thanks to Dr. Nelson Garces for his immense contribution and help on the projects we worked together. He has been a good colleague and from him I have learned a great deal about the subject of electron paramagnetic resonance. He has also provided EPR data presented in this dissertation.

I extend my appreciation to the faculty members and staff of the Physics Department and to all colleagues and friends I have worked with over my time here.

I am grateful to my family for their good thoughts and support. To my dear husband and colleague, Florin, goes my love and respect for believing, helping, and encouraging me throughout the years. 


\section{TABLE OF CONTENTS}

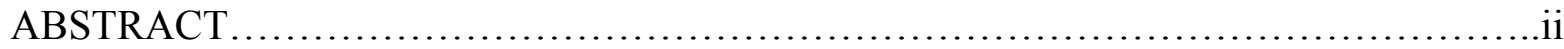

ACKNOWLEDGMENTS.....................................................

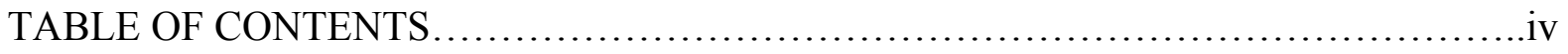

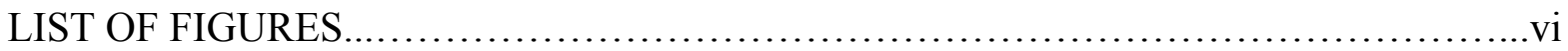

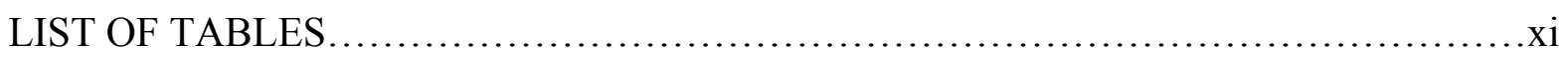

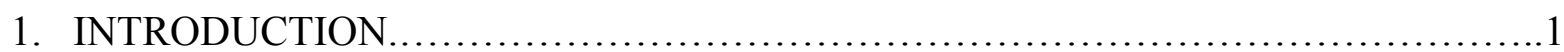

2. NONLINEAR OPTICAL MATERIALS.......................................

2.1. General description of nonlinear optical phenomena........................4

2.2. Applications of the nonlinear optical materials $\ldots \ldots \ldots \ldots \ldots \ldots \ldots \ldots \ldots \ldots \ldots$

3. CRYSTAL STRUCTURE, GROWTH, AND POINT DEFECTS........................ 12

3.1. Lithium niobate and lithium tantalate.................................. 12

3.1.1. Crystal structure..........................................12

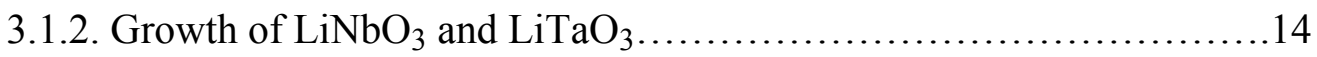

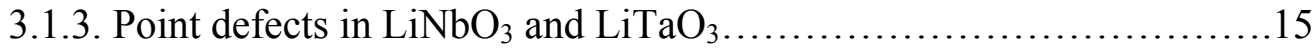

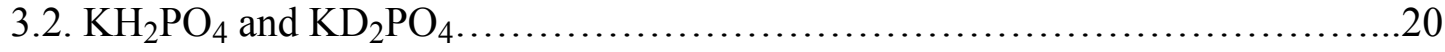

3.2.1. Crystal structure .........................................20

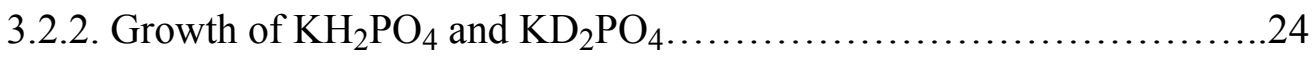

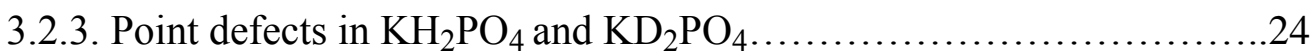

4. EXPERIMENTAL TECHNIQUES AND INSTRUMENTATION....................30

4.1. General description................................................ 30

4.2. Thermoluminescence................................................. 31

4.3. Electron paramagnetic resonance..................................... 38

4.4. Optical and infrared absorption.....................................45 


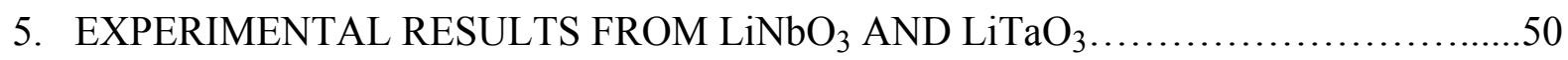

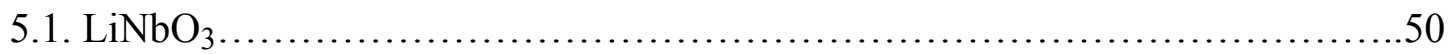

5.1.1. TL, EPR, and optical absorption results.......................50

5.1.2. Discussion and model......................................62

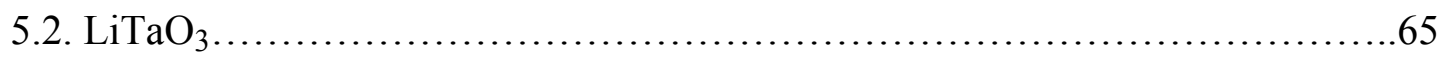

5.2.1. TL, EPR, and optical absorption results.......................65

5.2.2. Discussion and model.......................................73

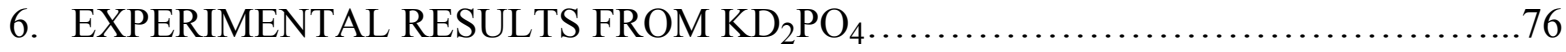

6.1. Optical absorption and EPR results................................. 76

6.2. Discussion and conclusions ........................................ 88

7. COMPUTATIONAL MODELING OF POINT DEFECTS IN $\mathrm{KH}_{2} \mathrm{PO}_{4} \ldots \ldots \ldots \ldots . . . . .92$

7.1. Technical approach............................................92

7.2. Results of the calculations and comparison with experimental data............100

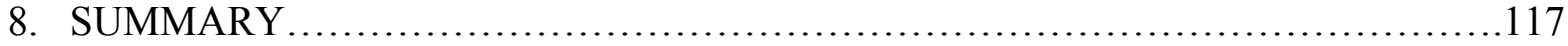

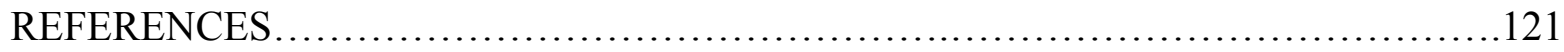




\section{LIST OF FIGURES}

Figure 3.1. Model of $\mathrm{LiNbO}_{3}$ structure in the ferroelectric phase. (a) Sequence of distorted octahedra along the polar $c$-axis. (b) Side view of the vertical planes showing the vacant octahedra. 13

Figure 3.2. Room temperature tetragonal structure of KDP. All atoms contained in one cell are illustrated by the solid lines. Phosphorus ions lie at the height marked in the figure.

Figure 3.3. Schematic diagram of ferroelectric reversal. The tetrahedra are shown in outline, with their short $\mathrm{P}-\mathrm{O}$ bonds inserted. The $\mathrm{H}$ atoms are represented by small circles, the hydrogen bonds by broken lines. The direction of the dipole is shown by the arrow. During reversal, from (i) to (ii), $\mathrm{P}$ moves to $\mathrm{P}$ ', $\mathrm{H}$ to $\mathrm{H}^{\prime}$; and oxygens while moving very little, interchange their environments. .23

Figure 4.1. Relationships between the absorption of radiation and the emission of luminescence and thermoluminescence. 32

Figure 4.2. Energy transitions involved in the production of (a) temperature independent luminescence and (b) temperature dependent emission of light .33

Figure 4.3. Simple two-level model for thermoluminescence. Allowed transitions: (1) ionization after absorption of light; (2) and (5) trapping; (3) thermal release; (4) radiative recombination and the emission of light. Electrons are the active carriers, but an exactly analogous situation arises for holes.

Figure 4.4. Setup for the thermoluminescence experiment .36

Figure 4.5. Energy-level diagram for the simplest system, as a function of applied magnetic field $H$, showing EPR absorption. $E_{\alpha}$ and $E_{\beta}$ represent the energies of $\mathrm{M}_{\mathrm{S}}=+1 / 2$ and the $\mathrm{M}_{\mathrm{S}}=-1 / 2$ .41

Figure 4.6. (a) Microwave absorption band at $\mathrm{H}=\mathrm{H}_{\mathrm{r}}$. (b) The EPR signal is the first derivative of the absorption spectrum with the center at resonant field $\mathrm{H}_{1}$

Figure 4.7. Setup for optical ultraviolet and visible absorption measurements .46 
Figure 5.1. Optical absorption from a $1 \% \mathrm{Mg}$ stoichiometric $\mathrm{LiNbO}_{3}$ crystal. These data were taken at room temperature and at $77 \mathrm{~K}$ with unpolarized light propagating along the c axis. An extrinsic absorption band, peaking near $320 \mathrm{~nm}$ and well-resolved at $77 \mathrm{~K}$, is attributed to trace amounts of $\mathrm{Fe}^{3+}$ ions.

Figure 5.2. Optical absorption from congruent and stoichiometric $\mathrm{LiNbO}_{3}$. These data were taken at room temperature with unpolarized light propagating along the $\mathrm{c}$ axis. The band edge of the congruent crystal is at longer wavelengths and the band due to Fe is not easily detected. .52

Figure 5.3. Three-dimensional view $(I-T-\lambda)$ of the thermoluminescence data taken from a $1 \% \mathrm{Mg}$ stoichiometric $\mathrm{LiNbO}_{3}$ crystal after excitation at $77 \mathrm{~K}$ with a pulsed 355-nm laser. The intensity of the emission is plotted as a function of both temperature and wavelength. .54

Figure 5.4. A cross section view (taken from the data in Figure 5.3) showing the temperature dependence of the thermoluminescence from $\mathrm{LiNbO}_{3}$. These data correspond to a wavelength of $440 \mathrm{~nm}$ (i.e., the maximum of the emission). The TL peak occurs near $94 \mathrm{~K}$ .55

Figure 5.5. A cross-section (taken from the data in Figure 5.3) showing the wavelength dependence of the thermoluminescence from $\mathrm{LiNbO}_{3}$. These data correspond to a temperature of $94 \mathrm{~K}$ (i.e., the maximum of the emission). The TL peak occurs near $440 \mathrm{~nm}$ 56

Figure 5.6. Optical absorption from the shallow electron trap, produced at $10 \mathrm{~K}$ by a $325-$ $\mathrm{nm}$ laser beam. This $1200-\mathrm{nm}$ absorption band was obtained by subtracting the spectrum taken before exposure to the laser from the spectrum taken during exposure to the laser. 58

Figure 5.7. EPR data, taken at $20 \mathrm{~K}$ with the magnetic field along the $\mathrm{c}$ axis, showing the broad trapped-hole center associated with $\mathrm{Li}$ vacancies in a $1 \% \mathrm{Mg}$ stoichiometric $\mathrm{LiNbO}_{3}$ crystal. Trace (a) was taken after the crystal was irradiated with $\mathrm{x}$ rays at $77 \mathrm{~K}$ and trace (b) was taken after the crystal was held for five min at $110 \mathrm{~K}$

Figure 5.8. EPR data, taken at $20 \mathrm{~K}$ with the magnetic field along the $\mathrm{c}$ axis, showing the high-field portion of the spectrum associated with the shallow electron trap in $\mathrm{LiNbO}_{3}$. Trace (a) was taken after the crystal was irradiated with $\mathrm{x}$ rays at 77 $\mathrm{K}$ and trace (b) was taken after the crystal was held for five min at $110 \mathrm{~K}$.....61 
Figure 5.9. Optical absorption from a VTE-treated $\mathrm{LiTaO}_{3}$ crystal. These data were taken at room temperature and at $77 \mathrm{~K}$ with unpolarized light propagating along the $\mathrm{c}$ axis. The absorption band near $300 \mathrm{~nm}$ is attributed to trace amounts of $\mathrm{Fe}^{3+}$ ions. .66

Figure 5.10. Three-dimensional view of the TL data from a VTE-treated $\mathrm{LiTaO}_{3}$ crystal that had been irradiated at $77 \mathrm{~K}$ with a pulsed 355-nm laser. The intensity of the emission is plotted as a function of both temperature and wavelength.....67

Figure 5.11. A cross-section view (taken from Figure 5.10) showing the temperature dependence of the TL from a VTE-treated $\mathrm{LiTaO}_{3}$ crystal. These data correspond to a wavelength of $350 \mathrm{~nm}$ (i.e., the maximum in the emission). Two overlapping TL peaks are observed, one near $94 \mathrm{~K}$ and one near $98 \mathrm{~K} . . . . . .68$

Figure 5.12. A cross-section view (taken from Figure 5.10) showing the wavelength dependence of the TL from a VTE-treated $\mathrm{LiTaO}_{3}$ crystal. These data correspond to a temperature of $98 \mathrm{~K}$ (i.e., the maximum of the emission). The TL peak occurs near $350 \mathrm{~nm}$.

Figure 5.13. Optical absorption from a shallow electron trap, produced at $10 \mathrm{~K}$ by a 325 $\mathrm{nm}$ laser beam. This $1600 \mathrm{~nm}$ absorption band was obtained by subtracting the spectrum taken before exposure to the laser from the spectrum taken during exposure to the laser................................................................. 70

Figure 5.14. EPR spectra from a VTE-treated $\mathrm{LiTaO}_{3}$ crystal taken (a) before irradiation, (b) after exposure to x rays at $77 \mathrm{~K}$, (c) after a five-min anneal at $100 \mathrm{~K}$, and (d) after a five-min anneal at $130 \mathrm{~K}$. The measurement temperature was $40 \mathrm{~K}$ and the magnetic field was parallel to the $\mathrm{c}$ axis. .72

Figure 6.1. Optical absorption spectra from radiation-induced point defects in DKDP. Trace a was taken at $40 \mathrm{~K}$ from the unirradiated crystal. Then the crystal was irradiated at $77 \mathrm{~K}$ with $\mathrm{x}$ rays, and traces $\mathrm{b}, \mathrm{c}, \mathrm{d}$, e, f, and $\mathrm{g}$ were subsequently taken at $80 \mathrm{~K}, 100120,140,180$, and $200 \mathrm{~K}$, respectively................78

Figure 6.2. Absorption spectrum showing only the representative optical bands at 230, 390 , and $550 \mathrm{~nm}$ formed after $\mathrm{x}$ rays irradiation and before other absorption bands are formed. This spectrum was obtained by subtracting the spectrum at

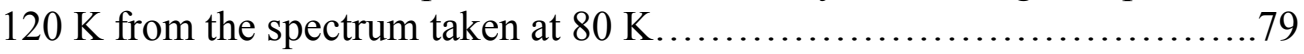

Figure 6.3. Temperature dependence of the intensity of absorption bands formed in DKDP crystal at 230,390 , and $550 \mathrm{~nm}$ after the $77 \mathrm{~K}$ irradiation 
Figure 6.4. EPR spectrum showing the $\left[\mathrm{D}_{2} \mathrm{PO}_{4}\right]^{0}$ and $\left[\mathrm{DPO}_{4}\right]^{-}$centers in a DKDP crystal after an irradiation at $77 \mathrm{~K}$ with $\mathrm{x}$ rays. These data were taken at $40 \mathrm{~K}$ with the magnetic field parallel to the $\mathrm{c}$ axis.............................. 81

Figure 6.5. EPR spectrum showing deuterium atoms $\left(\mathrm{D}^{0}\right)$ and hydrogen atoms $\left(\mathrm{H}^{0}\right)$ in a DKDP crystal after an irradiation at $77 \mathrm{~K}$ with $\mathrm{x}$ rays. These data were taken at $40 \mathrm{~K}$ with low microwave power................................8 82

Figure 6.6. Thermal anneal of the EPR spectra produced in DKDP by an irradiation with $\mathrm{x}$ rays at $77 \mathrm{~K}$. The spectra were monitored at $40 \mathrm{~K}$ after being sequentially held at each higher temperature for ten min. (a) The $\left[\mathrm{D}_{2} \mathrm{PO}_{4}\right]^{0}$ and $\left[\mathrm{DPO}_{4}\right]^{-}$ hole centers. (b) The deuterium $\left(\mathrm{D}^{0}\right)$ and hydrogen $\left(\mathrm{H}^{0}\right)$ atoms. (c) $\mathrm{A}\left(\mathrm{PO}_{3}\right)^{2-}$ oxygen-vacancy center...............................................

Figure 6.7. EPR spectrum showing an oxygen-vacancy $\left(\mathrm{PO}_{3}\right)^{2-}$ center in a DKDP crystal that had first been irradiated at $77 \mathrm{~K}$ with $\mathrm{x}$ rays and then held at $200 \mathrm{~K}$ for ten min. These data were taken at $40 \mathrm{~K}$ with the magnetic field parallel to the $\mathrm{c}$ axis............................................................. 86

Figure 6.8. EPR spectrum showing the $\left(\mathrm{PO}_{3}\right)_{\mathrm{B}}{ }^{2-}$ and $\left(\mathrm{PO}_{3}\right)_{\mathrm{D}}{ }^{2-}$ electron centers and the silicon-associated hole center in a DKDP crystal that had first been irradiated at $77 \mathrm{~K}$ with $\mathrm{x}$ rays and then held for $18 \mathrm{~h}$ at room temperature. These data were taken at room temperature with the magnetic field parallel to the $\mathrm{c}$ axis.

Figure 7.1. A 3D view of the $\left(\mathrm{H}_{16} \mathrm{P}_{5} \mathrm{O}_{20}\right)$ cluster. The $\mathrm{c}$ axis is perpendicular to the plane of the paper. Phosphorous 1 (P1) is in the plane of the paper, whereas P10 and P18 are above this plane and P26 and P34 are below. In the central $\mathrm{PO}_{4}$ unit, $\mathrm{O} 2$ and $\mathrm{O} 3$ are above $\mathrm{P} 1$ and $\mathrm{O} 4$ and $\mathrm{O} 5$ are below 101

Figure 7.2. KDP tetragonal structure. In this 3D view one $a$ axis is perpendicular to the plane of the paper. The other $a$ axis and c axis are in the plane of the paper. $\mathrm{PO}_{4}$ units having P26 and P34 in the center are superimposed in this view due to the symmetry of the crystal. 102

Figure 7.3. A three-dimension view of the central $\mathrm{PO}_{4}$ unit with the adjacent hydrogen atoms showing the change in the position of the four atoms $\mathrm{O} 2, \mathrm{O} 3, \mathrm{H} 6$, and $\mathrm{H} 8$ after the geometry optimization. O2', O3', H6', and $\mathrm{H} 8$ ' represent the atoms at the new positions after the geometry optimization. .106 
Figure 7.4. A three-dimensional view of the central $\mathrm{PO}_{4}$ unit missing one hydrogen atom in order to model the proton vacancy point defect. This is a before and after the geometry optimization representation. The atoms at the new positions are labeled with primes............................................... 111

Figure 7.5. A three-dimensional view of the $\mathrm{SiO}_{4}$ central unit in $\mathrm{KDP}$, showing the "before" and "after" positions of the atoms...............................114 


\section{LIST OF TABLES}

Table 2.1. Selected optical properties of the NLO crystals in this study.........................9

Table 3.1. Selected physical properties of $\mathrm{LiNbO}_{3}$ and $\mathrm{LiTaO}_{3}$ crystals.....................12

Table 3.2. Selected physical properties of KDP and KD*P crystals............................20

Table 7.1. The basic structure of a Gaussian 98 input file.........................................95

Table 7.2. Summary of Gaussian results for the self-trapped hole center in $\left(\mathrm{H}_{16} \mathrm{P}_{5} \mathrm{O}_{20}\right)$ cluster. For comparison purposes, the experimental results give a Fermi contact value of approximately $30 \mathrm{G}$ for the primary phosphorus hyperfine interaction and $3.2 \mathrm{G}$ for the hyperfine interaction with one proton............108

Table 7.3. Fermi contact coupling terms resulted from the geometry optimization of the Si-hole center.....................................................113

Table 7.4. Summary of Gaussian results for a $\left(\mathrm{PO}_{3}\right)^{2-}$ center in KDP. For comparison, the isotropic Fermi contact value from EPR experiment is $637 \mathrm{G}$ for the interaction with the phosphorus (in one experimentally observed

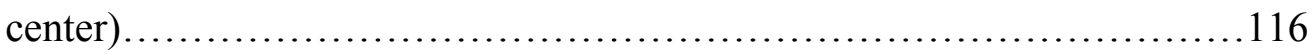




\section{CHAPTER 1}

\section{INTRODUCTION}

The nonlinear optical materials included in this study are lithium niobate $\left(\mathrm{LiNbO}_{3}\right)$, lithium tantalate $\left(\mathrm{LiTaO}_{3}\right)$, potassium dihydrogen phosphate $\left(\mathrm{KH}_{2} \mathrm{PO}_{4}\right.$ or $\left.\mathrm{KDP}\right)$, and deuterated potassium dihydrogen phosphate $\left(\mathrm{KD}_{2} \mathrm{PO}_{4}\right.$ or $\left.\mathrm{KD} * \mathrm{P}\right)$. These crystals belong to a select group of ferroelectric materials that have found important applications in many areas of advanced technology where optics and lasers dominate.

The electro-optic, photorefractive, and nonlinear optical properties of lithium niobate and lithium tantalate crystals have been widely studied for more than three decades. The great interest in these compounds is mainly due to their use in frequency conversion and modulator devices. $\mathrm{LiNbO}_{3}$ and $\mathrm{LiTaO}_{3}$ are often used as angle-tuned second harmonic generators for laser beams with wavelength greater than $1 \mu \mathrm{m}$, as optical parametric oscillators with output in the mid-infrared when pumped at $1.064 \mu \mathrm{m}$, and as quasi-phasematched (i.e., periodically poled) structures to generate doubled beams. Due to the large electro-optic and acousto-optic coefficients, $\mathrm{LiNbO}_{3}$ crystals are the most commonly used material for Pockels cells, Q-switches, phase modulators, and surface acoustic wave filters $[1,2]$. Lithium tantalate is also of interest for these same applications because of its large nonlinear optical coefficients and increased photorefractive damage resistance. Applications of $\mathrm{LiTaO}_{3}$ include surface acoustic wave devices, holographic storage, channel waveguides, periodically poled structures, and electro-optic deflectors [3].

There are many optical devices that can be constructed from niobate and tantalate crystals. These include high-speed all-optical switches, periodically poled frequency converters, high-density bulk holographic storage memories, and fast-response optical limiters. Initially, attention was focused on $\mathrm{LiNbO}_{3}$ and $\mathrm{LiTaO}_{3}$ crystals pulled from 
congruent melts, but significant improvements in growth techniques have now shifted much of the recent activity to stoichiometric crystals and high purity crystals. During the lengthy evolution of $\mathrm{LiNbO}_{3}$ and $\mathrm{LiTaO}_{3}$ as device materials, the nature and behavior of point defects in these materials have remained an unsolved problem.

In spite of the intense efforts to minimize the presence of impurities and defects, these crystals are still slightly Li-deficient and, as a result, most of the impurities and defects will occupy Li sites. Even excess $\mathrm{Nb}$ occupies vacant Li sites in lithium niobate. This results in $\mathrm{Nb}$ antisite defects (i.e., $\mathrm{Nb}_{\mathrm{Li}}$ centers). Other known defects formed during growth are oxygen vacancies and hydroxyl molecular ions. A common impurity found in these crystals is Fe which is known to have a strong influence on the visible optical absorption and on the photorefractive effect ("laser damage").

Potassium dihydrogen phosphate (KDP) and potassium deuterium phosphate (KD*P or DKDP) are commercially available materials with numerous laser-based applications. These crystals exhibit excellent electro-optical and nonlinear optical properties and are commonly used in frequency conversion applications such as second, third, and fourthharmonic generation and in electro-optical modulation. Easy growth of large single crystals, a broad transparency range, a high optical damage threshold, and relatively low production cost are all qualities that make these phosphate crystals attractive for and well suited to a variety of optical applications.

In nearly every case, however, these applications are limited by the presence of a variety of optically active point defects. A large number of these unwanted defects have already been investigated in this set of materials, although many which have important links to device performance are still not well understood. Developing a more comprehensive understanding of the point defects in these materials may provide useful feedback for crystal growers and may lead to improved device performance.

The goal of the present study is to identify and characterize optically active point 
defects in these crystals. Using results from thermoluminescence, optical absorption, and electron paramagnetic resonance experiments, I describe how the point defects can be recognized and what are the possible models and mechanisms that can explain the nature and behavior of these point defects.

This dissertation is organized in the following way. Chapter 2 discusses the origin of nonlinear optical phenomena. In Chapter 3, some of the physical properties of these four crystals are presented. Chapter 3 also provides a review of some of the previous research done on these materials by other investigators. In Chapter 4, the experimental equipment and procedures used in this project are described. Chapter 5 contains results from stoichiometric $\mathrm{LiNbO}_{3}$ and $\mathrm{LiTaO}_{3}$ crystals. In Chapter 6, results from experiments on KDP and DKDP are presented. Finally, Chapter 7 of this dissertation focuses on the computational modeling of point defects in KDP and shows the good agreement obtained with experimental data. 


\section{CHAPTER 2}

\section{NONLINEAR OPTICAL MATERIALS}

\subsection{General description of nonlinear optical phenomena}

There are several physical properties of the materials selected for this study that are very important for their use in nonlinear optical devices. These include the nonlinear susceptibility, the birefringence, the nonlinear optical coefficients, and the electro-optic coefficient.

Generally, the field of nonlinear optics (NLO) is understood to encompass those phenomena in which the electric polarization strongly depends on the higher-order terms in a power expansion of the electric field intensity, i.e., it does not just represent a simple linear dependence on the electric field intensity. This chapter offers basic information about the nonlinear optical phenomena and the related physical properties of the materials included in this study.

Second harmonic generation (SHG) was the first nonlinear optical effect ever observed in which a coherent input generated a coherent output. But nonlinear optics covers a much broader scope. It deals in general with the nonlinear interaction of light with matter and includes problems such as light-induced changes of the optical properties of a medium. Each nonlinear optical process consists of two steps. The intense light first induces a nonlinear response in a medium, and then the medium in reacting modifies the optical fields in a nonlinear way. Other NLO effects are third, fourth, and fifth harmonic generation, optical parametric oscillation, linear and quadratic electro-optic effects, acousto-optic effect, stimulated Raman scattering, and self-focusing of light [4].

The electric field of a light wave propagating through a medium exerts forces on the 
loosely bound outer, or valence, electrons. Ordinarily these forces are quite small, and in a linear isotropic medium the resulting electric polarization is parallel with and directly proportional to the applied electric field. In effect, the polarization follows the field; if the latter is harmonic, the former will be harmonic as well [1,4]. Consequently, one can write

$$
P=\varepsilon_{0} \chi E
$$

where $\varepsilon_{0}$ is the permittivity of free space and $\chi$ is the electric susceptibility $\left(\chi=n^{2}-1\right.$ where $\mathrm{n}$ is the index of refraction), and a plot of $P$ versus $E$ is a straight line. In the extreme case of very high fields, we can expect that $P$ will become saturated, in other words, it simply cannot increase linearly indefinitely with $E$ (just as in the case of ferromagnetic materials, where the magnetic moment becomes saturated at fairly low values of $H$ ).

The standard approach for describing nonlinear optical phenomena involves expressing the dielectric polarization in terms of the applied electric field as follows [5]

$$
P=\varepsilon_{0}\left(\chi E+\chi_{2} E^{2}+\chi_{3} E^{3}+\cdots\right)
$$

The usual linear susceptibility, $\chi$, is much greater than the coefficients of the nonlinear terms $\chi_{2}, \chi_{3}$, and so on, and hence the latter contribute noticeably only at high-amplitude fields. The second order or $E^{2}$ nonlinear optical susceptibility term can only occur in crystals that are noncentrosymmetric, that is, crystals which have no inversion symmetry. However, the cubic susceptibility exists in all crystals, even isotropic materials. In general for anisotropic materials, the $\chi_{2}$ term in expression (2.2) is a third rank tensor. The most common notation for the measure of nonlinearity of a crystal is the nonlinear coefficient $d$, which is also a tensor and can be related to the susceptibility by $\mathrm{d}_{i j k}=2\left(\chi_{2}\right)_{i j k}$. The form of tensor $\vec{d}$ has to be consistent with the symmetry of the crystal. Thus no even harmonics can be produced by materials with a center of symmetry or an inversion center. When a NLO material is chosen for an application, the NLO coefficient and the transparency range are among the 
most important properties. In general, a large NLO coefficient is a desired characteristic of a device crystal.

The second-order susceptibility gives rise to several different nonlinear optical phenomena. If two intense monochromatic waves with frequencies $\omega_{1}$ and $\omega_{2}$ propagate through a crystal with a sufficient second-order nonlinear dielectric susceptibility coefficient, new light waves with frequencies $\omega_{1}+\omega_{2}=\omega_{3}$ (sum-frequency generation) or $\omega_{1}-\omega_{2}=\omega_{4}$ (difference-frequency generation) can arise. A special case occurs when $\omega_{1}=\omega_{2}$ and $\omega_{3}=$ $2 \omega_{1}$, namely second-harmonic generation. The opposite process, called optical parametric oscillation (OPO), is also possible when an incident light wave $\omega_{3}$ is divided into two new waves $\omega_{1}$ and $\omega_{2}\left(\omega_{3}=\omega_{1}+\omega_{2}\right)$.

Along with a material having a second-order susceptibility term, birefringence is important for utilizing crystals in nonlinear optical applications. Birefringence is the anisotropy of the refractive index. In an isotropic medium $c=(\mu \varepsilon)^{-1 / 2}$ and the phase velocity is independent of the direction of polarization. In an anisotropic medium the situation is different. The electric susceptibility becomes a tensor and the dielectric response of a material can be described by means of the electric permeability tensor defined by $\varepsilon_{i j}=\varepsilon_{0}\left(1+\chi_{i j}\right)$. A wave propagating along $\mathrm{z}$, having its electric field parallel to $x$, will induce only $P_{x}$ and will consequently have a phase velocity $c_{x}=\left(\mu \varepsilon_{11}\right)^{-1 / 2}$. If, on the other hand, the wave is polarized parallel to $y$, it will propagate with phase velocity $c_{y}=\left(\mu \varepsilon_{22}\right)^{-1 / 2}$. This situation represents one of the most important consequences of the dielectric anisotropy of crystals, i.e., the phenomenon of birefringence in which the phase velocity of an optical beam depends on the direction of polarization of its electric field vector. Thus, there is a difference between the refractive indices of ordinary and extraordinary beams resulting in the light beams traveling at different speeds in the crystal. Because of the birefringence of a material, critical phase matching can occur.

For example, in the case of second harmonic generation of light, a major difficulty in 
generating large amounts of light arises from the frequency dependence of the refractive index, that is, dispersion. At some initial point where the incident, or $\omega$-wave, generates the second harmonic, or $2 \omega$-wave, the two are coherent. As the $\omega$-wave propagates through the crystal, it continues to generate additional contributions of second harmonic light, which all combine totally constructively only if they maintain a proper phase relationship. Yet the $\omega$ wave travels at a phase velocity $\mathrm{v}_{\omega}$ (typically different from the phase velocity $\mathrm{v}_{2 \omega}$ of the $2 \omega$ -wave). Thus, the newly emitted second harmonic periodically falls out of phase with some of the previously generated $2 \omega$-wave. When the intensity of the second harmonic emerging from a plate of thickness $l$ is computed, it turns out to be [1]

$$
I_{2 \omega} \propto \frac{\sin ^{2}[\Delta k l / 2]}{(\Delta k l / 2)^{2}}
$$

This yields the result that $I_{2 \omega}$ has its maximum value when $\Delta k=0$. When $\Delta k=0$, $k^{2 \omega}=2 k^{\omega}$ and the process is phase-matched. The condition $\Delta k=0$ also translates into

$$
n_{\omega}=n_{2 \omega}
$$

or index matching. So the indices of refraction at the fundamental and second-harmonic frequencies must be equal. In normally dispersive materials, the index of the ordinary wave or the extraordinary wave along a given direction increases with $\omega$. This makes it impossible to satisfy (2.4) when both the $\omega$ and $2 \omega$ beams are of the same type-that is, when both are extraordinary or ordinary. However, under certain circumstances, condition (2.4) can be satisfied by making the two waves be of different types. For example, in a negative uniaxial crystal, there exists a certain propagation direction for which the fundamental beam is launched as an ordinary ray and the second-harmonic beam will be generated along the same direction as an extraordinary ray. Specifically, $n_{e}^{2 \omega}\left(\theta_{m}\right)=n_{o}^{\omega}$. 


\subsection{Applications of the nonlinear optical materials}

There are two classes of materials: uniaxial materials, for which $n_{x}=n_{y} \neq n_{z}$ and biaxial materials, for which $n_{x} \neq n_{y} \neq n_{z}$. The uniaxial crystals can further be classified as positive uniaxial $\left(n_{x}=n_{y}<n_{z}\right)$ and negative uniaxial $\left(n_{x}=n_{y}>n_{z}\right)$. KDP, KD*P, and $\mathrm{LiNbO}_{3}$, are negative uniaxial crystals, meaning $n_{o}>n_{e}$; whereas $\mathrm{LiTaO}_{3}$ is positive uniaxial, $n_{o}<n_{e}[1,3]$. In the anisotropic crystals there exists a special direction called the optic axis where there is no birefringence, that is $n_{e}=n_{o}$. Knowing the type of crystal is important information when phase-matching in a second-harmonic generator is desired. Another essential characteristic of the NLO materials used for SHG is the nonlinear optical coefficient $d_{\text {eff }}$. This coefficient is defined as [1]

$$
d_{\text {eff }}(2 \omega)=[\chi(2 \omega)][\chi(\omega)]^{2} \frac{b \varepsilon_{0}^{2}}{2 N^{2} e^{3}}
$$

and it has units of $\mathrm{m} \mathrm{V}^{-1}$. $d_{\text {eff }}(2 \omega)$ can be determined from the linear susceptibilities at the fundamental and second harmonic frequencies. Also, the conversion efficiency $\eta_{S H G}$ from $\omega$ to $2 \omega$ in SHG increases as the square of the NLO coefficient $d_{e f f}$. In anisotropic media, the polarization of the second harmonic frequency will depend on the direction of propagation of the fundamental wave and its state of polarization, so a SHG tensor $\vec{d}_{\text {eff }}$ must be used. The symmetry of $\vec{d}_{\text {eff }}$ is the same as the symmetry of the piezoelectric tensor and the same as the symmetry of the transpose of the linear electro-optic tensor $\vec{r}$ which is correlated to the symmetry of the crystals. There are 32 NLO coefficients tabulated by crystal symmetry classes. Typical magnitudes of the $d_{\text {eff }}$ coefficients are $10^{-12} \mathrm{~m} \mathrm{~V}^{-1}$. For example, $\mathrm{LiNbO}_{3}$ has a value for the coefficient at $1.15 \mu \mathrm{m}$ of $d_{33}=-49 \mathrm{pm} \mathrm{V}^{-1}$ and KDP has a coefficient at $1.06 \mu \mathrm{m} \mathrm{d}_{36}=0.51 \mathrm{pm} \mathrm{V}^{-1}$. These and other important properties of the NLO crystals in this study are summarized in Table 2.1. In general, large values of the NLO coefficients combined with good transparency in the range of use of the crystals are preferred for device 
applications.

Table 2.1. Selected optical properties of the NLO crystals in this study [1].

\begin{tabular}{|c|c|c|c|c|}
\hline & $\mathrm{LiNbO}_{3}$ & $\mathrm{LiTaO}_{3}$ & $\mathrm{KDP}$ & $\mathrm{KD} * \mathrm{P}$ \\
\hline $\begin{array}{c}\text { Transparency } \\
\text { range }(\mu \mathrm{m})\end{array}$ & $0.35-4.50$ & $0.4-5.0$ & $0.17-1.6$ & $0.20-2.1$ \\
\hline Electro-Optic & $r_{33}=30.8$ & $r_{33}=35.8$ & $\mathrm{r}_{41}=8.8$ & $\mathrm{r}_{41}=8.8$ \\
\hline coefficients & $r_{31}=8.6, r_{22}=3.4$ & $r_{13}=7, r_{22}=1$ & $\mathrm{r}_{63}=10.5$ & $\mathrm{r}_{63}=25.7$ \\
\hline$(\mathrm{pm} / \mathrm{V})$ at 633 & $r_{51}=28$ & $\mathrm{r}_{51}=20$ & & \\
\hline \multicolumn{5}{|l|}{$\mathrm{nm}$} \\
\hline Refractive & $\mathrm{n}_{\mathrm{e}}=2.200$ & $\mathrm{n}_{\mathrm{e}}=2.180$ & $\mathrm{n}_{\mathrm{e}}=1.467$ & $\mathrm{n}_{\mathrm{e}}=1.465$ \\
\hline indices at 633 & $\mathrm{n}_{\mathrm{o}}=2.286$ & $\mathrm{n}_{\mathrm{o}}=2.176$ & $\mathrm{n}_{\mathrm{o}}=1.507$ & $\mathrm{n}_{\mathrm{o}}=1.504$ \\
\hline \multicolumn{5}{|l|}{$\mathrm{nm}$} \\
\hline Coefficient for & $\mathrm{d}_{\mathrm{eff}}=5.7 \mathrm{pm} / \mathrm{V}$ & $\mathrm{d}_{\mathrm{eff}}=9 \mathrm{pm} / \mathrm{V}$ & $\mathrm{d}_{\mathrm{eff}}=0.51$ & $\mathrm{~d}_{\mathrm{eff}}=0.46$ \\
\hline \multirow[t]{2}{*}{ SHG } & at $1300 \mathrm{~nm}$ & at $532 \mathrm{~nm}$ & $\mathrm{pm} / \mathrm{V}$ & $\mathrm{pm} / \mathrm{V}$ \\
\hline & & & at $1064 \mathrm{~nm}$ & at $1064 \mathrm{~nm}$ \\
\hline Optical damage & $400 \mathrm{MW} / \mathrm{cm}^{2}$ & $>500 \mathrm{MW} / \mathrm{cm}^{2}$ & $500 \mathrm{MW} / \mathrm{cm}^{2}$ & $>100 \mathrm{MW} / \mathrm{cm}^{2}$ \\
\hline threshold & 14 ns pulses & $10 \mathrm{~ns}$ pulses & $20 \mathrm{~ns}$ pulses & $20 \mathrm{~ns}$ pulses \\
\hline at $1064 \mathrm{~nm}$ & & & & \\
\hline
\end{tabular}

Efficient frequency conversion nearly always requires the use of a high-power pulsed pump laser. Therefore, resistance to optical damage is of particular importance in the selection of materials for nonlinear applications. Damage can result from thermal heating, 
multiphoton absorption, self-focusing and dielectric breakdown, among other mechanisms. The intensity necessary to induce damage depends on the material, wavelength, pulse duration of the intense source, and whether the nonlinear material is being operated in a phase-matched arrangement.

An interesting phenomenon directly related to the optical damage process is called photorefractivity. This is an effect in which the local index of refraction is changed by the light field when the crystal is subjected to intense laser radiation. It was also found that these changes were maintained after the laser beam had been switched off, but could be erased by strong, uniform illumination. The photorefractive effect is sometimes also referred to as realtime holography since no development of any emulsion is necessary to store information by means of interference between a reference beam and an object beam [4]. Holographic storage was indeed one of the first applications of the "optical damage". This effect was first observed in lithium niobate [4]. $\mathrm{LiNbO}_{3}$ and $\mathrm{LiTaO}_{3}$ are among the most efficient crystals for generation of real-time holography using relatively low intensity levels (e.g., $1 \mathrm{~W} / \mathrm{cm}^{2}$ ). For the photorefractive phenomena to be significant, impurities such as $\mathrm{Fe}$ or other donors must be present in the crystal. It is the energy levels of electrons in these impurities that are near the middle of the band gap of the crystal that facilitate the inducing of the photorefractive effect. In pure, single crystals of $\mathrm{LiNbO}_{3}$ with stoichiometric composition, the photorefractive effect is not very large.

Other important phenomena that appear in NLO materials are the electro-optic and acousto-optic effects. The electro-optic (EO) effect represents in essence the variation of the refractive index with electric field. The linear EO, or the Pockels effect, is the first order variation with the applied electric field $[1,4]$. Certain crystals exhibit a linear electro-optic effect such that the birefringence occurs when it is placed in an electric field and a beam of light is passed through the sample in the direction of the field. Induced birefringence is proportional to the first power of the applied electric field: $\Delta n=n_{o}^{3} K_{P} E$ where $K_{P}$ is the 
Pockels coefficient, and $n_{o}$ is the index with no field applied. The quadratic EO or the Kerr effect represents the variation with the square of the applied electric field or $\Delta n=\lambda K E^{2}$. Both Pockels and Kerr effects can be used to construct very fast optical shutters $\left(10^{-10} \mathrm{sec}\right)$ by placing them between crossed polarizers.

All of the electro-optical properties and NLO characteristics of the crystals are drastically affected by the level of impurities present in the as grown materials or by the stoichiometry of the crystals. For example, in $\mathrm{LiNbO}_{3}$ it is known that the optical damage threshold in congruent crystals is an order of magnitude lower than in the stoichiometric ones. For this reason, the use of LN congruent crystals in applications requiring high power laser pulses is limited. In this case, one solution consists of doping LN crystals with $\mathrm{MgO}$ (especially $5 \%$ doping) to increase their resistance to damage. Another alternative is possible through the use of pure LN with stoichiometric composition. These aspects will be discussed in more detail in the next chapter. 


\section{CHAPTER 3}

\section{CRYSTAL STRUCTURE, GROWTH, AND POINT DEFECTS}

\subsection{Lithium niobate and lithium tantalate}

\subsubsection{Crystal structure}

Single crystals of lithium niobate and lithium tantalate are at the same time ferroelectric, piezoelectric, and pyroelectric. They also have high nonlinear optical and electro-optic coefficients and photorefractive sensitivity. A few of their basic physical properties are listed in Table 3.1.

Table 3.1. Selected physical properties of $\mathrm{LiNbO}_{3}$ and $\mathrm{LiTaO}_{3}$ crystals.

\begin{tabular}{|ccc|}
\hline Property & $\mathrm{LiNbO}_{3}$ & $\mathrm{LiTaO}_{3}$ \\
\hline Density $\left(\mathrm{g} / \mathrm{cm}^{3}\right)$ & 4.64 & 7.46 \\
\hline Melting point $\left({ }^{\circ} \mathrm{C}\right)$ & 1250 & 1650 \\
\hline $\begin{array}{c}\text { Curie temperature }\left({ }^{\circ} \mathrm{C}\right) \\
\text { Thermal conductivity } \\
\left(\mathrm{W} / \mathrm{m}^{\circ} \mathrm{C}\right)\end{array}$ & 1142 & $\sim 630$ \\
\hline Hygroscopic & no & 1.12 \\
\hline
\end{tabular}

Lithium niobate and lithium tantalate crystals have the same structure, which is related to that of the $\mathrm{ABO}_{3}$ perovskites. The space group is $R 3 c$. The lattice structure is rhombohedrally centered hexagonal with $a=5.148 \AA$ and $c=13.863 \AA$ for $\mathrm{LiNbO}_{3}$ and $a=$ 
$5.154 \AA$ and $c=13.781 \AA$ for $\mathrm{LiTaO}_{3}$. The number of formula units per unit cell is six for both crystals.

In Figure 3.1, I have illustrated a sequence of oxygen octahedra containing the cations along the $c$ axis. It is significant to envision the structure of these crystals as this sequence of distorted octahedra of oxygen ions along the polar $c$-axis. The cations $\mathrm{Li}, \mathrm{Nb}$, and $\mathrm{Ta}$ are located near the center of alternating octahedra with the repeating sequence $\mathrm{Li}, \mathrm{Nb} / \mathrm{Ta}$, and an empty octahedron.

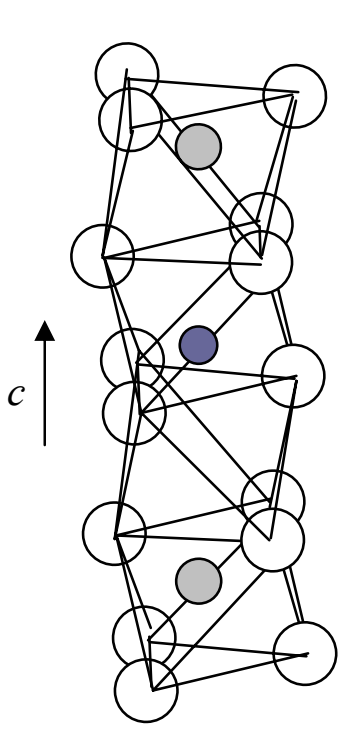

(a)
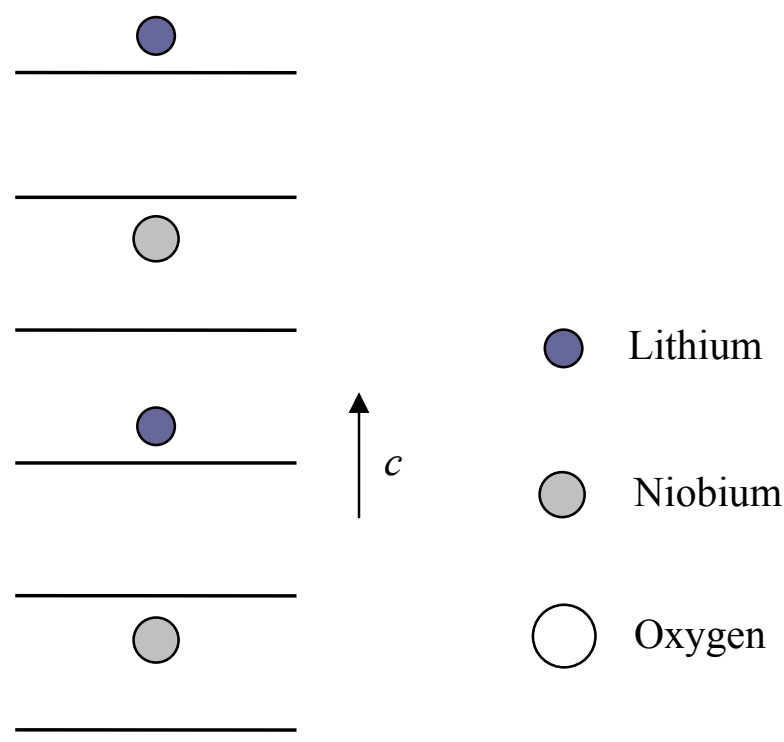

(b)

Figure 3.1. Model of $\mathrm{LiNbO}_{3}$ structure in the ferroelectric phase. (a) Sequence of distorted octahedra along the polar $c$-axis. (b) Side view of the vertical planes showing the vacant octahedra.

The oxygen layers are truly planar and are equidistant from each other. In the ferroelectric phase, the cations are actually displaced from their ideal positions midway between the 
oxygen layers. They are displaced away from each other and towards an empty octahedron in the same column. All of the $\mathrm{Nb}$ atoms are displaced in the one direction and all of the $\mathrm{Li}$ atoms are displaced in the opposite direction. These relative displacements of the $\mathrm{Nb}$ and $\mathrm{Li}$ ions are an important feature of the structure and a consequence of its polar symmetry. In single crystals grown from a congruent melt the ideal structure is distorted because of the large numbers of cation-related vacancies and impurities defects.

\subsubsection{Growth of $\mathrm{LiNbO}_{3}$ and $\mathrm{LiTaO}_{3}$}

Lithium niobate and lithium tantalate crystals are usually pulled from melts contained in platinum crucibles (i.e., the Czochralski method). The $\mathrm{LiNbO}_{3}$ starting materials are prepared by firing mixtures of $\mathrm{Li}_{2} \mathrm{CO}_{3}$ and $\mathrm{Nb}_{2} \mathrm{O}_{5}$ at $1100^{\circ} \mathrm{C}$. Similar procedures are employed for the growth of $\mathrm{LiTaO}_{3}$ and a more detailed description is given below. The primary problem encountered during the growth of $\mathrm{LiNbO}_{3}$ and $\mathrm{LiTaO}_{3}$ is determining the charge composition that leads to uniform crystals along the growth direction [10]. The stoichiometry of the crystals is defined by the ratio $[\mathrm{Li}] /[\mathrm{Nb}]$ and $[\mathrm{Li}] /[\mathrm{Ta}]$, respectively, in the crystals and is nearly always different from the ratio $[\mathrm{Li}] /[\mathrm{Nb}]$ or $[\mathrm{Li}] /[\mathrm{Ta}]$ in the melt. In a study by Cabanes et al. [9], $\mathrm{LiNbO}_{3}$ crystals were grown from a melt having the ratio of $[\mathrm{Li}] /[\mathrm{Nb}]=1$ and the resulting boule had a ratio of 0.957 . This was close to the ratio of 0.94 for congruent materials and far from the ratio of 0.99 for crystals near stoichiometry. To obtain a crystal with stoichiometric composition, a Li-rich melt with $[\mathrm{Li}] /[\mathrm{Nb}]>1$ is often used.

The $\mathrm{LiNbO}_{3}$ crystals used in the present investigation were cut from a large boule grown at Deltronic Crystal Industries (Dover, NJ). This boule was pulled from a lithium-rich melt that was doped with $1 \% \mathrm{MgO}$. The resulting composition was very nearly stoichiometric, i.e., the $[\mathrm{Li}] /[\mathrm{Nb}]$ ratio was approximately 0.99 or greater.

The $\mathrm{LiTaO}_{3}$ crystals used in this study were obtained from Yamaju Ceramics (Japan). As-received, these were congruently grown optical grade samples in the form of c plates. 
Several samples were given a post-growth vapor-transport-equilibration (VTE) treatment $[40,52]$ at Stanford University. In this procedure, the congruent $\mathrm{LiTaO}_{3}$ wafer is placed in a platinum crucible over a mixture of $\mathrm{Li}_{3} \mathrm{TaO}_{4}$ and $\mathrm{LiTaO}_{3}$ polycrystalline powder with a surrounding static atmosphere of dry $\mathrm{O}_{2}$ or $\mathrm{N}_{2}$. The crucible and sample are held near $1360^{\circ} \mathrm{C}$ for approximately 200 hours. Lithium ions diffuse into the crystal during this treatment and eliminate nearly all of the lithium vacancies and their corresponding tantalum antisite charge compensators $\left(\mathrm{Ta}_{\mathrm{Li}}\right)$ that had formed during the initial congruent growth. The resulting composition is very nearly stoichiometric after the VTE treatment, i.e., the [Li]/[Ta] ratio is greater than 0.99 . Stoichiometry is expected to influence the point-defect structure of the crystal and therefore may markedly influence many optical properties. In fact, ferroelectric Curie temperature, thermal expansion, absorption band edge, uv-excited luminescence, and $\mathrm{OH}$-vibrational bands in proton-doped material have been found to depend on the $[\mathrm{Li}] /[\mathrm{Nb}]$ ratio $[9]$.

\subsubsection{Point defects in $\mathrm{LiNbO}_{3}$ and $\mathrm{LiTaO}_{3}$}

An important characteristic of lithium niobate and lithium tantalate crystals that

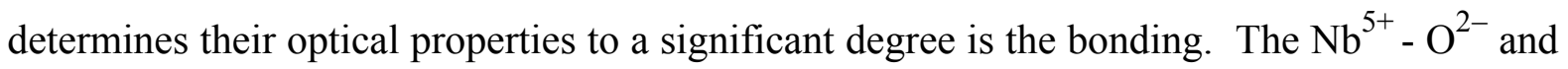
$\mathrm{Ta}^{5+}-\mathrm{O}^{2-}$ bonds are predominantly covalent and considerably stronger than the $\mathrm{Li}^{+}-\mathrm{O}^{2-}$ bond, which is purely ionic. The radii of the $\mathrm{Li}$ and $\mathrm{Nb}(\mathrm{Ta})$ ions are almost the same. These properties have the result that the composition of a $\mathrm{LiNbO}_{3}$ or $\mathrm{LiTaO}_{3}$ crystal can be appreciably deficient in lithium. Thus, these two crystals have a tendency to nonstoichiometry with $[\mathrm{Li}] /[\mathrm{Nb}]<1$ and $[\mathrm{Li}] /[\mathrm{Ta}]<1$. This tendency to non-stoichiometric compositions is the main cause for the existence of point defects in these two crystals. The variation from stoichiometry in lithium niobate and lithium tantalate crystals can be accommodated through a variety of defects, including cation vacancies, interstitials, and/or impurities. 
In a $\mathrm{LiNbO}_{3}$ or $\mathrm{LiTaO}_{3}$ crystal the main intrinsic defects are lithium vacancies, niobium (or tantalum) antisites, and oxygen vacancies. In stoichiometric crystals, the number of lithium vacancies and consequently the number of niobium (or tantalum) antisites are much smaller than in congruent crystals. There are two classes of point defects in $\mathrm{LiNbO}_{3}$ and $\mathrm{LiTaO}_{3}$ : (1) hole traps such as $\mathrm{Li}^{+}$vacancies and (2) electron traps such as $\mathrm{Nb}^{5+} / \mathrm{Ta}^{5+}$ ions, $\mathrm{Fe}^{3+}$ or $\mathrm{Cu}^{2+}$ ions on $\mathrm{Li}^{+}$sites, and oxygen vacancies. A Li-deficient composition means that $\mathrm{Nb}^{5+} / \mathrm{Ta}^{5+}$ ions occupy Li sites (and form antisite defects) to provide the necessary positive charge that keeps the crystal electrically neutral. In both congruent and stoichiometric $\mathrm{LiNbO}_{3}$ and $\mathrm{LiTaO}_{3}$ crystals, the presence of hydrogen is demonstrated by the existence of vibrational absorption bands of $\mathrm{OH}^{-}$molecular ions in the infrared region. The $\mathrm{OH}^{-}$absorption bands can be removed by annealing at $1000^{\circ} \mathrm{C}$ in a dry atmosphere and can be introduced by annealing in a wet atmosphere. Another family of point defects often observed in $\mathrm{LiNbO}_{3}$ and $\mathrm{LiTaO}_{3}$ are the transition metal ions. These impurities are either intentionally doped into the crystals or are unintentionally present in trace amounts. Transition metals are known for their strong influence on the optical absorption and on the photorefractive effect ("laser damage"). For example, Fe is introduced in LN and LT crystals utilized in optical storage devices because of the increased optical damage. Conversely, other impurities such as $\mathrm{Zn}$ and especially $\mathrm{Mg}$ decrease the optical damage, and crystals doped with these impurities are used in high power laser applications like SHG.

It is established that any change toward stoichiometry reduces the thermal and photoinduced effects associated with point defects. It is also established that any departure from stoichiometry leads to a high degree of structural disorder in the lithium niobate/tantalate crystals $[37,38,39,40]$. The congruently grown crystals fall in this last category. Such crystals therefore have a very high concentration of intrinsic defects. This also explains why $\mathrm{LiNbO}_{3}$ and $\mathrm{LiTaO}_{3}$ can tolerate high concentrations of extrinsic impurity ions. Possible charge misfits can easily be compensated by suitable intrinsic defects. 
Because of the similarity of the $\mathrm{Li}$ and $\mathrm{Nb} / \mathrm{Ta}$ sites, one of the most difficult questions to answer with respect to extrinsic defects in $\mathrm{LiNbO}_{3}$ is: are dopant ions substituting on the lithium site or on the niobium/tantalum sites? These remarks characterize the situation one is facing in the investigation of defects in $\mathrm{LiNbO}_{3}$ and $\mathrm{LiTaO}_{3}$.

In many of the earlier investigations, it was established that changes in the optical properties may be controlled by heat treatment under oxidation-reduction conditions [8]. A series of studies on the radiation-induced changes in the optical characteristics have been performed on congruently grown lithium niobate and lithium tantalate crystals, and also on stoichiometric crystals. Many authors [8,9] have observed changes in the optical absorption spectra of lithium niobate and lithium tantalate crystals after exposure to ionizing radiation or after annealing in a reducing atmosphere. On the basis of data from optical and EPR experiments, the structures of a number of intrinsic color centers, of both electron and hole nature, were determined.

A majority of the earlier defect-related studies in single crystals of lithium niobate and lithium tantalate have focused on the properties of transition-metal and rare-earth impurity ions. On one hand, $\mathrm{Fe}$ and $\mathrm{Cu}$ are considered donor impurities and lithium niobate crystals doped with these ions have increased $n$-type conduction and thus low optical damage resistance [41]. On the other hand, $\mathrm{Zn}^{2+}, \mathrm{In}^{3+}$, and $\mathrm{Mg}^{2+}$ are known as dopants which increase the damage resistance of $\mathrm{LN}$ by substituting for $\mathrm{Li}$ vacancies, thus acting as acceptors and decreasing the optical damage.

In a study by Sweeney et al. [12] it was shown that a threshold phenomenon takes place for stoichiometric $\mathrm{LiNbO}_{3}$ crystals doped with $5 \% \mathrm{MgO}$. This type of crystal has a smaller number of $\mathrm{Nb}$ antisites $\left(\mathrm{Nb}_{\mathrm{Li}}\right)$, fewer types of point defects created by irradiation or reduction treatments $\left(\mathrm{Nb}^{4+}\right.$ electron traps, $\mathrm{F}$, and $\mathrm{F}^{+}$-centers), and less thermally stable point defects as compared with the congruent LN. The same authors observed an emission band peaking near $430 \mathrm{~nm}$ in both $\mathrm{x}$-ray-induced luminescence and thermoluminescence 
experiments on $\mathrm{LiNbO}_{3}$ crystals grown with various combinations of $\mathrm{Mg}$-doping and stoichiometry. In this latter work, it was shown that incorporating $5 \% \mathrm{Mg}$ in a $\mathrm{LiNbO}_{3}$ crystal increased the intensity of its "blue" emission by nearly two orders of magnitude. This emission band was observed in an earlier investigation, by Krol et al. [10] who showed that an emission band peaking near $440 \mathrm{~nm}$ could be produced at low temperature in a lithiumrich powder of $\mathrm{LiNbO}_{3}$ using either an ultraviolet lamp (wavelengths less than $310 \mathrm{~nm}$ ) or a pulsed nitrogen laser $(337 \mathrm{~nm})$ for excitation. Arizmendi et al. [11], using x-ray excitation at $15 \mathrm{~K}$, produced a similar emission band peaking near $425 \mathrm{~nm}$ in congruently grown crystals of $\mathrm{LiNbO}_{3}$. Subsequent investigations $[9,13-15]$ verified that the luminescence in the 430 to $440 \mathrm{~nm}$ region is much more intense in stoichiometric and $\mathrm{Mg}$-doped $\mathrm{LiNbO}_{3}$ than in congruent $\mathrm{LiNbO}_{3}$. Many of the early investigators $[10,11,16]$ suggested that the electronhole recombination process responsible for the blue emission in $\mathrm{LiNbO}_{3}$ occurs at the regular unperturbed $\mathrm{NbO}_{6}$ units, in direct analogy with $\mathrm{CaWO}_{4}$ and related scintillator materials.

Several other investigations have described the infrared absorption characteristics of $\mathrm{OH}^{-}$molecular ions $[8,38,42,43] . \mathrm{OH}^{-}$stretching vibration bands at frequencies $3539 \mathrm{~cm}^{-1}$ and $3483 \mathrm{~cm}^{-1}$ with a half-width of about $30 \mathrm{~cm}^{-1}$ were reported in undoped and Mg-doped $\mathrm{LiNbO}_{3}$.

In the near infrared region a different type of absorption band was observed $[12,44]$. These authors have reported that after a vacuum anneal at high temperatures $\left(\sim 1000^{\circ} \mathrm{C}\right)$ $[12,44]$ a very broad band with the peak around $1200 \mathrm{~nm}$ is formed at low temperature in stoichiometric $\mathrm{Mg}$ or $\mathrm{Zn}$-doped (above the threshold) $\mathrm{LiNbO}_{3}$ and it cannot be formed in congruent LN crystals or in crystals doped with concentrations below the threshold. Correlation of the optical absorption data with EPR data representing a broad signal with $\mathrm{g}=$ 1.82, has lead to the conclusion that this absorption band is an electron trap center, most likely $\mathrm{Nb}^{4+}$ on a regular niobium site [12].

Other point defects detected by EPR measurements are $\mathrm{Fe}^{3+}$, niobium antisites 
$\left(\mathrm{Nb}_{\mathrm{Li}}{ }^{4+}\right), \mathrm{F}^{+}$-centers, and hole-traps. The $\mathrm{Nb}^{4+}$ center is a paramagnetic defect with $\mathrm{g}=1.90$ and the dominant feature in its EPR spectrum is a ten-line hyperfine pattern arising from the ${ }^{93} \mathrm{Nb}(I=9 / 2,100 \%$ abundant $)$ isotope. Schirmer and von der Linde [45] were the first to describe the $\mathrm{Nb}^{4+}$ center and they suggested that it was a self-trapped electron at a niobium ion occupying a normal site in the otherwise perfect lattice. Another possibility which was considered by Sweeney et al. [12] is that an antisite niobium $\mathrm{Nb}_{\mathrm{Li}}{ }^{4+}$ acts as the stabilizing entity for the electron. This can be explained by considering the nature of the bonds. The electron is significantly delocalized onto the surrounding oxygen ions of the $\mathrm{Nb}$ because of the strongly covalent nature of the niobium-oxygen molecular cluster and is more stabilized in the $\mathrm{Li}$ site for the $\mathrm{Li}$ - $\mathrm{O}$ bond is considered purely ionic.

A majority of the earlier defect-related studies in single crystals of $\mathrm{LiTaO}_{3}$ have focused on the properties of transition-metal and rare-earth impurity ions [17,27]. Also, several investigations have described the infrared absorption characteristics of $\mathrm{OH}^{-}$ molecular ions [28-30]. A series of reduction and radiation studies have been performed on congruently grown $\mathrm{LiTaO}_{3}$ crystals [31-36]. Heating a crystal to temperatures between 850 and $950^{\circ} \mathrm{C}$ in a reducing atmosphere produces a broad optical absorption band peaking near $460 \mathrm{~nm}$. Subsequent optical bleach at $77 \mathrm{~K}$ with an unfiltered xenon lamp converts this band to another band peaking near $579 \mathrm{~nm}$. The $460-\mathrm{nm}$ band recovers when the crystal is returned to room temperature following the low-temperature optical bleach. Furthermore, the 460-nm absorption band initially induced by the reduction treatment is removed if a reduced crystal is heated to $900^{\circ} \mathrm{C}$ in air (i.e., an oxidizing atmosphere).

An EPR signal representing an electron trapped at a $\mathrm{Ta}^{5+}$ ion (giving a $\mathrm{Ta}^{4+}$ ion with the $5 \mathrm{~d}^{1}$ configuration and $\mathrm{S}=1 / 2$ ) is produced during the $77-\mathrm{K}$ optical bleach of the reduced $\mathrm{LiTaO}_{3}$ crystals [32]. In separate experiments, exposing an as-grown congruent $\mathrm{LiTaO}_{3}$ crystal to ionizing radiation near $77 \mathrm{~K}$ produces the EPR-active Ta ions and a unique threeline EPR signal representing a trapped hole center. These radiation-induced signals 
thermally decay between 100 and $200 \mathrm{~K}$ [31].

\section{2. $\mathrm{KH}_{2} \mathrm{PO}_{4}$ and $\mathrm{KD}_{2} \mathrm{PO}_{4}$}

\subsubsection{Crystal structure}

In the past several decades, the physical properties of KDP and DKDP have been studied extensively. The two crystals have similar structure and similar physical properties. A few of their optical properties are described in Table 2.1. In Table 3.2 other physical properties are given for the two crystals. There are not many differences between these materials in the properties listed below (the primary difference is in the Curie temperature).

Table 3.2. Selected physical properties of KDP and KD*P crystals.

\begin{tabular}{|ccc|}
\hline Property & KDP & $K D^{*} P$ \\
\hline Density $\left(\mathrm{g} / \mathrm{cm}^{3}\right)$ & 2.3325 & 2.3555 \\
\hline Melting point $\left({ }^{\circ} \mathrm{C}\right)$ & 523 & 523 \\
\hline Curie temperature $\left({ }^{\circ} \mathrm{C}\right)$ & 123 & 222 \\
\hline $\begin{array}{c}\text { Thermal conductivity } \\
\left(\mathrm{W} / \mathrm{m}^{\circ} \mathrm{C}\right)\end{array}$ & 19 & 19 \\
\hline Hygroscopic & yes & yes \\
\hline
\end{tabular}

In what follows, for simplicity, I will describe the KDP crystalline structure. The crystalline structure of the isomorphic $\mathrm{KD} * \mathrm{P}$ is the same with the exception that deuterium replaces hydrogen. At room temperature, $\mathrm{KH}_{2} \mathrm{PO}_{4}$ has a noncentrosymmetric tetragonal (ferroelectric) lattice (point group I42d) with lattice constants $a=7.448 \AA$ and $c=6.977 \AA$. 
There are 32 atoms in the primitive unit cell of KDP, and each unit cell is formed by four formula units. The KDP lattice is composed of two sets of $\mathrm{PO}_{4}$ groups linked to each other by hydrogen bonds. These $\mathrm{PO}_{4}$ groups are rotated $16^{\circ}$ about the $c$ axis, off the $a$ axes. There are also two distinct potassium ion positions. In particular, each phosphorus ion is surrounded by four oxygen ions located at the vertices of a nearly regular tetrahedron (contracted along the $\mathrm{c}$ axis by approximately $2 \%$ ). Each $\mathrm{PO}_{4}$ group is linked to four other $\mathrm{PO}_{4}$ groups, spaced $\mathrm{c} / 4$ apart along the $\mathrm{c}$ axis, by hydrogen bonds. Figure 3.2 represents a projection of the tetragonal KDP lattice onto the $\mathrm{c}$ plane. The $\mathrm{PO}_{4}$ groups and the potassium ions are arranged in such a manner

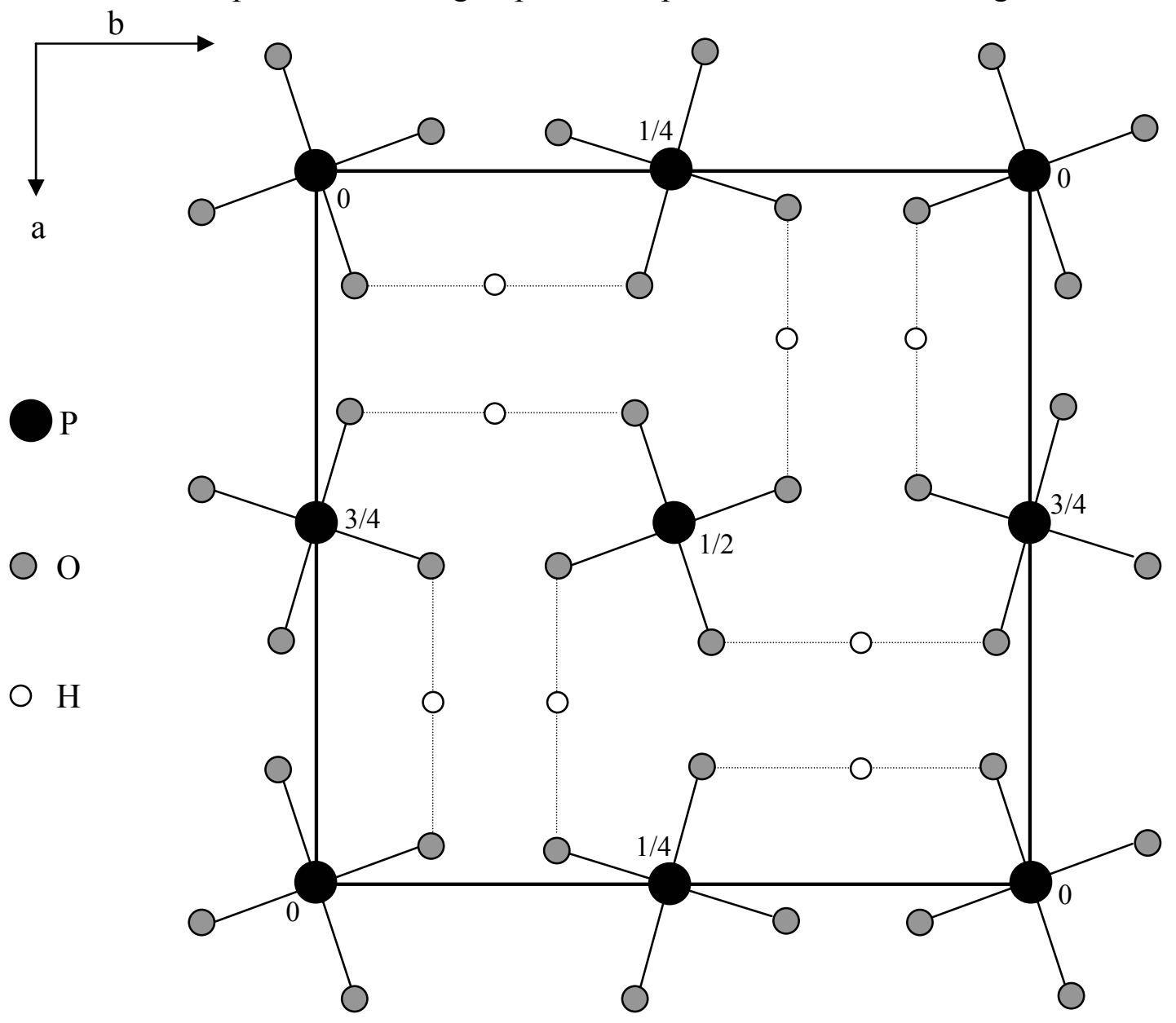

Figure 3.2. Room temperature tetragonal structure of KDP. All atoms contained in one cell are illustrated by the solid lines. Phosphorus ions lie at the height marked in the figure. 
that potassium and phosphorus ions are spaced at a distance of $\mathrm{c} / 2$ along the $\mathrm{c}$ axis. The $\mathrm{PO}_{4}$ tetrahedra are also connected by potassium ions. Each potassium ion is surrounded by eight oxygen ions with four of these oxygens belonging to tetrahedra of neighboring columns. One set of four oxygen ions lies closer to the potassium ion than the other set of four oxygen ions. The K-O bond lengths are $2.89 \AA$ and $2.82 \AA$, respectively. A hydrogen bond involves one upper and one lower oxygen atom of the neighboring $\mathrm{PO}_{4}$ units. As a result, all hydrogen bonds lie in a plane nearly perpendicular to the c axis of the crystal.

The heavy solid lines in Figure 3.2 indicate the unit cell. There are 16 oxygen ions and eight hydrogen ions contained within these solid lines. And there is one phosphorus ion contained within the solid lines and three additional phosphorus ions on the solid lines which are part of the unit cell. The four potassium ions in the unit cell are not shown. These potassium ions lie above and below the phosphorus ions. In this figure, the dotted lines show the hydrogen bonds. The upper oxygens of one tetrahedron lie at the same level as the lower oxygens of two neighboring tetrahedra, and the O-O distances are short, about $2.49 \AA$. These are obvious positions for the hydrogen bond. The hydrogen ions link the tetrahedra into a three-dimensional framework.

As the temperature is decreased below $123 \mathrm{~K}$, the KDP lattice undergoes a first-order ferroelectric phase transition and the structure changes from tetragonal to orthorhombic. The crystal structure of the orthorhombic phase has space group Fdd2 and lattice parameters are $a$ $=10.54 \AA, b=10.46 \AA$, and $c=6.92 \AA$, with $c$ being the ferroelectric axis. In this phase, the $\mathrm{PO}_{4}$ units are distorted as a result of the phosphorus ion moving slightly along the $c$ axis relative to its four neighboring oxygens, i.e., away from two of the oxygen ions and toward the other two oxygen ions. Two types of structural domains exist in the ferroelectric phase of the crystal. In one type, each $\mathrm{PO}_{4}$ unit has both its associated hydrogen atoms near the upper end of the tetrahedron, whereas in the other type, the $\mathrm{PO}_{4}$ group has both hydrogens near the lower end of the tetrahedron. These two different structures give rise to domains of 
spontaneous electric polarization along the $+c$ and $-c$ that are characteristic of the ferroelectric phase. It has been shown experimentally that the positive end of the dipole is that towards which the $\mathrm{P}$ atom is displaced (i.e., downwards in Figure 3.3 (i) and upwards in (ii)).

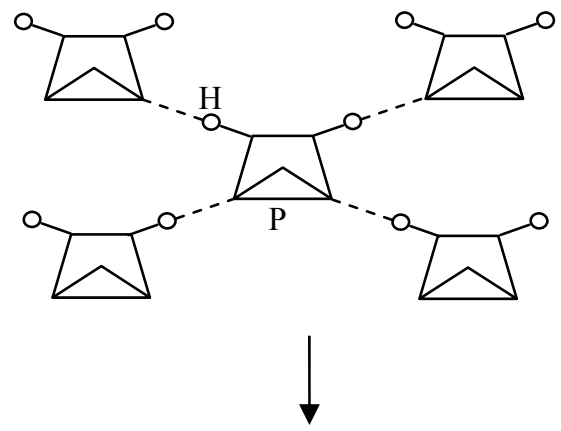

(i)

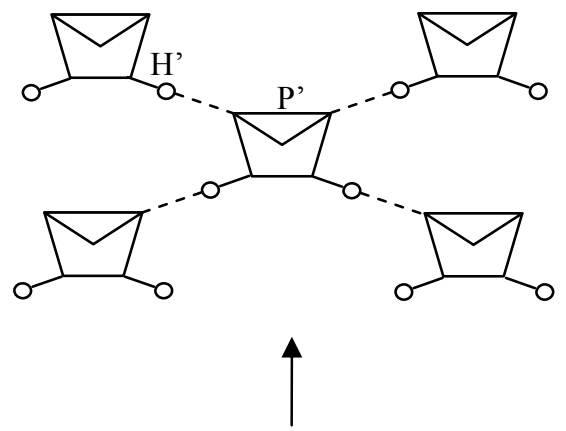

(ii)

Figure 3.3. Schematic diagram of ferroelectric reversal. The tetrahedra are shown in outline, with their short $\mathrm{P}-\mathrm{O}$ bonds inserted. The $\mathrm{H}$ atoms are represented by small circles, the hydrogen bonds by broken lines. The direction of the dipole is shown by the arrow. During reversal, from (i) to (ii), P moves to P', H to H'; and oxygens while moving very little, interchange their environments.

This is the direction away from the $\mathrm{H}$ atoms. The electron clouds of the oxygen atoms are distorted by the close approach of $\mathrm{P}$, and the charges are redistributed accordingly. A similar result was found in $\mathrm{LiNbO}_{3}$, where the dipoles are $\mathrm{NbO}_{6}$ octahedra; the positive end is that towards which the $\mathrm{Nb}$ was displaced. It is the relative displacement of the phosphorus and the group of oxygens that makes the dipole; the role of the hydrogen is in its effect on the polarization of the oxygens, in which it cooperates with the central cation $\mathrm{P}$.

A polar structure is of interest as a ferroelectric if its dipoles are reversible and this is obviously easy in KDP. This can happen when the P moves within the tetrahedron and the $\mathrm{H}$ moves within the hydrogen bond, as it is shown in Figure 3.3. The dipole direction is 
indicated by the arrow. In (i) it points downward. When a field pointing upward is applied, $\mathrm{P}$ moves upward, and in doing so it repels the $\mathrm{H}$ atoms at the upper corners, which move out along the hydrogen bond and become attached to the lower corners of adjacent tetrahedra, made available by the upward movement of $\mathrm{P}$ atoms in these tetrahedra. We can see that the result, propagated from one tetrahedron to the next, is a reversal of direction of the whole structure, as shown in Figure 3.3 (ii). A more detailed discussion of the orthorhombic phase of KDP is found in Reference 6.

\subsubsection{Growth of $\mathrm{KH}_{2} \mathrm{PO}_{4}$ and $\mathrm{KD}_{2} \mathrm{PO}_{4}$}

Single crystals of KDP and KD*P can be grown from solution. In a typical approach, crystals are grown only in the [001] direction by slow growth of the $\{101\}$ faces. To obtain reasonable sized crystals employing the conventional method, growth should start from a seed plate that has the same cross-section dimensions as the final crystal; this, however, presents a problem because obtaining the seeds of the required size is difficult. In the past decades, the growth technique has developed greatly. Large aperture crystals can be grown by various methods, such as temperature decreasing, solution flowing, and the recently developed rapid growth method. The rapid-growth method uses a point seed, high supersaturation, and extensive purification of the raw material. The final size of the crystals does not depend on the initial size of the seed, which is typically about $1 \mathrm{~cm}^{3} . \mathrm{KD} * \mathrm{P}$ crystals can be grown from solution with different deuterium fraction.

\subsubsection{Point defects in $\mathrm{KH}_{2} \mathrm{PO}_{4}$ and $\mathrm{KD}_{2} \mathrm{PO}_{4}$}

In the application of KDP and $\mathrm{KD} * \mathrm{P}$ for the third and fourth harmonic generation of neodymium-based near-infrared solid-state lasers, an intense pump beam is employed and laser-induced optical absorption may appear in the bulk of these materials leading to 
limitations in device performance [55-57]. Impurities and vacancies, introduced during crystal growth, are expected to play important roles in this optical damage process.

In this section we will describe some of the most important point defects in potassium dihydrogen phosphate (they also occur in the deuterated crystals). The focus of this discussion will be on the defects which are well studied and for which the models characterizing them are well understood. Also, those defects with direct implication on the optical properties of the two crystals will be given more attention. The best studied intrinsic defects in KDP and KD*P crystals, which were reliably identified by EPR, are the $A$ radical $\left(\mathrm{HPO}_{4}\right)^{-}$, the $B$ radical $\left(\mathrm{H}_{2} \mathrm{PO}_{4}\right)^{0}$, the interstitial hydrogen atom $\mathrm{H}^{0}$, the silicon impurity located on a phosphorus ion site, and the $\left(\mathrm{PO}_{3}\right)^{2-}$ molecular ions. I will describe each separately.

The $A$ radical $\left(\mathrm{HPO}_{4}\right)^{-}$center was the first hole center to be reported, and subsequent studies have shown that it consists of a hole trapped on a single oxygen ion adjacent to a hydrogen vacancy [58-62]. The regular $\mathrm{KH}_{2} \mathrm{PO}_{4}$ lattice contains $\mathrm{K}^{+}$ions and $\left(\mathrm{H}_{2} \mathrm{PO}_{4}\right)^{-}$units. Upon removal of a proton, an $\left(\mathrm{H}_{2} \mathrm{PO}_{4}\right)^{-}$unit becomes $\left(\mathrm{HPO}_{4}\right)^{2-}$ which then easily stabilizes a hole and forms a paramagnetic $\left(\mathrm{HPO}_{4}\right)^{-}$. The analog in $\mathrm{KD}_{2} \mathrm{PO}_{4}$ is the $\left(\mathrm{DPO}_{4}\right)^{-}$hole center. These point defects can be created when intense ultraviolet light propagates through the crystals. The EPR spectrum of this defect consists of a pair of lines arising from a phosphorus hyperfine interaction $\left({ }^{31} \mathrm{P}\right.$ is $100 \%$ abundant with $\left.I=1 / 2\right)$. The effective $g$ value is 2.0217 when the magnetic field is parallel to the $c$ axis [62].

A separate defect created under similar conditions as the proton vacancy hole center (i.e., exposure at low temperature to ionizing radiation or a high-power $266 \mathrm{~nm}$ laser beam), was observed using EPR and ENDOR techniques [60,62]. This defect, consisting of a pair of three EPR lines in KDP, was labeled $\left(\mathrm{H}_{2} \mathrm{PO}_{4}\right)^{0}$ or radical $B$. This is the self-trapped hole center. The observed EPR spectrum can be interpreted as a hyperfine interaction with one phosphorus and two equivalent protons. In addition to the characteristic $31 \mathrm{G}$ phosphorus 
hyperfine slitting, the $\left(\mathrm{H}_{2} \mathrm{PO}_{4}\right)^{0}$ center also has a resolved hyperfine pattern (a triplet with 3.2 G). The existence of such a defect in low-temperature irradiated $\mathrm{KH}_{2} \mathrm{PO}_{4}$ had been postulated by earlier investigators $[59,63,64]$, but Wells, Budzinski, and Box [60] were the first to identify its structure. Until the investigation by Stevens et al. in 1999 [62], it was believed that the $\left(\mathrm{H}_{2} \mathrm{PO}_{4}\right)^{0}$ centers could only be observed if the crystal was irradiated below $77 \mathrm{~K}$ (e.g., Wells, Budzinski, and Box [60] irradiated at $4.2 \mathrm{~K}$ ). If the $\mathrm{KH}_{2} \mathrm{PO}_{4}$ crystal is irradiated at a temperature slightly above $77 \mathrm{~K}$ or if tens of minutes elapse before the irradiated sample is cooled below $77 \mathrm{~K}$, most of the $\left(\mathrm{H}_{2} \mathrm{PO}_{4}\right)^{0}$ centers thermally decay and thus are not available for study [62].

In $\mathrm{KD}_{2} \mathrm{PO}_{4}$ crystals $\mathrm{x}$-irradiated at $77 \mathrm{~K}$ (266 nm gives similar results), the EPR and ENDOR measurements revealed the presence of $\left(\mathrm{D}_{2} \mathrm{PO}_{4}\right)^{0}$ centers and to a lesser extent of $\left(\mathrm{HDPO}_{4}\right)^{0}$ centers. The latter centers are present because the crystals are not $100 \%$ deuterated [62]. The EPR spectrum of a self-trapped hole center in KD*P consists of a pair of lines at approximately the same field position as the $\left(\mathrm{H}_{2} \mathrm{PO}_{4}\right)^{0}$, but they lack a resolved hyperfine structure. This absence of resolved hyperfine structure for the $\left(\mathrm{D}_{2} \mathrm{PO}_{4}\right)^{0}$ and $\left(\mathrm{HDPO}_{4}\right)^{0}$ centers is a result of the deuteron's smaller magnetic moment. Nevertheless, the nuclear quadrupole interaction with the deuteron $(I=1)$ appears in the ENDOR data as four lines instead of two from the nuclear interaction with the proton [62]. The hyperfine splitting of approximately $30 \mathrm{G}$ is due to phosphorus. The model that best represents the experimental data is a hole shared by two oxygen ions on one $\mathrm{PO}_{4}$ unit in the otherwise perfect lattice, having nuclear interaction with the phosphorus and the two near by bonded deuterium ions.

An interesting phenomena is that a broad transient optical absorption band covering much of the visible and near-uv region appears in KDP and $\mathrm{KD} * \mathrm{P}$ crystals when they are exposed at room temperature to intense $\left(\mathrm{GW} / \mathrm{cm}^{2}\right) 266 \mathrm{~nm}$ (below the band gap energy) laser pulses [55,56]. This phenomenon occurs when a two-photon absorption event (the combined energy of two $266 \mathrm{~nm}$ photons exceeds the band gap energy) produces a "free" electron and 
hole, which may then be trapped in the form of an interstitial hydrogen atom (or deuterium atom) and a localized hole center on a $\mathrm{PO}_{4}$ unit [61]. The resulting hole center is the cause of the visible and near-uv absorption. At room temperature, these laser-induced defects thermally decay in times on the order of milliseconds. However, if the crystals are irradiated at low temperatures $(<77 \mathrm{~K})$ then the hole center $A$ is stable up to the Curie temperature ( $123 \mathrm{~K}$ in $\mathrm{KH}_{2} \mathrm{PO}_{4}$ and $222 \mathrm{~K}$ in $\mathrm{KD}_{2} \mathrm{PO}_{4}$ ).

Dieguez et al. [63,64] in 1981 and 1984 studied in more detail the effect of $x$ ray irradiation on the optical absorption and luminescence spectra of KDP and KD*P. In their experiments, $\sigma$-polarized absorption bands at 390 and $550 \mathrm{~nm}$ were induced by low temperature $(10 \mathrm{~K}) \mathrm{x}$ ray irradiation. Also, a $\pi$-polarized band was observed peaking at $510 \mathrm{~nm}$ and $550 \mathrm{~nm}$ in $\mathrm{KH}_{2} \mathrm{PO}_{4}$ and $\mathrm{KD}_{2} \mathrm{PO}_{4}$, respectively. Based on correlations of the optical absorption data from their studies and EPR data from studies done by different authors $[59,65]$, they suggested that the absorption bands at 390 and $550 \mathrm{~nm}$ have an intrinsic character and that they arise from the self-trapped hole center, radical $B\left(\mathrm{H}_{2} \mathrm{PO}_{4}{ }^{0}\right.$ in $\mathrm{KDP}$ or $\mathrm{D}_{2} \mathrm{PO}_{4}{ }^{0}$ in $\left.\mathrm{KD} * \mathrm{P}\right)$. From thermal annealing measurements of the $\pi$-polarized band and thermoluminescence experiments the authors have attributed the $\pi$ band to the proton vacancy hole center. They have also observed that the efficiency of the radiation-induced production of radicals $A$ have decreased in $\mathrm{KD}^{*} \mathrm{P}$ as a result of deuterium substituting for hydrogen and explained that this may be possible since the breaking of the $\mathrm{O}-\mathrm{H}$ bond may be favored by the higher vibrational frequency of the proton.

A third intrinsic defect of significant interest in KDP is the interstitial hydrogen atom, a trapped electron center identified by EPR [60,61]. This center has a characteristic $502 \mathrm{G}$ proton hyperfine splitting and it can be formed in defect-free regions of the lattice [61]. Thermal stability is an important property of these "native" defects; the proton vacancy hole centers and the hydrogen atoms become unstable between 80 and $200 \mathrm{~K}$, while the selftrapped hole centers become unstable near $70 \mathrm{~K}$. Because they are not stable at room 
temperature, these three centers will appear only in a transient form during harmonic generation applications of KDP.

In addition to these intrinsic defects, Garces et al. in 2001 [66], using EPR, investigated a new hole trap and several new electron traps in undoped KDP. These centers are produced by ionizing radiation and they are thermally stable near room temperature. The EPR spectrum of the hole center consists of a single doublet (the width of each line is $2.86 \mathrm{G}$ and the splitting is $7.1 \mathrm{G}$ ) when the magnetic field is parallel to the $c$ axis. The suggested model is a hole trapped on an oxygen ion adjacent to a silicon impurity substituting for a phosphorus ion $\left(\mathrm{H}_{2} \mathrm{SiO}_{4}\right)^{-}$. The reasons for this assignment are as follows. First, there is a small, but significant, positive shift of the $g$ matrix strongly indicating that this is a holelike center and second, the $7.1 \mathrm{G}$ resolved $c$-axis hyperfine agrees well with the hydrogen hyperfine splitting for $\mathrm{H}_{2} \mathrm{PO}_{4}{ }^{0}$ centers. The hyperfine lines due to interaction with the $\mathrm{Si}$ nucleus are very weak $\left({ }^{29} \mathrm{Si} 4.7 \%\right.$ abundant, $\left.I=1 / 2\right)$. The same authors have also observed the EPR spectra from five $\left(\mathrm{PO}_{3}\right)^{2-}$ electron trap centers in KDP after first irradiating the KDP crystal at $77 \mathrm{~K}$ with $\mathrm{x}$ rays. The data were taken at room temperature. When the magnetic field is parallel to the $c$ axis, each of the five spectra consists of a widely split doublet (521 to $802 \mathrm{G})$ due to a phosphorus hyperfine interaction. The proposed model for the formation of the $\left(\mathrm{PO}_{3}\right)^{2-}$ electron centers is that during crystal growth, a regular $\left(\mathrm{PO}_{4}\right)^{3-}$ unit is replaced by a $\left(\mathrm{PO}_{3}\right)^{-}$unit (i.e., the unit contains an oxygen vacancy) and it traps an electron during exposure to ionizing radiation. The observation of five distinct $\left(\mathrm{PO}_{3}\right)^{2-}$ centers suggests that a cation vacancy is associated with each oxygen vacancy, i.e., either a proton or a potassium is missing from one of five inequivalent cation sites near the vacancy.

Besides the intrinsic defects and the silicon impurity discussed above which are believed to be present in all the KDP and KD*P crystals, there are other point defects like the transition metal ions impurities that can affect the optical properties of these crystals. In general, unless intentionally doped, most $\mathrm{KDP}$ and $\mathrm{KD} * \mathrm{P}$ crystals contain very small 
concentrations of transition-metal ions. Thus, electron paramagnetic resonance (EPR) spectra from impurities such as $\mathrm{Fe}^{3+}$ and $\mathrm{Cr}^{3+}$ are usually not detected in high-quality material. In such high quality, nominally pure $\mathrm{KDP}$ and $\mathrm{KD} * \mathrm{P}$ crystals, there is little extrinsic optical absorption present in the near ultraviolet region. However, doping KDP crystals with $5 \mathrm{ppm}$ of Fe introduces a broad charge-transfer absorption band peaking near $270 \mathrm{~nm}$. The tail of this band extends to short wavelengths and there is an additional peak near $210 \mathrm{~nm}$. This $270-\mathrm{nm}$ absorption band presents a problem for KDP crystals used as harmonic generators since it overlaps the fourth harmonic output $(266 \mathrm{~nm})$ of commonly used Nd:YAG lasers. Various investigators have suggested that transition metal ions such as $\mathrm{Fe}^{3+}$ may be responsible for the $270 \mathrm{~nm}$ absorption band in KDP $[67,68]$ even though a direct correlation between the two has never been proven.

In 2001, Garces et al. [69] published an experiment done on a KDP sample, to explain the origin of the $270 \mathrm{~nm}$ band by correlating EPR and optical absorption measurements. The experiment consists of an $\mathrm{x}$ ray irradiation at room temperature followed by a series of annealing treatments which show that while the EPR signal from $\mathrm{Fe}^{3+}$ decreases with the increase of annealing temperature, the $270 \mathrm{~nm}$ absorption band increases. This proves that there is no connection between the $\mathrm{Fe}^{3+}$ impurities and the $270 \mathrm{~nm}$ band. This result is interpreted by the authors as follows: some of the Fe impurities enter the KDP lattice, not as $\mathrm{Fe}^{3+}$, but instead as $\left(\mathrm{FeO}_{4}\right)^{2-}$ molecular units substituting for $\left(\mathrm{PO}_{4}\right)^{3-}$ with a nearby cation vacancy (either a hydrogen or a potassium) and then neutral complexes are readily formed in the as-grown crystals. 


\section{CHAPTER 4}

\section{EXPERIMENTAL TECHNIQUES AND INSTRUMENTATION}

\subsection{General description}

The experimental techniques used in the present research include thermoluminescence (TL), optical absorption in the ultraviolet, visible, and infrared regions of the spectrum, and electron paramagnetic resonance (EPR). All of these techniques can provide valuable information about point defects in crystals.

In general, investigations which attempt to describe the defect state of a material by using thermoluminescence alone are of limited value. This is because the TL data do not directly identify the participants in the recombination process. The most progress in recent years has been made when several different methods (e.g., optical absorption, photoconductivity, ionic conductivity, electron paramagnetic resonance) have been used to complement the thermoluminescence experiments [2]. Nevertheless, although it is less informative to use thermoluminescence alone to describe the defect structure of a solid, it is a very useful technique when combined with other measurements and it is very rare that thermoluminescence provides no information at all.

Electron paramagnetic resonance is a powerful technique widely used in a variety of disciplines and for a multitude of research problems. In some cases, EPR can provide enough information to fully describe the properties of a defect in a crystal. In this study, the experimental results from EPR provide additional information to help understand the results from the TL and optical absorption experiments.

Optical absorption spectroscopy is a commonly used technique to determine 
transparency range, absorption band edge, and absorption bands due to impurities or other defects that might be present in a material. Like EPR, the optical absorption experiments in this investigation helped to understand the TL data from $\mathrm{LiNbO}_{3}$ and $\mathrm{LiTaO}_{3}$. Also, optical absorption data were obtained from the DKDP crystals.

\subsection{Thermoluminescence}

Thermoluminescence (TL), or thermally stimulated luminescence (TSL), is the emission of light from an insulator or semiconductor when it is heated (after having been irradiated at a lower temperature). This is not to be confused with the light spontaneously emitted from a substance when it is heated to incandescence. More specifically, thermoluminescence is the thermally stimulated emission of light following the previous absorption of energy from radiation.

As an experimental technique, thermoluminescence finds favor in diverse scientific disciplines such as archaeology, geology, medicine, solid-state physics, biology, organic chemistry, and others. In principle, thermoluminescence experiments can be expected to yield useful information on the properties of the various point defects present within an insulator or semiconductor. As a means of detecting the presence of low concentrations of point defect, the sensitivity of thermoluminescence is very good. It is estimated that the technique is capable of detecting as few as $10^{9}$ defects in a specimen.

The fundamental principles which govern the production of thermoluminescence are essentially the same as those which govern all luminescence processes. However, in terms of a characteristic time from the absorption of the radiation until the emission of light, there are differences between luminescence (fluorescence or phosphorescence) and thermoluminescence. The emission of light takes place in a characteristic time $\tau_{\mathrm{c}}$ after the absorption of the radiation and this parameter allows classifying the luminescence 
process. In Figure 4.1, the luminescence is depicted as taking place nearly simultaneously (characteristic time, $\tau_{\mathrm{c}} \leq 10^{-8} \mathrm{~s}$ for fluorescence and $10^{-8} \leq \tau_{\mathrm{c}} \leq 10^{-4} \mathrm{~s}$ for phosphorescence) with the absorption of radiation, and stopping very quickly after the radiation ceases. For thermoluminescence, the characteristic time will depend on the sample temperature, and if the material is not warmed the thermoluminescence will not occur. In this figure, $T_{0}$ is the temperature at which the irradiation takes place and $\beta$ is the heating rate.

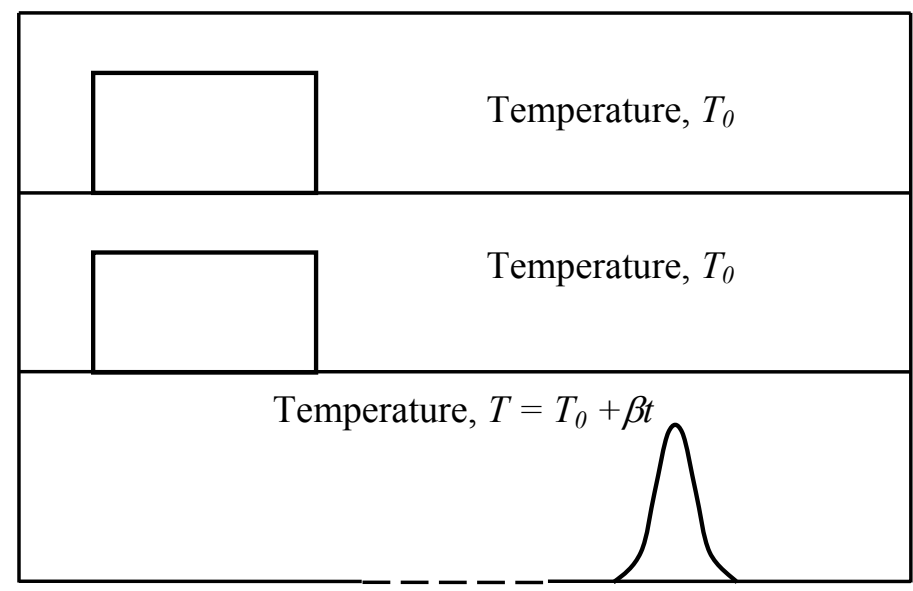

Intensity of exciting radiation

Luminescence intensity

Thermoluminescence intensity

Figure 4.1. Relationships between the absorption of radiation and the emission of luminescence and thermoluminescence.

In general, luminescence emission is explained by the transfer of energy from the incident radiation to the electrons of the solid, thus exciting the electrons from a ground state $g$ to an excited state e (transition (i) in Figure 4.2(a)). The emission of a photon takes place when an excited electron returns to its ground state (transition (ii)). Thus, for fluorescence, the delay between transitions (i) and (ii) is less than $10^{-8} \mathrm{sec}$ and this process is temperature independent. In 1935, Jablonski gave the first explanation of 
temperature dependent phosphorescence. Here (Figure 4.2(b)) the energy level diagram is modified by the presence of a metastable level $\mathrm{m}$ in the "forbidden" energy gap between e and $\mathrm{g}$. An electron excited from $\mathrm{g}$ to e can now become trapped at $\mathrm{m}$ where it will remain until it is given enough energy $E$ to return to e from where it can undergo a normal transition back to $\mathrm{g}$, with the subsequent emission of light. The delay observed in

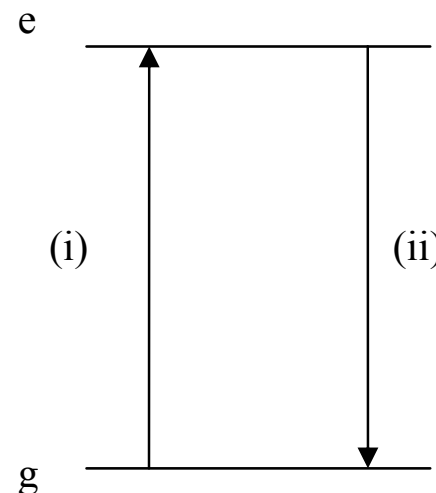

(a)

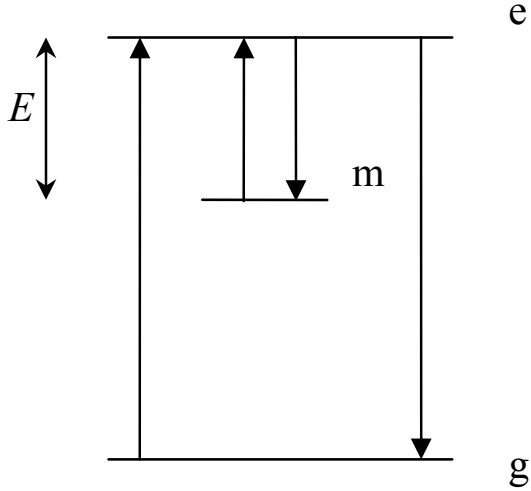

(b)

Figure 4.2. Energy transitions involved in the production of $(a)$ temperature independent luminescence and $(b)$ temperature dependent emission of light.

phosphorescence corresponds to the time the electron spends in the electron trap $\mathrm{m}$. From thermodynamic arguments, the mean time spent in the trap at temperature $T$ is given by

$$
\tau=s^{-1} \exp (E / k T)
$$

where $s$ is a constant, called the "frequency factor" or "attempt-to-escape frequency", $E$ is the energy difference between $\mathrm{m}$ and e (called the trap depth), and $k$ is Boltzmann's constant.

To explain thermoluminescence, a two-level simple model was developed by several authors [2] (Garlick and Wilkins in 1945; Randall and Wilkins in 1945; Garlick 
and Gibson in 1948). This simple model is shown in Figure 4.3. In this energy band scheme there are just two localized levels. One level acts as a trap center (T) and the other acts as a recombination center (R). In Figure 4.3, the trap is situated above the equilibrium Fermi level $E_{f}$ and thus is empty before the absorption of radiation. It is therefore a potential electron trap. The recombination center is situated below the Fermi level and is thus full of electrons and is a potential hole trap. The absorption of radiation of energy $(h v)_{a}>\left(E_{C}-E_{V}\right)$ (i.e., greater than the band-gap energy) results in the ionization of valence electrons, producing free electrons in the conduction band and free holes in the valence band (transition 1). The free carriers may either recombine with each other or become trapped.

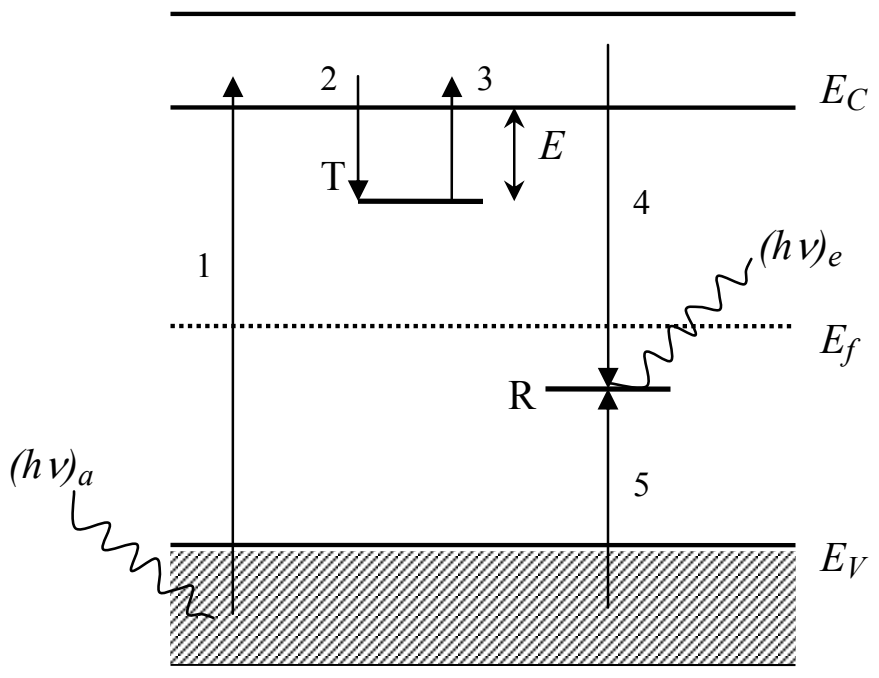

Figure 4.3. Simple two-level model for thermoluminescence. Allowed transitions: (1) ionization after absorption of light; (2) and (5) trapping; (3) thermal release; (4) radiative recombination and the emission of light. Electrons are the active carriers, but an exactly analogous situation arises for holes.

Direct recombination of electrons and holes across the band gap is a less likely process than indirect recombination at a localized defect state, especially in insulators and wide band gap semiconductors. Thus, in order for recombination to occur, holes first 
become trapped at centers (R) (transition 5). Recombination takes place via the annihilation of the trapped holes by free electrons (transition 4). The free electrons may also become trapped at level $\mathrm{T}$ (transition 2 ) in which case recombination can only take place if the trapped electrons absorb enough energy $E$ to be released back into the conduction band, from where recombination is possible. Thus, the luminescence is delayed by the amount of time spent by the electrons in the gap, given by the Arrhenius equation (4.1) and written here as

$$
p=\tau^{-1}=s \exp (-E / k T)
$$

Here $p$ is defined as the probability per unit time of the release of an electron from the trap.

If the trap depth $E$ is such that at the temperature of irradiation $T_{0}, E>k T_{0}$, then any electron which becomes trapped will remain so for a long period of time. Then, even after removal of the radiation there will exist a substantial population of trapped electrons. Furthermore, because the free electrons and holes are created in pairs and are annihilated in pairs, there must be an equal population of trapped holes at level R. The recombination of electrons and holes can be speeded up by raising the temperature above $T_{0}$ such that $E \leq k T$. This in turn will increase the probability of detrapping $p$ and the electrons will be released at a faster rate from the trap into the conduction band. Thermoluminescence now results when the free electrons recombine with the trapped holes (Transition 4).

The intensity of the thermoluminescence $I(t)$ at any time during heating is proportional to the rate of recombination of holes and electrons at level R. If $n_{h}$ is the concentration of trapped holes then

$$
I(t)=-\mathrm{d} n_{h} / \mathrm{d} t
$$

As the temperature is raised the electrons are released and recombination takes place, thus reducing the concentration of trapped holes and increasing the thermoluminescence intensity. As the electron traps are progressively emptied the rate of recombination 
eventually decreases and the thermoluminescence intensity decreases accordingly. This produces the characteristic thermoluminescence peak. Usually, in a thermoluminescence experiment, the temperature is raised as a linear function of time according to

$$
T=T_{0}+\beta t
$$

where $\beta$ is the heating rate given by $\mathrm{d} T / \mathrm{d} t$.

In these thermoluminescence experiments, the most informative results are obtained by simultaneously measuring the intensity of the thermoluminescence as a function of both sample temperature and emission wavelength. For the present study, the experimental setup used for the thermoluminescence experiments is represented schematically in Figure 4.4. The TL data were taken using a spectrograph (Oriel MS260i)

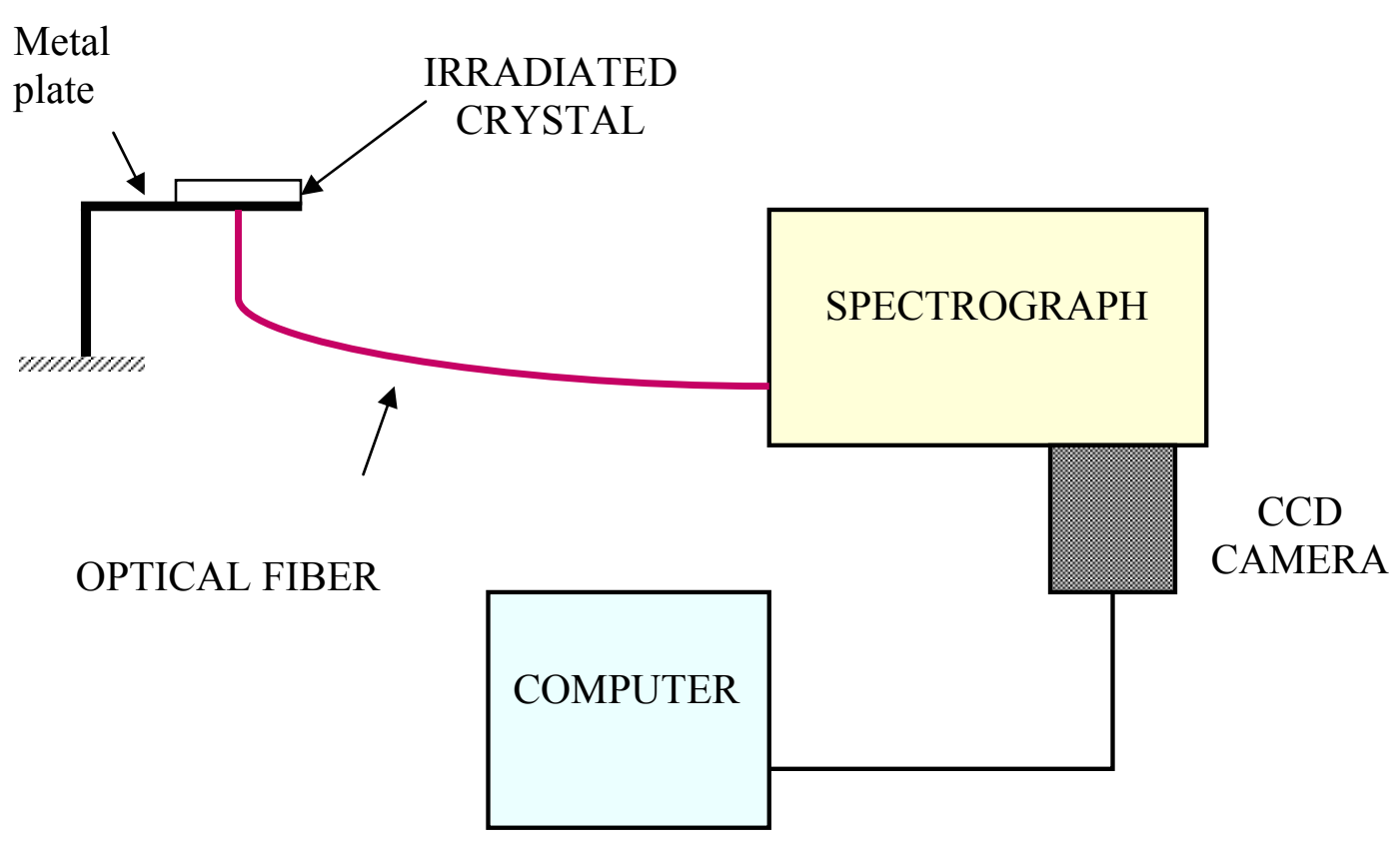

Figure 4.4. Setup for the thermoluminescence experiment. 
coupled to a CCD (charged-couple-device) camera (Oriel InstaSpec IV). The data were then collected and processed by a computer. The spectrograph was wavelength calibrated using a small $\mathrm{Hg}$ (Ar) lamp, and a 20-W quartz-tungsten-halogen lamp was used to correct for the system response.

The crystals used in the TL study, $\mathrm{LiNbO}_{3}$ and $\mathrm{LiTaO}_{3}$, have approximately the same size, $1 \times 3 \times 10 \mathrm{~mm}^{3}$. Prior to the heating and actual data acquisition, the samples were held in liquid nitrogen and irradiated for about 3-5 minutes. Several types of irradiation were used: $60-\mathrm{kV} \times$ rays or ultraviolet laser beams of $266-\mathrm{nm}$ or $355-\mathrm{nm}$ harmonics from a pulsed Nd:YAG or 325-nm from a cw He-Cd. All the excitations used produced essentially the same TL results. After turning off the excitation source, the sample was immediately removed from the liquid nitrogen using hand tweezers and quickly placed on a small horizontal metal plate that was in air and initially at room temperature. The temperature of the sample increased rapidly (from $80 \mathrm{~K}$ to $273 \mathrm{~K}$ in approximately 18 to $20 \mathrm{~s}$ ) as thermal energy flowed into the crystal from the surrounding air and metal plate. Since a thermocouple was not used, we determined when the sample reached $273 \mathrm{~K}$ by noting the time that elapsed before the frost on the crystal (formed by moisture in the surrounding air) turned to water. The light emitted during warming was collected by an optical fiber whose end was positioned less than one millimeter from the sample (no collecting and coupling lenses were used). This method of producing and monitoring TL is simple in design and gave very reproducible results. The rate of warming was approximately linear, and we estimate that the temperature scale in our TL data is accurate to within $\pm 5 \mathrm{~K}$. This uncertainty in temperature is quite adequate for this study since we do not attempt to extract activation energy and kinetic order.

In the elementary, uncomplicated model of two-levels described in Figure 4.3, only one thermoluminescence glow peak should be observed upon heating a specimen following its irradiation. Furthermore, the TL emission should be of one basic color. Even though this is not the case for the majority of phosphorus materials, in this study the 
simple two-level model can be used to explain the single glow peak for $\mathrm{LiNbO}_{3}$ with only one broad emission. However, in the case of $\mathrm{LiTaO}_{3}$, the TL results imply that a slightly different scenario must be considered.

\subsection{Electron Paramagnetic Resonance}

Electron paramagnetic resonance (EPR) is a powerful, versatile, and nondestructive spectroscopic technique. This method has a wide range of application areas (e.g., physics, chemistry, biology, medicine, etc) and is an ideal complementary technique for other methods. A list of commonly used EPR applications include: (a) paramagnetic point defects in single crystals (such as $F$ centers, electrons, or holes at suitable trapping sites, vacancies, antisites, transition metal ions, rare-earth ions); (b) free radicals in solid, liquid, or gaseous phases; (c) acceptors and donors in semiconductors; (d) systems with more than one unpaired electron (like triplet-state systems, biradicals, multiradicals).

In an EPR experiment, an applied magnetic field interacts with the magnetic moment of electrons and nuclei. Like other spectroscopy methods, EPR gives a measure of the energy difference between atomic states. These energy differences, $\Delta \mathrm{E}$, can be measured based upon an important relationship between $\Delta \mathrm{E}$ and the absorption of electromagnetic radiation. According to Planck's law, electromagnetic radiation will be absorbed if

$$
\Delta \mathrm{E}=\mathrm{h} v
$$

where $\mathrm{h}$ is Planck's constant and $v$ is the frequency of the radiation. The absorption of energy causes a transition from the lower energy state to the higher energy state. In conventional spectroscopy, the frequency is varied or swept and the frequencies at which absorption occurs correspond to the energy differences of the states. In the EPR spectroscopy the electromagnetic radiation frequency is held constant and the magnetic field is varied or swept. For EPR experiments, the frequency of the radiation is in the gigahertz range. 
The energy differences studied in EPR spectroscopy are predominately due to the interaction of unpaired electrons with a magnetic field. The applied field contains one component that is time independent (of amplitude $\mathrm{H}_{0}$ ) and one that is time dependent (of amplitude $\mathrm{H}_{1}$ ).

$$
\overrightarrow{\mathrm{H}}=\overrightarrow{\mathrm{H}}_{0} \hat{z}+\overrightarrow{\mathrm{H}}_{1}(\hat{x} \cos \omega t+\hat{y} \sin \omega t) \quad, \quad \mathrm{H}_{1}<<\mathrm{H}_{0}
$$

Consider the simplest energy-level scheme for a particle of spin $1 / 2$ in the magnetic field. In the absence of a static field, the particle can occupy either of two energetically equivalent states (spin degenerate states). The constant (time independent) component of the applied magnetic field interacts with the magnetic dipole moment of the unpaired electron, thus lifting the spin degeneracy and producing two energy levels with spin states, conventionally called, "spin up" ( $\alpha$ state), denoted by $M_{S}=+1 / 2$ and "spin down" ( $\beta$ state), denoted by $M_{S}=$ $-1 / 2$. These energy levels are referred to as the Zeeman energy levels and the difference between these energy levels is called the Zeeman splitting. In general, for a system with total spin $\mathrm{S}, \mathrm{M}_{\mathrm{S}}$ can take the quantized values $-\mathrm{S},-\mathrm{S}+1, \ldots, \mathrm{S}-1, \mathrm{~S}$ and the total number of energy levels is given by $(2 S+1)$. The energy of the magnetic dipole moment $(\mu)$ in a magnetic field $(H)$ can be written as

$$
E=-\vec{\mu} \cdot \vec{H}
$$

This scalar product of the two vectors would be proportional to the cosine of the angle between the magnetic moment and the applied field, but the electron magnetic moment is quantized and this product can take only certain values. Considering $\mathrm{H}$ to be in the $z$ direction, the component of electron spin magnetic moment along the $z$ axis is determined by

$$
\mu_{z}=-g \beta M_{S}
$$


where $\mathrm{g}$ is the $\mathrm{g}$-factor, a proportionality constant that varies from one paramagnetic system to another. It is approximately equal to 2.00232 for free electrons, but can shift from this value depending on the electronic configuration of the ion and on the local crystal environment. The constant $\beta$ is the Bohr magneton, which is the natural unit of electronic magnetic moment. $\mathrm{M}_{\mathrm{S}}$ has quantized values determined by the spin (S) of the system.

Combining equations (4.7) and (4.8) with the condition that the static magnetic field $\mathrm{H}_{0}$ is along the $\mathrm{z}$ axis results in a set of basic equations for EPR:

and

$$
E=g \beta H_{0} M_{S}= \pm \frac{1}{2} g \beta H_{0}
$$

$$
\Delta E=E_{\alpha}-E_{\beta}=h v=g \beta H_{r}
$$

Equation (4.9) gives the Zeeman energy levels and equation (4.10) represents the resonance condition. In equation (4.10), $v$ is the frequency of the microwave radiation and $\mathrm{H}_{\mathrm{r}}$ is the static magnetic field at which the resonance condition is met. Equation (4.10) implies that this EPR transition is "allowed" if the selection rule $\Delta \mathrm{M}_{\mathrm{S}}= \pm 1$ is satisfied. A schematic diagram of this simple situation is provided in Figure 4.5. Energy is represented by the vertical axis. The upward and downward transitions indicated in Figure 4.5 correspond to $\Delta \mathrm{M}_{\mathrm{S}}=+1$ and $\Delta \mathrm{M}_{\mathrm{S}}=-1$, respectively.

From the simple resonance condition one can easily determine the $\mathrm{g}$ factor by

$$
g=\frac{h v}{\beta H_{r}}
$$

This is applicable for isotropic spin systems where the $\mathrm{g}$ factor is independent of field direction.

Two facts are apparent from equations (4.9) and (4.10) and from Figure 4.5: (1) the two spin states have the same energy in the absence of a magnetic field (they are degenerate) and without a static magnetic field there is no energy difference to measure, and (2) the measured energy difference depends linearly on the magnetic field for an $S=1 / 2$ system with 
no hyperfine.

In addition to the contribution of the time independent magnetic field, the timevarying component (equation (4.6)), with much smaller amplitude than the constant magnetic field, is produced by the microwave radiation and drives the transition (absorption) between the two energy levels.

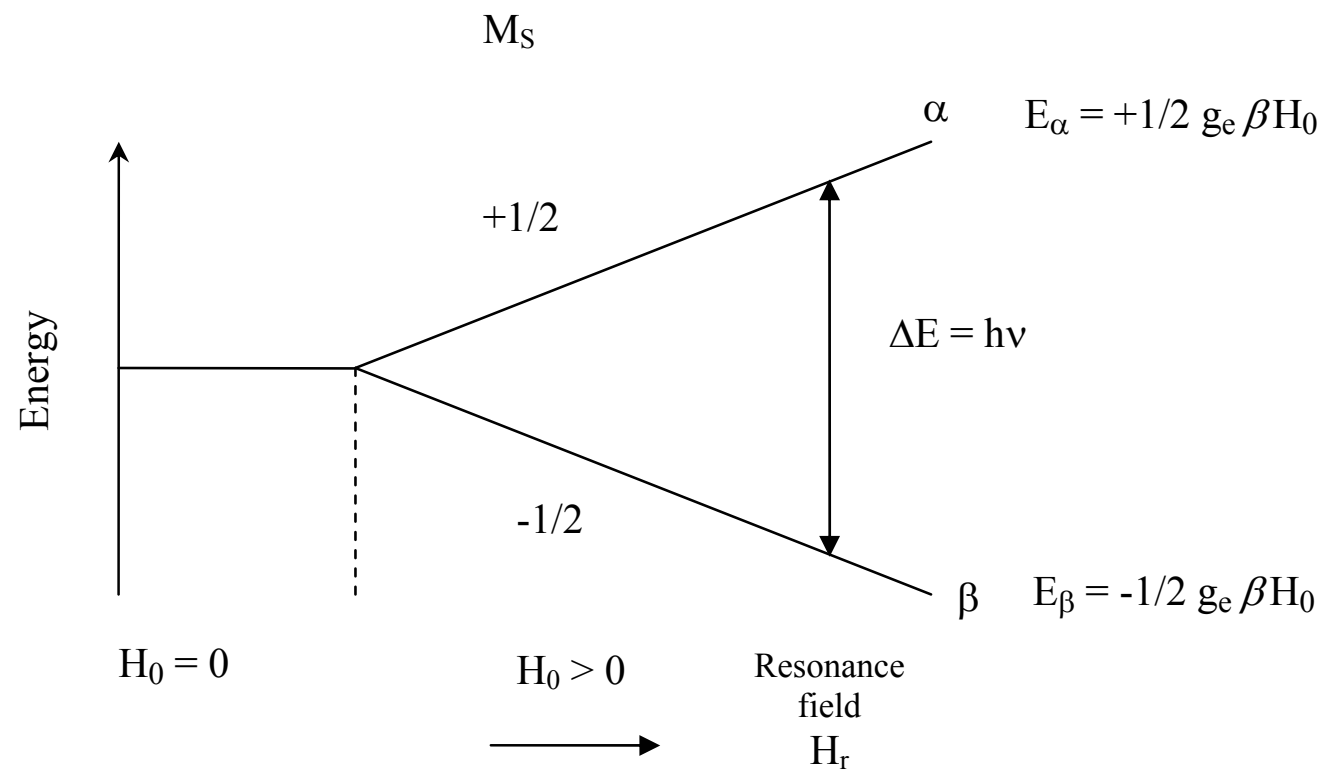

Figure 4.5. Energy-level diagram for the simplest system, as a function of applied magnetic field $H$, showing EPR absorption. $E_{\alpha}$ and $E_{\beta}$ represent the energies of $M_{S}=+1 / 2$ and the $M_{S}=-1 / 2$.

As long as $\mathrm{H}_{0}>>\mathrm{H}_{1}$, the time-varying magnetic field can be considered as a contribution to the perturbation Hamiltonian $\hat{\mathcal{H}}^{\prime}$

$$
\hat{\mathcal{H}}^{\prime}=-\vec{M} \cdot \vec{H}_{1}=\frac{g \beta}{\hbar} \vec{H}_{1}\left(\hat{S}_{x} \cos \omega t+\hat{S}_{y} \sin \omega t\right)
$$

and we can apply Fermi's Golden Rule to determine the transition probability $\left(\mathrm{R}_{\mathrm{f} \rightarrow \mathrm{i}}\right)$ between the spin states: 


$$
R_{f \rightarrow i} \propto\left|\left\langle\mp \frac{1}{2}\left|\hat{\mathcal{H}}^{\prime}\right| \pm \frac{1}{2}\right\rangle\right|^{2} \propto\left|\left\langle\mp \frac{1}{2}\left|\hat{S}_{x} \cos \omega t+\hat{S}_{y} \sin \omega t\right| \pm \frac{1}{2}\right\rangle\right|^{2}=1
$$

Therefore, by applying the microwave field perpendicular to the static field, the transition probability is maximized. From eq. (4.13) we see that the selection rule for "allowed" magnetic dipole transition is $\Delta \mathrm{M}_{\mathrm{S}}= \pm 1$. Transitions for which this selection rule are not satisfied are called "forbidden." This is an ideal situation since, in practice, the actual spin states may be linear combinations of the basis states. In such cases, the definitions of "allowed" and "forbidden" transitions are no longer well define. Applying the microwave field $\mathrm{H}_{1}$ parallel to the static field $\mathrm{H}_{0}$ would result in no transition since the matrix $\left\langle\mp \frac{1}{2}\left|S_{z}\right| \pm \frac{1}{2}\right\rangle$ element would not couple the two energy states and the product is zero. A transition takes place only if the coupling through the perturbation Hamiltonian is nonzero.

Experimentally, the EPR spectrum does not represent the absorption band but the first derivative of the absorption. This is measured using phase-sensitive lock-in detection and magnetic field modulation. A typical EPR absorption line is shown in Figure 4.6 (a) and the corresponding first derivative is given in Figure 4.6 (b). This is a suggestive representation,

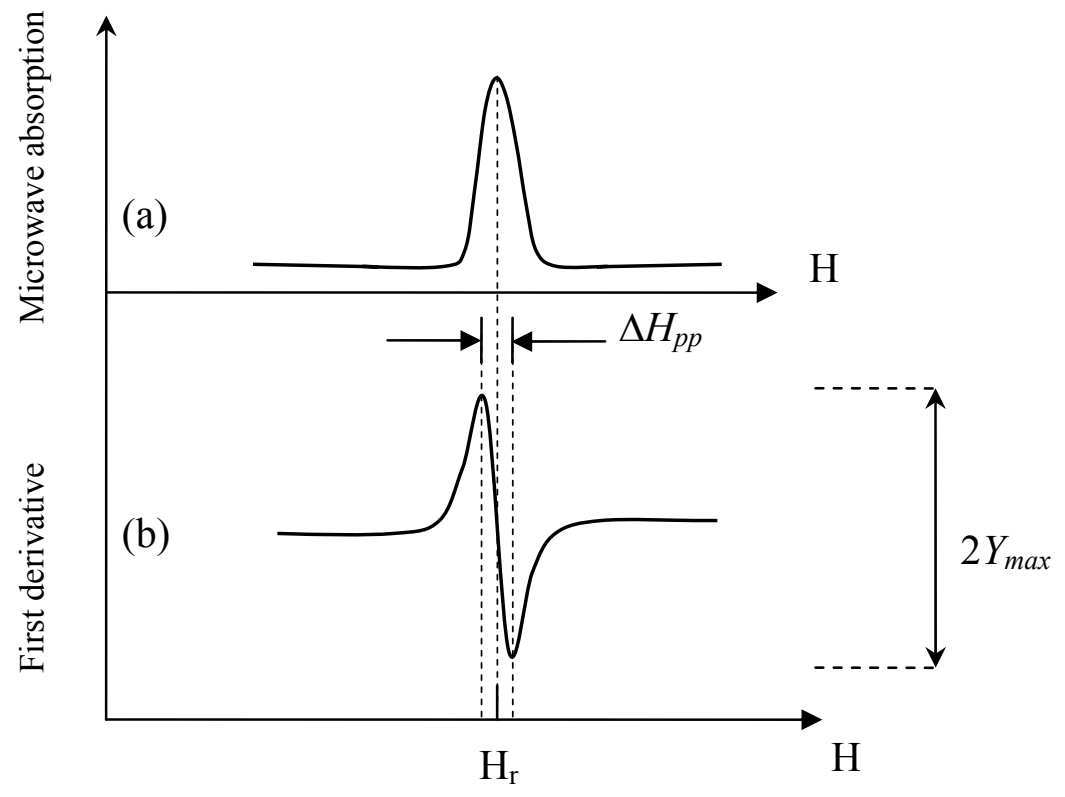

Figure 4.6. (a) Microwave absorption band at $\mathrm{H}=\mathrm{H}_{\mathrm{r}}$. (b) The EPR signal is the first derivative of the absorption spectrum with the center at resonant field $\mathrm{H}_{\mathrm{r}}$. 
not an exact one. Theoretically, the line shape of an EPR absorption band should be Lorentzian, but in practice, the line shape of the absorption band is very often Gaussian. This is because of unresolved hyperfine interaction. In some cases, there is a combination of lorentzian and gaussian line shapes. The relative contribution from the two line shapes is important information when determining the relative concentration of centers from their EPR lines. It is also often of considerable interest to determine the relative intensity of different lines in a spectrum. The most accurate procedure is to integrate the full absorption curve. However, if the line widths of two components are equal, then peak-to-peak amplitudes of the derivative lines will be proportional to their intensities. Even when line widths are different, the approximate relative intensity $I$ of a line may be obtained from the expression:

$$
I \propto Y_{\max }\left(\Delta H_{p p}\right)^{2}
$$

where $2 Y_{\max }$ is the peak-to-peak derivative amplitude and $\Delta H_{p p}$ is the peak-to-peak width [46]. When a knowledge of the absolute concentration of paramagnetic centers is desired, determining the absolute intensity of a signal depends on many factors and thus many errors might be introduced. Among these factors are: the modulation amplitude, the spectrometer gain, the sample temperature, the amplitude of the microwave magnetic field $\mathrm{H}_{1}$ at the sample, the spin of the paramagnetic species, the microwave frequency, and the $\mathrm{g}$ factor.

Also, in magnetic resonance, the absorption line intensity, or the peak-to-peak derivative amplitude, is directly proportional to the magnetic susceptibility $\chi$ and from the Curie law variation of $\chi$ :

$$
\chi=\frac{N \mu_{p}^{2}}{3 k_{B} T}=\frac{C}{T}
$$

(where $C$ is the Curie constant, and $T$ is the temperature), we see that the resonance varies as $1 / \mathrm{T}$ and it is directly proportional to $\mathrm{N}$, the number of ions which are responsible for the signal. Thus, as the temperature decreases, the EPR signal increases. This is often desired and it is achieved by cooling the sample to liquid-helium temperatures. 
It is also important to note that the probability of a transition from $\mid-1 / 2>$ to $<+1 / 2 \mid$ is equal to the probability of a transition from $\mid+1 / 2>$ to $<-1 / 2 \mid$ (i.e., transition rates for stimulated absorption and emission are equal). For this reason it is necessary to have a population difference between the two spin states in order to observe an EPR signal. This population distribution is called spin polarization and is proportional to the difference in population between the spin states

$$
P=\frac{n_{\downarrow}-n_{\uparrow}}{n_{\downarrow}+n_{\uparrow}}
$$

Typically, a population difference of only a few percent is enough to measure a net absorption. At low temperatures, where one can take advantage of the thermalized Boltzmann distribution of electrons, a significant population difference can be achieved. Then again, when the temperature becomes too low, the time it takes an excited electron to release its energy to the lattice and to return to its ground state (called the spin-lattice relaxation time) becomes very long. Consequently, the onset of resonance will quickly destroy the spin population difference, resulting in minimal net absorption. This effect is called temperature saturation. Similarly, if a too large microwave power is used so that spins are excited more rapidly than they relax, an effect called power saturation will occur. For these reasons, it is necessary to carefully determine the optimal temperature and power for resonance in many systems.

The EPR results presented here were taken with a Bruker Instruments ESP-300 spectrometer operating near $9.45 \mathrm{GHz}$. An Oxford Instruments Model ESR-900 helium-gas flow system maintained the sample at selected temperature in the 20-300 K range during the EPR experiments. 


\subsection{Optical and infrared absorption}

In optical absorption spectroscopy, electromagnetic radiation in the near ultraviolet, visible, or near infrared regions is used to excite transitions between the electronic states. Experimentally, an absorption spectrum is determined by varying the wavelength of the incident radiation and recording the intensity of the transmitted beam. Experimental measurements are presented as graphs of absorbed intensity versus photon energy $(E=h v)$, frequency $(v)$ or wavelength $(\lambda)$. A typical setup for recording absorption spectra contains a broad band light source, a monochromator, and a detector.

In the present study, a Cary Model 14 spectrophotometer was used for ultraviolet and visible measurements. This unit had been modernized by Olis, Inc. A schematic optical plan is given in Figure 4.7. The drawing is not to scale and does not reproduce exactly the model used, but it shows a basic setup for measurements of optical absorption. The Cary 14 spectrometer uses a tungsten lamp for the visible excitation region and a deuterium lamp for the ultraviolet region. This model employs a double monochromator. The energy receiver is a photomultiplier tube (PMT) for both the ultraviolet and visible radiation. After being dispersed by a prism and a grating, the light is alternately sent through the reference cell and the sample cell by means of a rotating semicircular mirror and a chopper. In this dual-beam setup, the light is passed alternately through the reference and the sample by using a chopper. The PMT receives light from only one beam at a time. The monochromator range is from $186 \mathrm{~nm}$ to $2600 \mathrm{~nm}$, but I have used only the range: $200 \mathrm{~nm}$ to $780 \mathrm{~nm}$ for all the spectra collected. The experiment is done as follows: an absorption spectrum is recorded with no sample in the spectrometer, and this gives the background response of the instrument. Then a sample is introduced inside the sample chamber (cell) and another spectrum is taken. The difference between these two spectra is the real absorption spectrum given by the sample under study. 


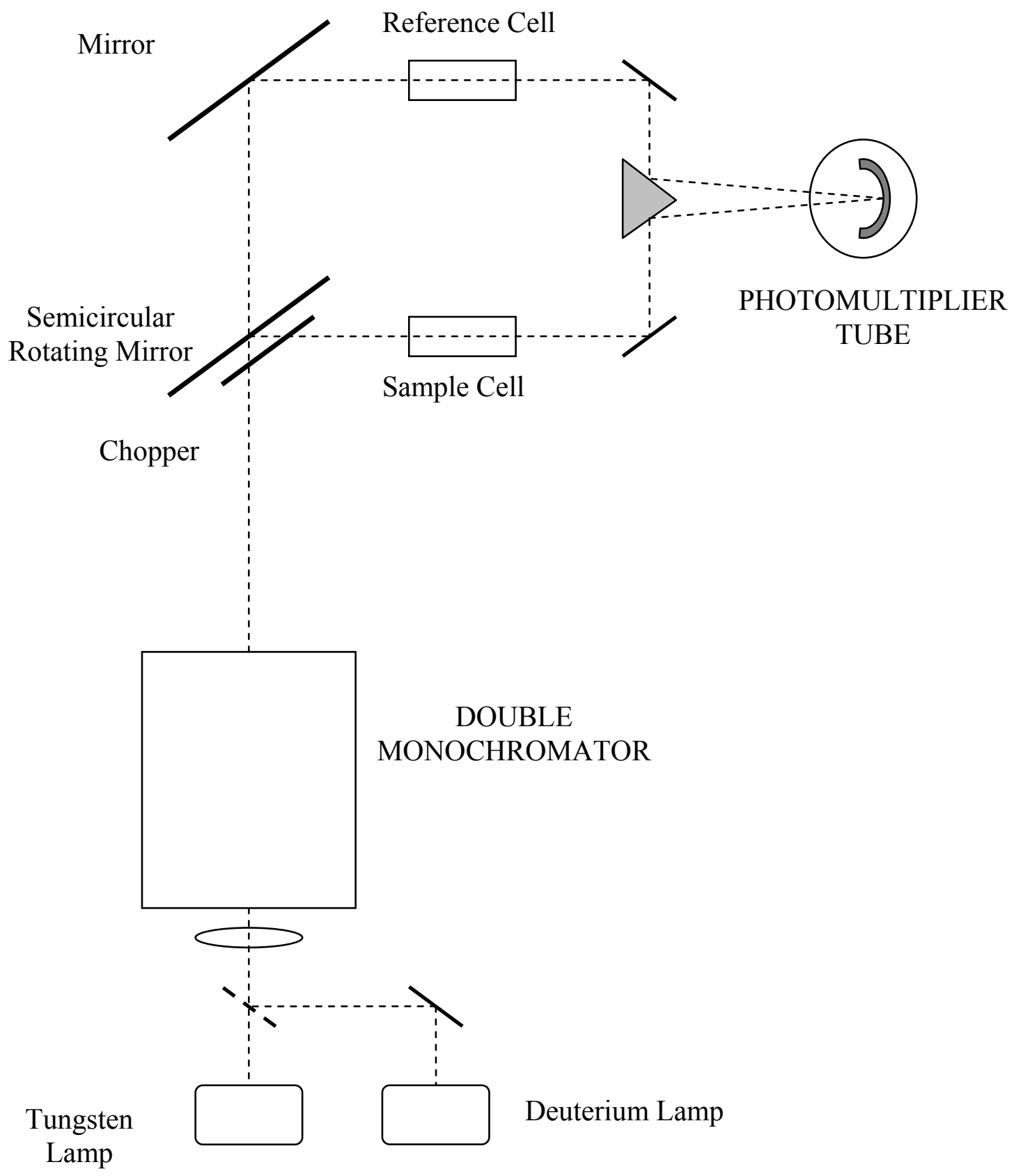

Figure 4.7. Setup for optical ultraviolet and visible absorption measurements. 
The measured absorbance is expressed in units of optical density (OD), which is defined by

$$
O D=\log _{10}\left(\frac{1}{T}\right)=\log _{10}\left(\frac{I_{0}}{I}\right)
$$

where

$O D$ is the optical density,

$\mathrm{T}$ is the sample transmission,

$\mathrm{I}_{0}$ is the incident light intensity, and

I is the transmitted light intensity.

All the spectra recorded are presented using OD on the vertical axis and wavelength or wavenumber on the horizontal axis. In general, the absorption coefficient can be obtained from the Lambert - Beer law which says that the attenuation of a light beam traveling through a light-absorbing medium is described by the equation

$$
I=I_{0} e^{-\alpha x}
$$

where $\mathrm{I}$ is the intensity of the transmitted beam,

$\mathrm{I}_{0}$ is the intensity of the incident beam,

$\alpha$ is the absorption coefficient of the medium, and

$\mathrm{x}$ is the length of the light path in the sample or the sample thickness.

From equations (4.17) and (4.18), we see that the absorption coefficient is

$$
\alpha=\frac{O D}{x \log e}
$$

Equation (4.19) is valid when reflective losses are small and we can neglect them. A more precise mathematical formula for the absorption coefficient which contains corrections for reflective losses is given by

$$
\alpha=\frac{1}{x \log e}[O D+2 \log (1-R)]
$$


where $\mathrm{R}$ represents the reflective losses that occurs at each interface air/sample for zero incidence angle and the other variables are defined above. If the index of refraction of the crystal is known, the reflective losses can be calculated from

$$
R=\left(\frac{n-1}{n+1}\right)^{2}
$$

When available, the index of refraction can be calculated from the Sellmeier equations.

The optical absorption data in the near infrared region were recorded using a Thermo Nicolet (Nexus 870) Fourier-transform infrared (FTIR) spectrometer. The infrared light coming from a source (a lamp) is directed into the Michelson interferometer, the key component of FTIR spectrometry. In the interferometer, the light beam strikes the beamsplitter and about half of the light is reflected and directed onto the fixed mirror. The remainder of the light is transmitted through the beamsplitter and is directed onto the moving mirror. When the beams recombine, constructive and destructive interference occurs depending on the position of the moving mirror relative to the fixed mirror. When both mirrors are the same distance from the beamsplitter, the two reflected beams pass through exactly the same pathlength and consequently, are totally in phase. The resulting signal intensity is at its maximum. The modulated beam is reflected from mirrors to the sample, where selective absorption takes place. From the sample the beam travels on to the detector, which translates the beam into an electrical signal. The signal from the detector is sent to a computer and converted into an IR spectrum using software programs designed to quickly evaluate the Fourier Transform (FT) of the interference spectrum received from the detector.

The infrared absorption spectra were recorded in the range $(900-3500 \mathrm{~nm})$, which is considered part of the near-infrared range. For the best detection of a signal in this region I have used a combination of a white light source, $\mathrm{a} \mathrm{CaF}_{2}$ beamsplitter, and a MCT detector. Since the absorption bands I observed in this region are broad, a resolution of $16 \mathrm{~cm}^{-1}$ was appropriate for taking these data. The near-infrared absorption bands measured for lithium 
niobate and lithium tantalate crystals represent induced absorption bands. The bands were formed when ultraviolet light was incident on the crystals while they were held at cryogenic temperature $(10 \mathrm{~K})$. To measure low temperature spectra, the crystals studied were mounted in an Optistat system manufactured by Oxford Instruments, Inc. In this system, flowing helium vapor allows variation of sample temperature over the 10 to $296 \mathrm{~K}$ range. 


\section{CHAPTER 5}

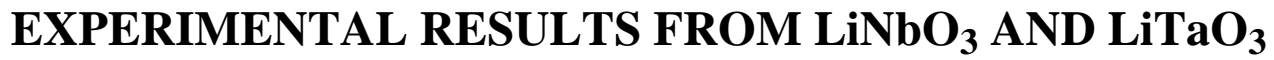

\section{1. $\mathrm{LiNbO}_{3}$}

\subsubsection{TL, EPR, and optical absorption results}

In this section, I describe the thermoluminescence, optical absorption, and electron paramagnetic resonance results obtained from $1 \% \mathrm{Mg}$-doped stoichiometric $\mathrm{LiNbO}_{3}$ crystals. These crystals differ from the more widely studied congruent $\mathrm{LiNbO}_{3}$ crystals in one major respect. In congruently grown crystals, niobium ions occupy lithium sites and thus provide charge compensation for the large numbers of lithium vacancies that are present. In the $1 \%$ Mg stoichiometric crystals, there are very few niobium ions on lithium sites and, instead, the divalent magnesium ions on lithium sites provide charge compensation for the smaller number of lithium vacancies that are present. Overall, the concentrations of point defects are considerably less in $1 \% \mathrm{Mg}$ stoichiometric crystals compared to congruent crystals. This is reflected in the stoichiometric crystals by narrower EPR lines in the spectra of transition-

metal-ion impurities such as $\mathrm{Fe}^{3+}$ (i.e., linewidths are reduced as the random strain in the crystal is removed [49]).

The ability to detect emitted light from the $1 \% \mathrm{Mg}$-doped stoichiometric crystals directly correlates with the lack of niobium antisite defects. A bright blue (440 nm) thermoluminescence peak at $94 \mathrm{~K}$ was easily observed in the case of the $\mathrm{Mg}$-doped stoichiometric crystals, while similar experiments on congruent $\mathrm{LiNbO}_{3}$ crystals gave no observable TL results. First the optical absorption data will be presented. Figure 5.1 shows 
representative optical absorption spectra from one of our $1 \% \mathrm{Mg}$ stoichiometric LN crystals.

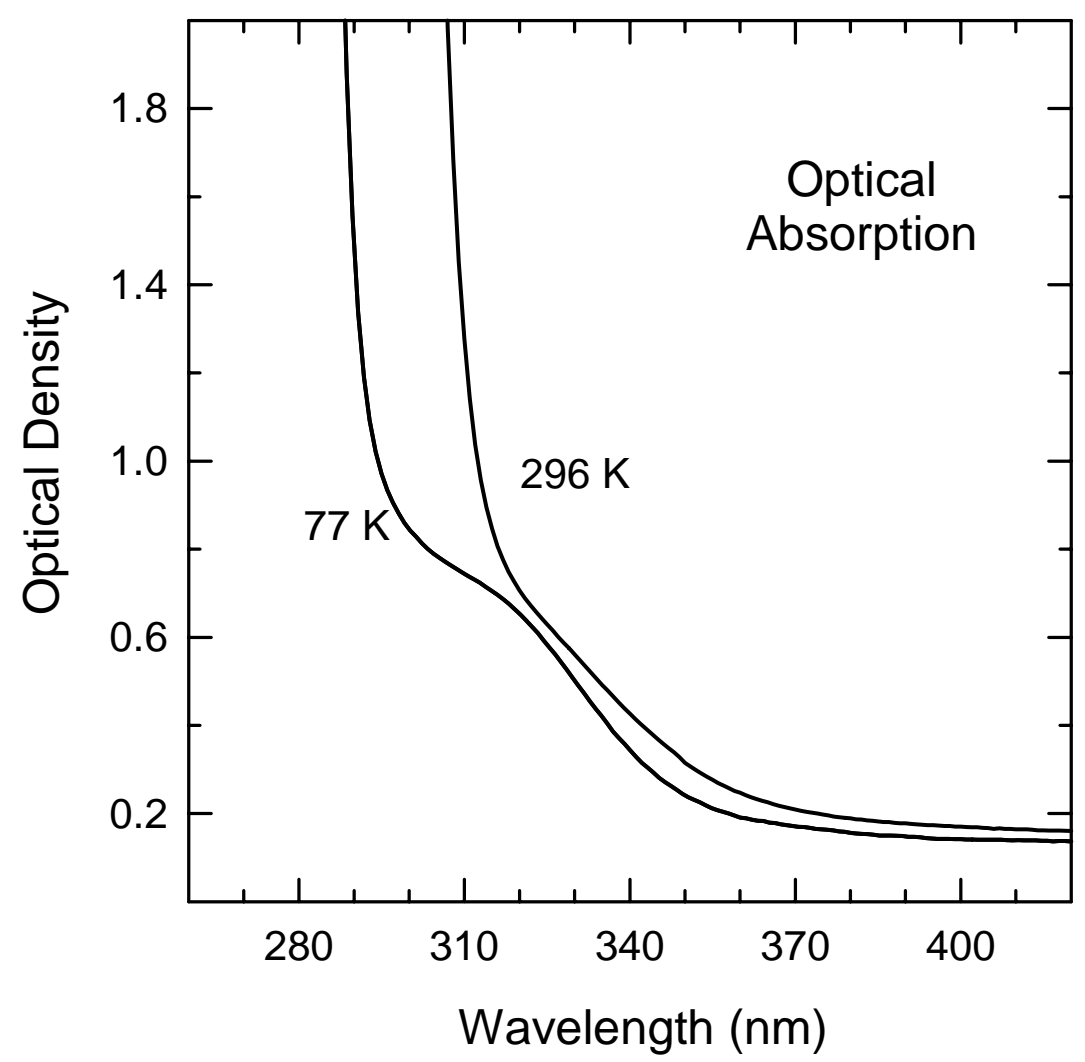

Figure 5.1. Optical absorption from a $1 \% \mathrm{Mg}$ stoichiometric $\mathrm{LiNbO}_{3}$ crystal. These data were taken at room temperature and at $77 \mathrm{~K}$ with unpolarized light propagating along the c axis. An extrinsic absorption band, peaking near $320 \mathrm{~nm}$ and well-resolved at $77 \mathrm{~K}$, is attributed to trace amounts of $\mathrm{Fe}^{3+}$ ions.

These data were taken at room temperature and at $77 \mathrm{~K}$ using unpolarized light propagating along the c axis. The band edge shifts from $308 \mathrm{~nm}$ to $289 \mathrm{~nm}$ as the temperature is lowered. Of even more interest, a near-edge absorption band is barely detected at room temperature, but is easily resolved at $77 \mathrm{~K}$. This latter band has been assigned to a charge-transfer 
transition of $\mathrm{Fe}^{3+}$ ions (i.e., a transition in which an electron moves from an adjacent oxygen ion to the Fe ion) $[47,48]$. Charge-transfer absorption bands have large oscillator strengths and, in the case of stoichiometric $\mathrm{LiNbO}_{3}$, provide a convenient way to monitor the trace amounts of $\mathrm{Fe}^{3+}$ ions usually present. In congruent $\mathrm{LiNbO}_{3}$ crystals, the band edge is at longer wavelengths and the charge-transfer transition of the $\mathrm{Fe}^{3+}$ ions is not as easily detected. Figure 5.2 illustrates the optical absorption spectrum at room temperature of congruent $\mathrm{LiNbO}_{3}$ in comparison with stoichiometric $\mathrm{LiNbO}_{3}$. The band edge at room temperature

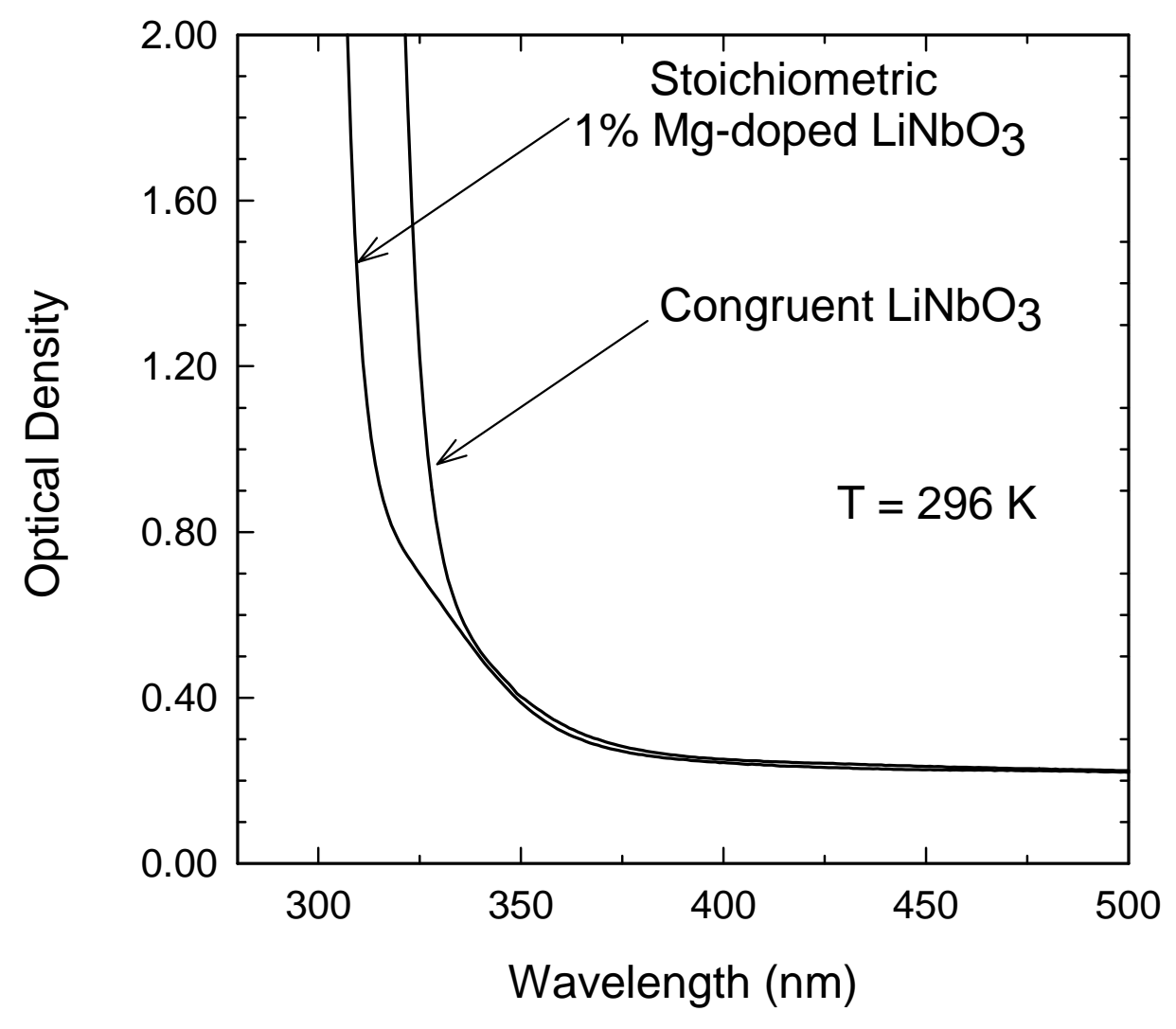

Figure 5.2. Optical absorption from congruent and stoichiometric $\mathrm{LiNbO}_{3}$. These data were taken at room temperature with unpolarized light propagating along the $\mathrm{c}$ axis. The band edge of the congruent crystal is at longer wavelengths and the band due to Fe is not easily detected. 
shifts from 320 to $308 \mathrm{~nm}$ due to changes in the stoichiometry. The measurement of the absorption edge is a sensitive technique to determine the degree of nonstoichiometry in crystals such as $\mathrm{LiNbO}_{3}[10,39,44]$.

In the thermoluminescence (TL) experiments, a sample was initially exposed to $\mathrm{x}$ rays or an ultraviolet laser beam (either $325 \mathrm{~nm}$ or $355 \mathrm{~nm}$ ) while being held at $77 \mathrm{~K}$. Rapid warming then produced a TL "glow" peak near $94 \mathrm{~K}$, with a maximum in its wavelength dependence near $440 \mathrm{~nm}$. Optical absorption in the near-infrared region and electron paramagnetic resonance (EPR) data, taken after similar irradiations, helped us to identify the electron and hole centers participating in the radiative recombination process. During an irradiation, holes are trapped on oxygen ions adjacent to lithium vacancies and a portion of the corresponding electrons are trapped on niobium ions at regular lattice sites (i.e., as shallow trapped electrons). Upon warming, the electrons that are localized on the niobium ions become thermally unstable near $94 \mathrm{~K}$ and move to the trapped holes, where their radiative recombination produces the observed blue emission.

Thermoluminescence results from a Mg-doped stoichiometric $\mathrm{LiNbO}_{3}$ crystal are shown in Figure 5.3. These data are presented in a three-dimensional plot with temperature, wavelength, and intensity as the axes $(I-T-\lambda)$. This coordinated measurement of intensity of the thermoluminescence as a function of both sample temperature and the emission wavelength is a very informative procedure. The results described in Figure 5.3 were obtained after the sample was exposed to the pulsed $355-\mathrm{nm}$ laser beam while being held at $77 \mathrm{~K}$. A rapid rise in temperature then produced the observed TL. The heating rate was approximately $10.7 \mathrm{~K} / \mathrm{s}$ and, a complete measurement of the wavelength dependence of the emission was made every $0.9 \mathrm{~K}$ with the CCD spectrograph and camera.

Cross-sectional views of the data in Figure 5.3 are provided in Figures 5.4 and 5.5. The temperature dependence of the emission, corresponding to a wavelength near the peak, is shown in Figure 5.4. The maximum in the emission occurs near $94 \mathrm{~K}$ and the width of the 


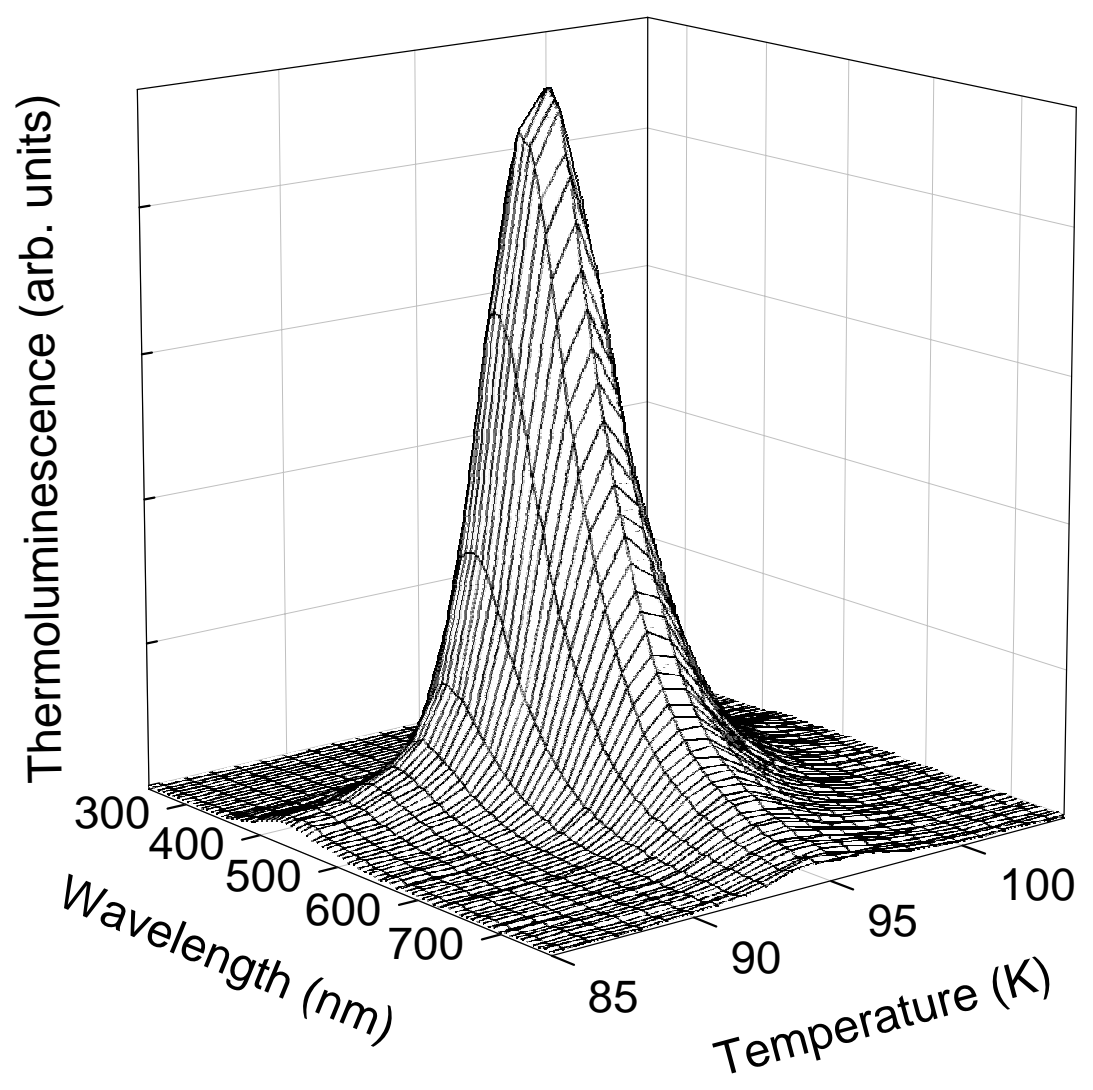

Figure 5.3. Three-dimensional view $(I-T-\lambda)$ of the thermoluminescence data taken from a $1 \% \mathrm{Mg}$ stoichiometric $\mathrm{LiNbO}_{3}$ crystal after excitation at $77 \mathrm{~K}$ with a pulsed 355-nm laser. The intensity of the emission is plotted as a function of both temperature and wavelength.

TL peak is sharp, indicating that the release of charge leading to radiative recombination is a well-defined and discrete phenomenon. As an aside, we note that a slower warming rate, which is more typical in many TL experiments, would cause the peak to shift to a slightly 
lower temperature [2 ]. The wavelength dependence of the emission, monitored at $94 \mathrm{~K}$, is shown in Figure 5.5. These data have been corrected for system response, and they verify that the peak in the emission occurs near $440 \mathrm{~nm}$. In addition to the $355-\mathrm{nm}$ laser beam, two other excitation sources $(60-\mathrm{kV}$ x rays and a $325-\mathrm{nm}$ laser) were used to produce the TL in our $\mathrm{LiNbO}_{3}$ crystals. And, in each case, the TL results were the same as those shown in Figures 5.3, 5.4, and 5.5. During an irradiation, holes are trapped on oxygen ions adjacent to

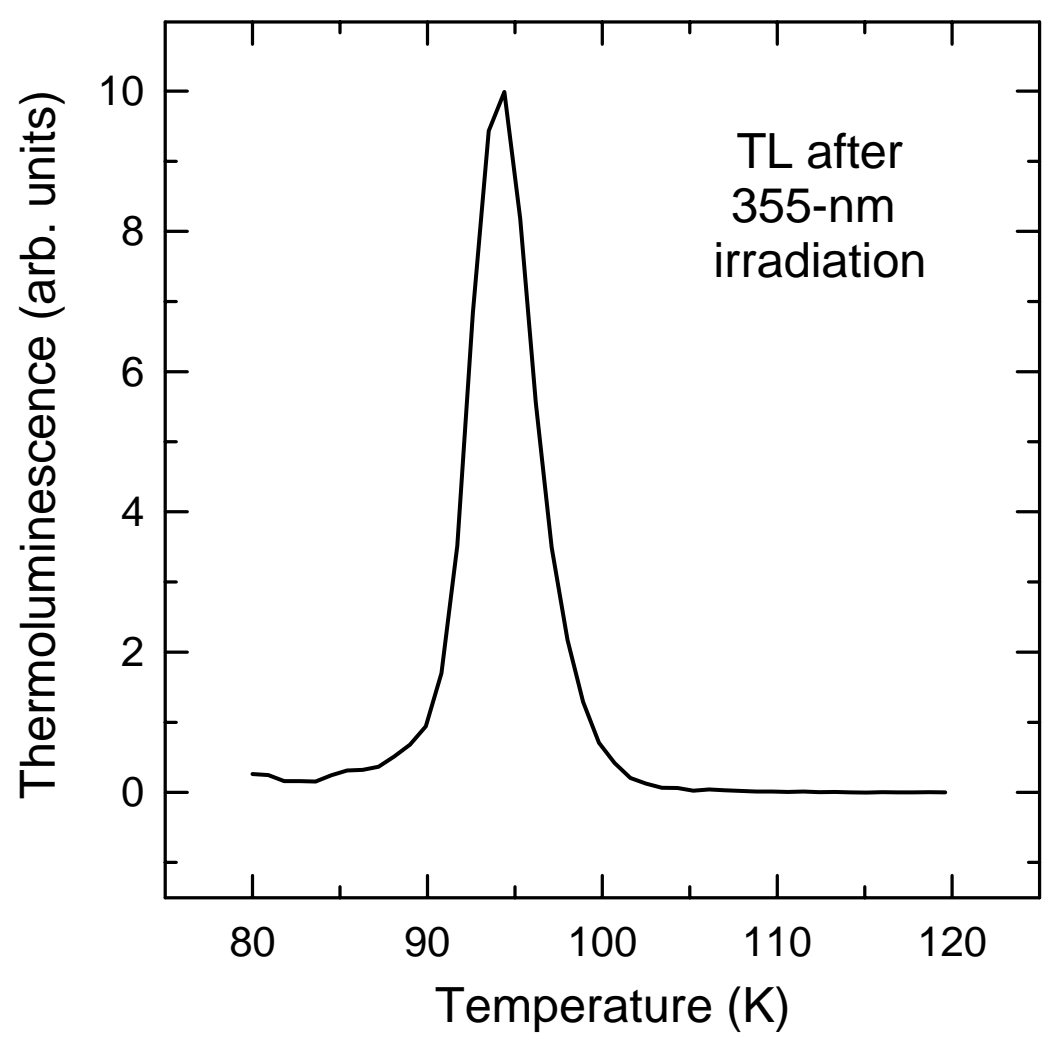

Figure 5.4. A cross section view (taken from the data in Figure 5.3) showing the temperature dependence of the thermoluminescence from $\mathrm{LiNbO}_{3}$. These data correspond to a wavelength of $440 \mathrm{~nm}$ (i.e., the maximum of the emission). The TL peak occurs near $94 \mathrm{~K}$. 
lithium vacancies and a portion of the corresponding electrons are trapped on niobium ions at regular lattice sites (i.e., as shallow trapped electrons). Upon warming, the electrons that are localized on the niobium ions become thermally unstable near $94 \mathrm{~K}$ and move to the trapped holes, where their radiative recombination produces the observed blue emission.

There are two primary goals in an investigation of a TL peak. These are, first, to identify the participating electron and hole traps and, second, to determine the recombination site (in other words, does the electron move to the hole or does the hole move to the electron).

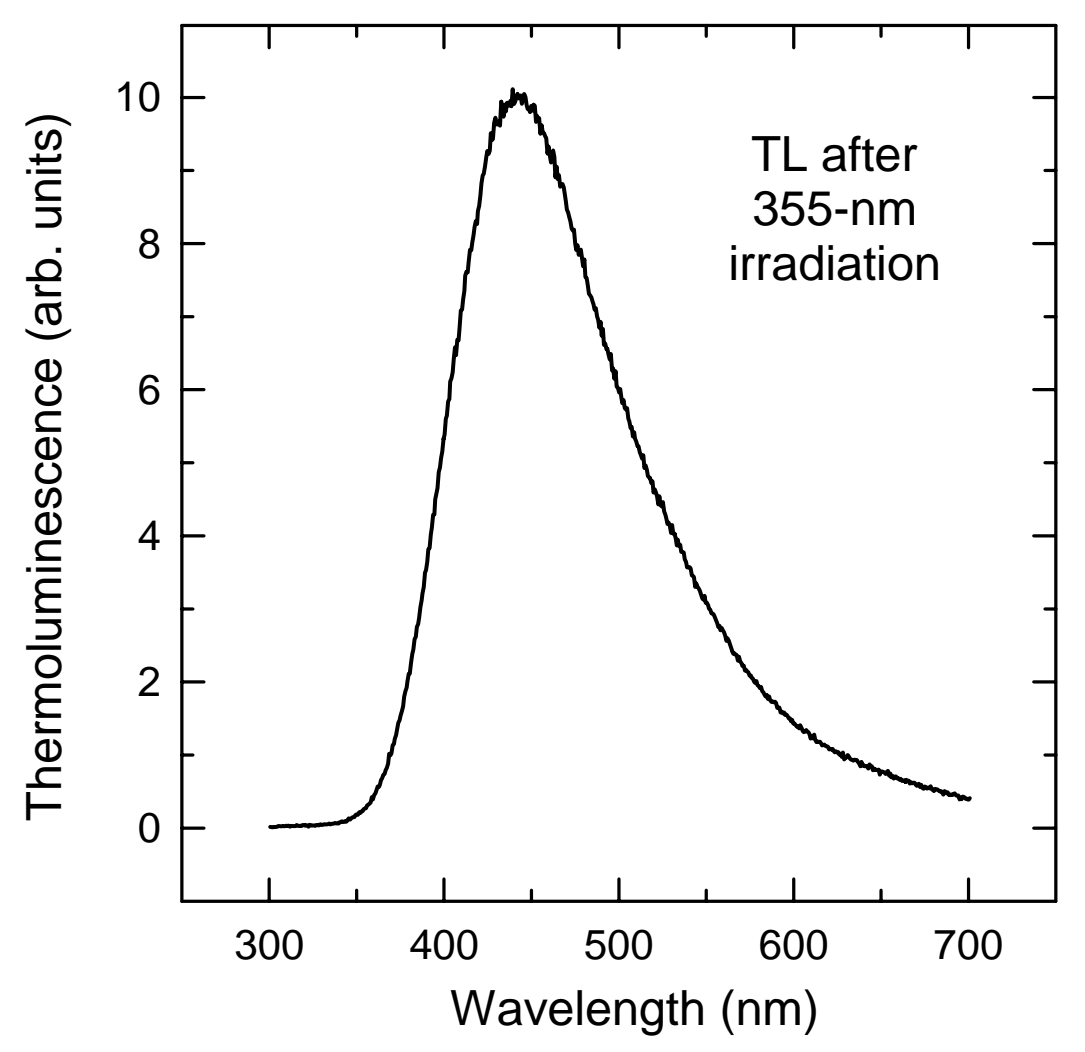

Figure 5.5. A cross-section (taken from the data in Figure 5.3) showing the wavelength dependence of the thermoluminescence from $\mathrm{LiNbO}_{3}$. These data correspond to a temperature of $94 \mathrm{~K}$ (i.e., the maximum of the emission). The TL peak occurs near $440 \mathrm{~nm}$. 
Separate experiments were conducted to help identify the electron and hole centers that are participating in the radiative recombination process described by the data in Figure 5.3. Optical measurements were made at $10 \mathrm{~K}$ in the near-infrared region before and during an exposure of a $1 \% \mathrm{Mg}$ stoichiometric $\mathrm{LiNbO}_{3}$ crystal to the $325-\mathrm{nm}$ laser beam and separately to the 355-nm laser beam. Similar results were observed for both wavelengths. We did not repeat this experiment using $60-\mathrm{kV}$ x rays because of the technical problems associated with introducing these high-energy $x$ rays into the optical cryostat. The absorption band induced

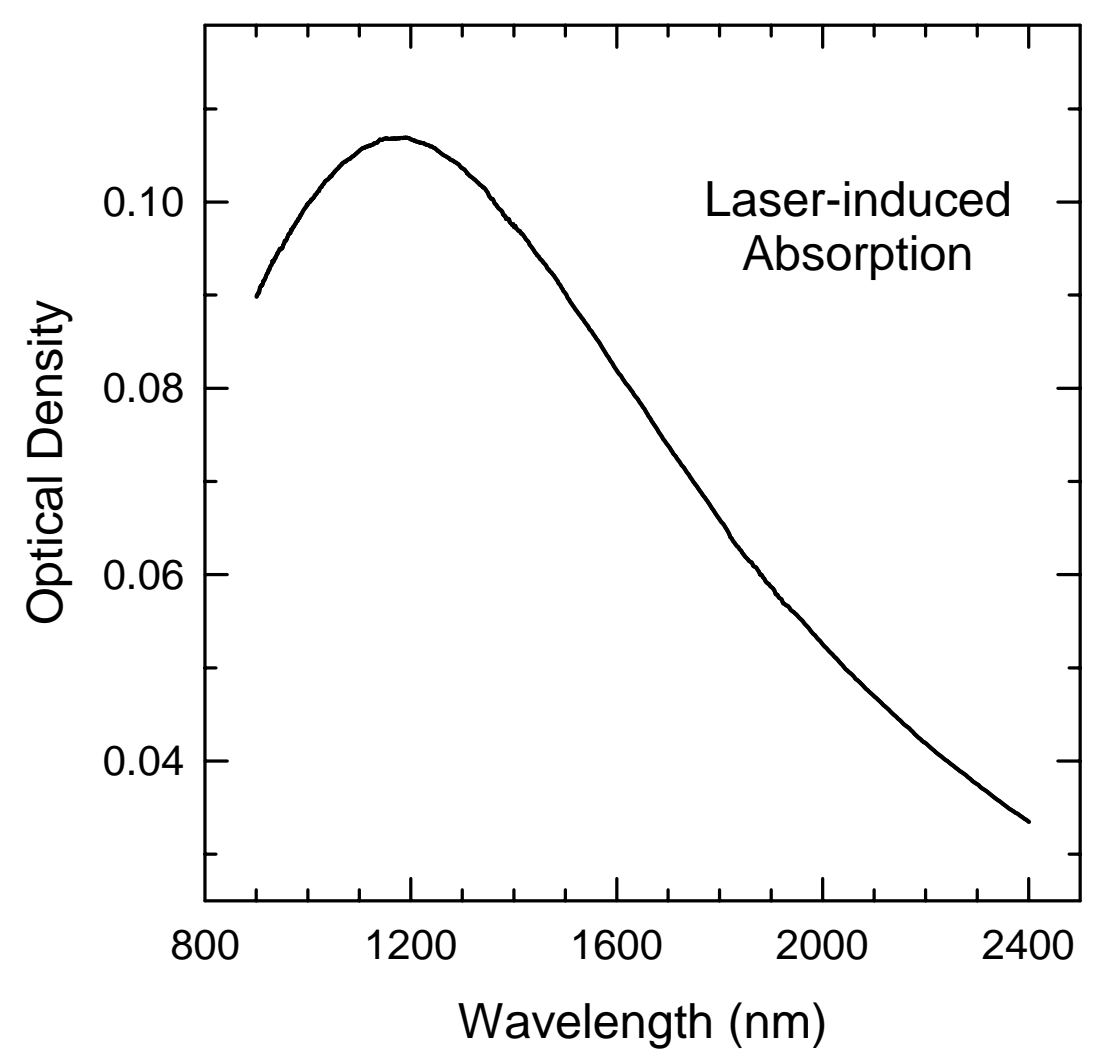

Figure 5.6. Optical absorption from the shallow electron trap, produced at $10 \mathrm{~K}$ by a 325-nm laser beam. This 1200-nm absorption band was obtained by subtracting the spectrum taken before exposure to the laser from the spectrum taken during exposure to the laser. 
by the 325-nm laser, shown in Figure 5.6, peaks near $1200 \mathrm{~nm}$. This curve in Figure 5.6 is a difference spectrum (i.e., the absorption taken before the crystal was exposed to the laser has been subtracted from the absorption taken while the crystal was exposed to the laser). Additional data on the short wavelength side are not available because of the operational limits of our FTIR spectrometer. This 1200-nm absorption band was initially observed in Mg-doped stoichiometric $\mathrm{LiNbO}_{3}$ by Sweeney et al. [12]. These earlier investigators suggested that it was associated with a shallow electron trap, and we extend that assignment to specifically mean an electron trapped on a niobium ion at a regular lattice site. We found, in the present study, that the 1200-nm absorption band is sensitive to bleaching light, i.e., the responsible center was easily destroyed optically when experiments to determine its thermal stability, such as repeated absorption scans at various low temperatures, were attempted. Specifically, ultraviolet laser beams or $\mathrm{x}$ rays produce these absorbing centers while the infrared light present in the measuring beam of the FTIR spectrometer rapidly destroys the centers. The concentration of absorbing centers at any given time depends on the rates associated with these two competing mechanisms.

Low-temperature EPR spectra taken from one of our $1 \% \mathrm{Mg}$ stoichiometric $\mathrm{LiNbO}_{3}$ crystals are shown in Figures 5.7 and 5.8. These are complementary figures, differing only in the magnetic field regions covered. The crystal was irradiated at $77 \mathrm{~K}$ with $\mathrm{x}$ rays and rapidly transferred into the cold helium gas flowing through the microwave cavity, then the spectra in Figures 5.7 (a) and 5.8 (a) were taken at $20 \mathrm{~K}$ with the magnetic field along the c axis. Next, the conditions that produced the TL peak at $94 \mathrm{~K}$ (Figure 5.3) were replicated by warming the irradiated crystal to $110 \mathrm{~K}$ while in the microwave cavity, holding it there for 5 min, and finally returning to $20 \mathrm{~K}$ where the spectra in Figures 5.7 (b) and 5.8 (b) were taken (with the same spectrometer settings and the same direction of magnetic field). Similar EPR results, although with lower signal-to-noise ratios were observed when the $1 \% \mathrm{MgO} \mathrm{LiNbO}_{3}$ crystal was initially exposed to the $325-\mathrm{nm}$ or $355-\mathrm{nm}$ laser beams while being held in the 
microwave cavity at temperatures below $77 \mathrm{~K}$. It is important to note that we did not observe EPR signals from niobium antisite defects $\left(\mathrm{Nb}_{\mathrm{Li}}{ }^{4+}\right)$ in our stoichiometric samples after the low-temperature irradiations with $\mathrm{x}$ rays or the ultraviolet lasers. In contrast, congruent $\mathrm{LiNbO}_{3}$ crystals contain large concentrations of niobium ions that occupy lithium sites and provide the needed charge compensation for lithium vacancies introduced during growth; these $\mathrm{Nb}_{\mathrm{Li}}{ }^{5+}$ centers trap electrons during low-temperature x-ray irradiations and form paramagnetic $\mathrm{Nb}_{\mathrm{Li}}{ }^{4+}$ centers $[45,50]$.

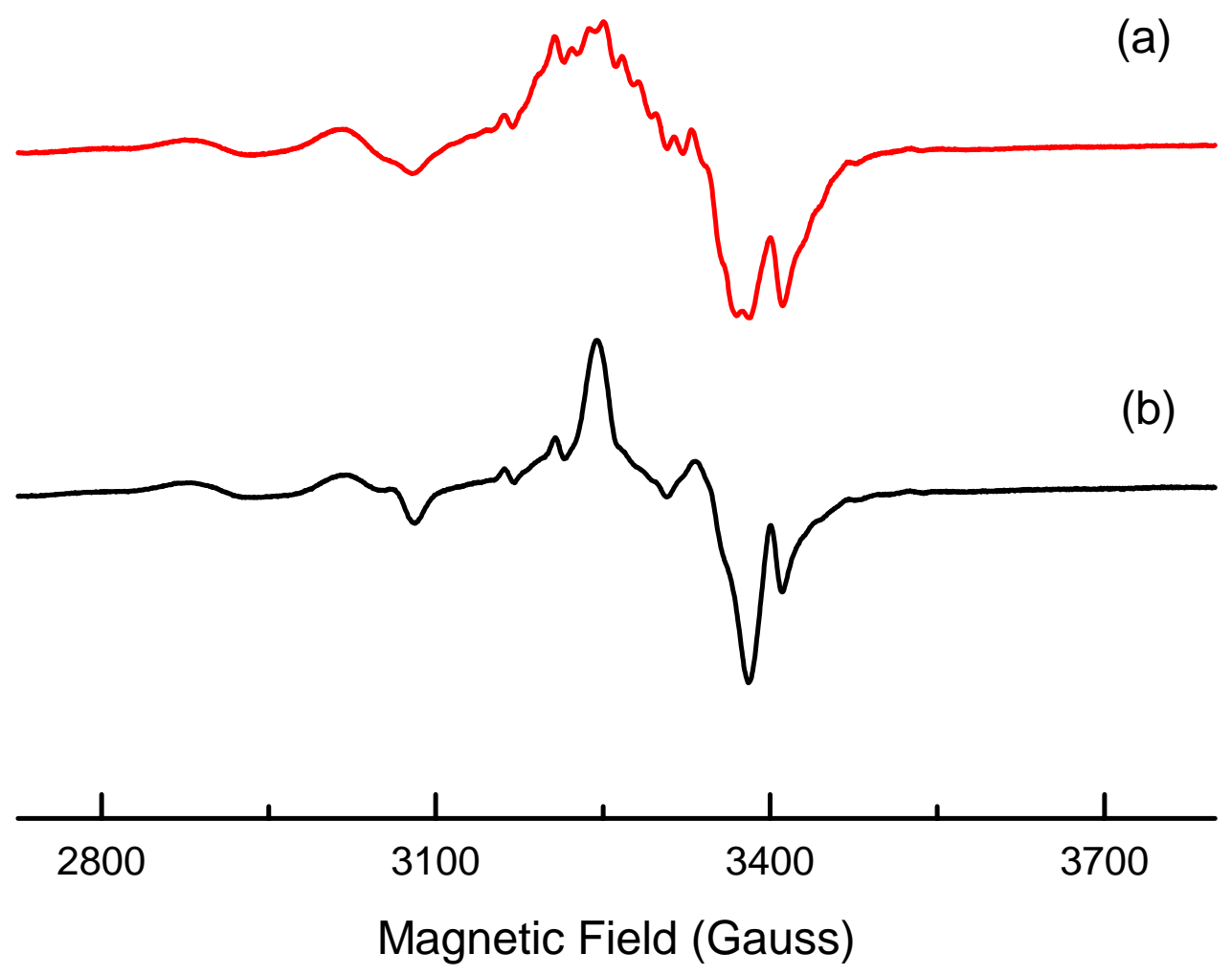

Figure 5.7. EPR data, taken at $20 \mathrm{~K}$ with the magnetic field along the $\mathrm{c}$ axis, showing the broad trapped-hole center associated with $\mathrm{Li}$ vacancies in a $1 \% \mathrm{Mg}$ stoichiometric $\mathrm{LiNbO}_{3}$ crystal. Trace (a) was taken after the crystal was irradiated with $\mathrm{x}$ rays at $77 \mathrm{~K}$ and trace (b) was taken after the crystal was held for five min at $110 \mathrm{~K}$. 
There are several paramagnetic point defects contributing to the EPR spectrum in Figure 5.7 (a). Because it represents the defect with the largest concentration, we draw attention to the broad underlying signal (approximately $150 \mathrm{G}$ wide and centered near 3300 G) covering the central portion of the spectrum. This broad EPR signal has a g value near 2.03 and has been assigned to a trapped-hole center, i.e., a hole trapped on an oxygen ion adjacent to a lithium vacancy $[45,50]$. In Figure 5.7 (b), after the crystal was warmed to 110 $\mathrm{K}$ and then returned to $20 \mathrm{~K}$, the broad trapped-hole signal has decreased to approximately $40 \%$ of its initial size. There are two unidentified lines in Figure 5.7 (b) that appeared as a result of holding the crystal briefly at $110 \mathrm{~K}$. They have distorted shapes, one pointing upward near $3245 \mathrm{G}$ and the other pointing downward near $3390 \mathrm{G}$, and they likely arise from a single defect. Since their integrated intensity (i.e., concentration) is considerably less than that of the broad trapped-hole center, we will not include these two lines in our discussion.

A very unusual EPR "feature" is present in Figure 5.8 (a). Instead of having a normal derivative shape, it only drops below the baseline with a minimum occurring near $4400 \mathrm{G}$. This feature is part of a broader EPR signal that was initially observed in Mg-doped stoichiometric $\mathrm{LiNbO}_{3}$ by Sweeney et al [12]. The entire spectrum extends from $2900 \mathrm{G}$ to $4400 \mathrm{G}$ when the magnetic field is along the c axis (see Fig. 3 in reference [12]), and was referred to as a "new electron trap" by these investigators. This broad EPR spectrum also has been observed by Zaritskii et al. [51] and referred to as a "thermal defect." Based on these two investigations, the presently accepted model of the center responsible for this unique spectrum is an electron trapped at a regular niobium site (i.e., a $\mathrm{Nb}_{\mathrm{Nb}}{ }^{4+}$ ) with perhaps a $\mathrm{Mg}_{\mathrm{Li}}{ }^{2+}$ ion nearby. Note that this center also gives rise to the $1200-\mathrm{nm}$ absorption band. We refer to this center as the "shallow trapped electron," and view it to a first approximation as a self-trapped electron while at the same time acknowledging that a $\mathrm{Mg}^{2+}$ ion may be in the near vicinity. In Figure 5.8 (b), taken after the crystal was warmed to $110 \mathrm{~K}$ and then 
returned to $20 \mathrm{~K}$, the EPR feature associated with the shallow trapped electron is no longer present.

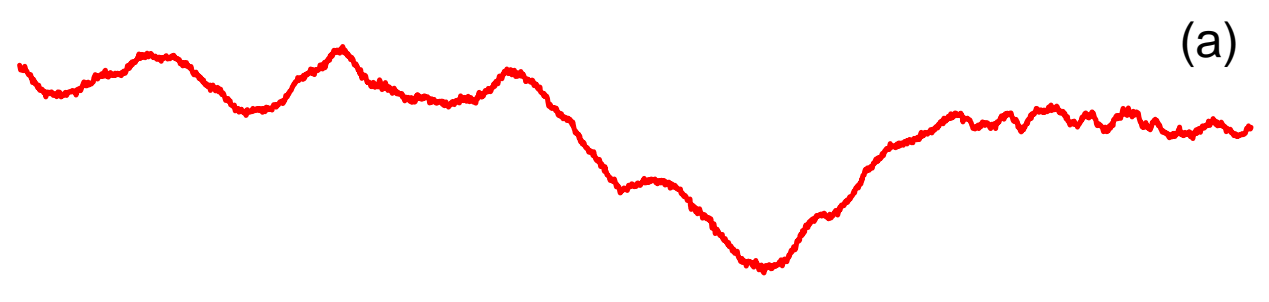

(b)
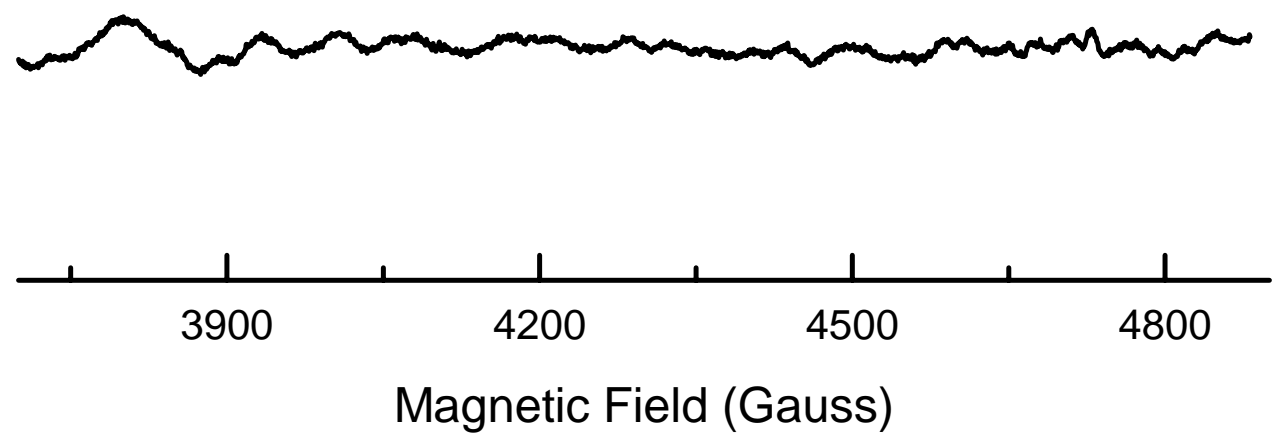

Figure 5.8. EPR data, taken at $20 \mathrm{~K}$ with the magnetic field along the c axis, showing the high-field portion of the spectrum associated with the shallow electron trap in $\mathrm{LiNbO}_{3}$. Trace (a) was taken after the crystal was irradiated with $\mathrm{x}$ rays at $77 \mathrm{~K}$ and trace (b) was taken after the crystal was held for five min at $110 \mathrm{~K}$.

The EPR signals associated with the hole center and the electron center in Figure 5.7 (a) and 5.8 (a) are not easily discerned. However, we have reproduced the spectra shown in these two figures numerous times and we are confident of their validity. There are three 
primary reasons why we do not observe larger and more distinct EPR signals from the trapped hole and the shallow electron centers. First, the sensitivity of the EPR spectrometer is reduced when monitoring broad signals (i.e., the minimum detectable number of spins increases significantly when the EPR line widths are broad). Second, luminescence is a more sensitive technique than EPR, so the number of electron and hole traps needed to produce an easily seen TL peak is less than the number required to produce an observable EPR signal. Third, there is significant interference (especially in Figure 5.7) from other low-concentration paramagnetic defects such as transition-ion impurities that distracts from the principal centers.

\subsubsection{Discussion and model}

Optical absorption in the near-infrared region and EPR measurements provided the key information needed to identify the electron and hole traps participating in the 94-K TL peak. The EPR results suggest that the usual trapped hole center, reported by earlier investigators $[12,50]$, is produced in a $1 \% \mathrm{Mg}$ stoichiometric crystal at $77 \mathrm{~K}$ by the $\mathrm{x}$ rays and ultraviolet lasers. We base this assignment on the observed linewidth of $150 \mathrm{G}$ and the $\mathrm{g}$ value of 2.03 (Figure 5.7 (a)). The model for this holelike center, although not absolutely established in the literature, is commonly accepted to be a hole trapped on an oxygen ion adjacent to a lithium vacancy [38]. Our EPR and optical absorption data suggest that a shallow electron center, reported by earlier investigators [12,51], is produced in a $1 \% \mathrm{Mg}$ stoichiometric crystal at $77 \mathrm{~K}$ by the $\mathrm{x}$ rays and ultraviolet lasers. This assignment is based on the presence of the 1200-nm absorption band and the broad downward EPR feature near $4400 \mathrm{G}$. The model for this shallow electron center is an electron trapped on a regular niobium with perhaps a $\mathrm{Mg}^{2+}$ ion nearby. We note that these shallow electron centers are not readily formed in congruent $\mathrm{LiNbO}_{3}$ crystals because of the presence of a very large concentration of niobium antisites defects $\left(\mathrm{Nb}_{\mathrm{Li}}{ }^{5+}\right)$. These antisite ions are closely spaced on average in the congruent crystals. Thus, if a niobium antisite defect is nearby, an electron 
will not remain localized on a regular niobium but will move the several lattice spaces to the deeper niobium antisite trap (because of the strong electrostatic attraction exerted by the highly charged antisite defect). The decreased probability of stabilizing a significant number of isolated shallow electron centers explains why the intensity of the blue emission is usually much smaller in congruent $\mathrm{LiNbO}_{3}$ crystals.

The warming step to $110 \mathrm{~K}$, illustrated in Figure 5.7 and 5.8, provides information about the mechanisms responsible for the observed thermoluminescence peak. When the temperature of the irradiated crystal was increased to $110 \mathrm{~K}$ (i.e., when replicating the TL experiment), approximately $60 \%$ of the hole centers disappeared and $100 \%$ of the shallow electron centers disappeared. These results suggest that the TL peak at $94 \mathrm{~K}$ is produced when the trapped electrons become unstable and move to the trapped hole centers. More specifically, the center that disappears completely is the center that becomes unstable and moves. Thus, we suggest that the radiative recombination occurs at or very near the hole site. Early investigators $[10,11,16]$ proposed that laser- and x-ray-induced luminescence from undoped $\mathrm{LiNbO}_{3}$ was a result of recombination at regular $\mathrm{NbO}_{6}$ units, and our present thermoluminescence data are not in significant disagreement with this suggestion. We envision the recombination process to occur as follows. The shallow electron centers become thermally unstable near $94 \mathrm{~K}$, the released electrons then move from niobium to niobium until they reach a niobium next to the oxygen with the trapped hole, and finally the hole and the electron recombine. In our model, the recombination takes place within an $\mathrm{NbO}_{6}$ unit containing the oxygen ion where the hole was initially trapped. This is not a regular $\mathrm{NbO}_{6}$ unit, but one that is slightly perturbed by a neighboring lithium vacancy (i.e., the lithium vacancy that initially stabilized the hole). When visualizing our recombination model, it is important to recall that each oxygen ion in the $\mathrm{LiNbO}_{3}$ lattice has four cation neighbors, two lithium and two niobiums.

A final point refers to the mechanisms involved in initially producing the trapped 
holes and trapped electrons that contribute to the 94-K TL peak. A direct band-to-band ionization process is expected in the case of the $60-\mathrm{kV}$ x rays. In contrast, we note that our laser wavelengths (325 and $355 \mathrm{~nm}$ ) are well below the fundamental band edge of the crystal at $77 \mathrm{~K}$ (see Figure 5.1). For photons at these wavelengths to form separated electrons and holes (that recombine on warming), we suggest that the broad charge-transfer absorption band of $\mathrm{Fe}^{3+}$ ions, peaking near $320 \mathrm{~nm}$, must be involved. The precise mechanism is unknown, but we can envision the following scenario. Absorption of a photon in the charge transfer band will result in the transient formation of an $\mathrm{Fe}^{2+}$ and an adjacent $\mathrm{O}^{-}$ion, as an electron is transferred temporarily from the oxygen to the iron. This electron and hole may simply recombine nonradiatively at the original $\mathrm{Fe}-\mathrm{O}$ site and restore the original charge configuration. It is also possible that the electron and the hole will separate (since the newly formed $\mathrm{Fe}^{2+}$ and the hole both have an effective positive charge relative to the regular lattice). This electrostatic repulsion force on the hole may cause it to move away from the $\mathrm{Fe}^{2+}$, and thus become free to migrate through the lattice until it finds a stabilizing entity such as lithium vacancy. A second photon could then "move" the electron away from the remaining $\mathrm{Fe}^{2+}$ ion. This would occur in the same way that photorefractive gratings are written in $\mathrm{Fe}-$ doped $\mathrm{LiNbO}_{3}$ (i.e., electrons are "moved," in an intervalence transfer [47], from $\mathrm{Fe}^{2+}$ ions to a neighboring niobium ion by wavelengths that fall within the very broad 490 -nm optical absorption band associated with the $\mathrm{Fe}^{2+}$ ions). This absorption of the second photon by the $\mathrm{Fe}^{2+}$ ion restores the initial $\mathrm{Fe}^{3+}$ ion and, at the same time, forms a shallow trapped electron center at one of the regular niobium sites well removed from the initial $\mathrm{Fe}^{3+}$ ion. Although this scenario to produce separated electrons and holes with below-band-gap light involves the sequential absorption of two photons, the corresponding formation rate is expected to depend linearly on light intensity. A linear dependence results from the long lifetimes, at $77 \mathrm{~K}$, of the trapped hole and the $\mathrm{Fe}^{2+}$ ion formed in the first stage of the process.

There may be other variations of the scenario we have just described that involve a 
more direct transfer of an electron into the conduction band by below-band-gap light. In particular, it is possible that the absorption of a photon in the charge transfer band of the $\mathrm{Fe}^{3+}$ ions results in the transient formation of an $\mathrm{Fe}^{2+}$ (in its excited state), with an adjacent $\mathrm{O}^{-}$ion, and that thermal energy then moves the excited-state electron from the iron to the conduction band. This leaves the hole on the oxygen free to migrate away to a trapping entity such a lithium vacancy.

\section{2. $\mathrm{LiTaO}_{3}$}

\subsubsection{TL, EPR, and optical absorption results}

In the present section, we describe thermoluminescence, optical absorption, and electron paramagnetic results obtained from stoichiometric $\mathrm{LiTaO}_{3}$. The crystals were grown from a congruent melt and then were made stoichiometric by a post-growth vapor-transportequilibration (VTE) treatment. In our TL experiments, a sample was initially exposed to $\mathrm{x}$ rays or an ultraviolet laser beam $(266,325$, or $355 \mathrm{~nm})$ while being held at $77 \mathrm{~K}$. A subsequent rapid warming produced overlapping TL "glow" peaks at 94 and $98 \mathrm{~K}$, each with a maximum spectral output near $350 \mathrm{~nm}$. Optical absorption in the near-infrared and EPR data, taken after similar irradiations, were used to identify the specific electron and hole centers participating in the radiative recombination process.

First, I will present optical absorption data showing the absorption band edge. The data in Figure 5.9 were taken at $77 \mathrm{~K}$ and room temperature using unpolarized light propagating along the $\mathrm{c}$ axis. These data show the shift in the band edge from $262 \mathrm{~nm}$ to 248 $\mathrm{nm}$ as the temperature is lowered. There is a near-edge absorption band in these spectra that is attributed to a charge-transfer transition of $\mathrm{Fe}^{3+}$ ions (i.e., a transition in which an electron moves from an adjacent oxygen ion to the Fe ion) [47,48]. It peaks at approximately $300 \mathrm{~nm}$ and does not shift appreciably as the temperature is lowered. A possible second charge 
transfer band, associated with $\mathrm{Fe}^{3+}$ or another transition-metal-ion impurity is emerging near $255 \mathrm{~nm}$ in the absorption spectrum taken at $77 \mathrm{~K}$. We note that our VTE-treated $\mathrm{LiTaO}_{3}$ crystals exhibited sharp EPR lines from traces levels of $\mathrm{Fe}^{3+}$ and $\mathrm{Co}^{2+}$ impurity ions.

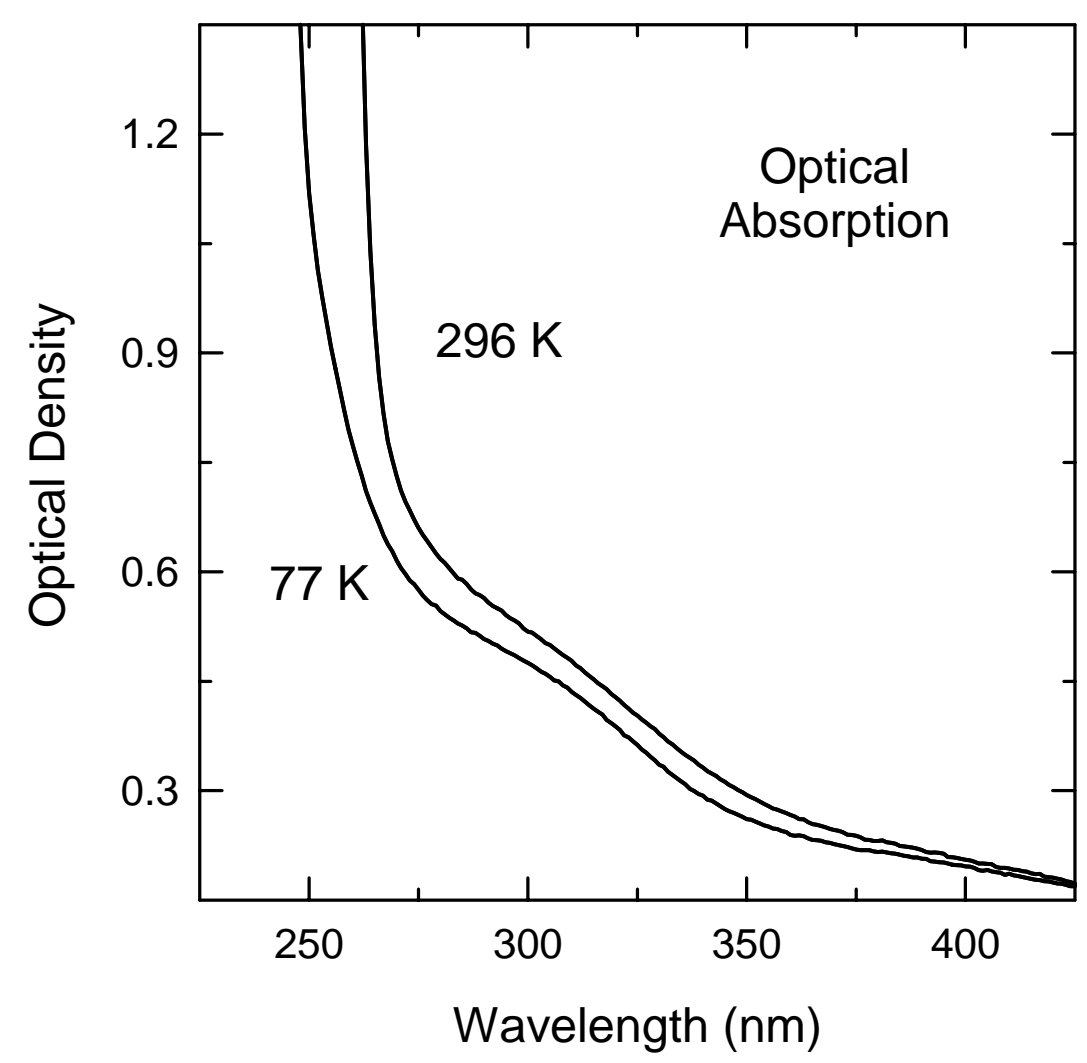

Figure 5.9. Optical absorption from a VTE-treated $\mathrm{LiTaO}_{3}$ crystal. These data were taken at room temperature and at $77 \mathrm{~K}$ with unpolarized light propagating along the $\mathrm{c}$ axis. The absorption band near $300 \mathrm{~nm}$ is attributed to trace amounts of $\mathrm{Fe}^{3+}$ ions.

Thermoluminescence results from a VTE-treated $\mathrm{LiTaO}_{3}$ crystal are shown in Figure 5.10. These data are presented in a three-dimensional plot with temperature, wavelength, and 
intensity as the axes. Prior to the warming depicted in Figure 5.10, the sample was exposed to the pulsed $355-\mathrm{nm}$ laser beam while being held at $77 \mathrm{~K}$. Then, a rapid increase in temperature produced the TL. The rate of heating was approximately $9.6 \mathrm{~K} / \mathrm{sec}$, and this allowed the spectrograph and CCD camera to obtain a complete wavelength dependence of the emission every $1.0 \mathrm{~K}$.

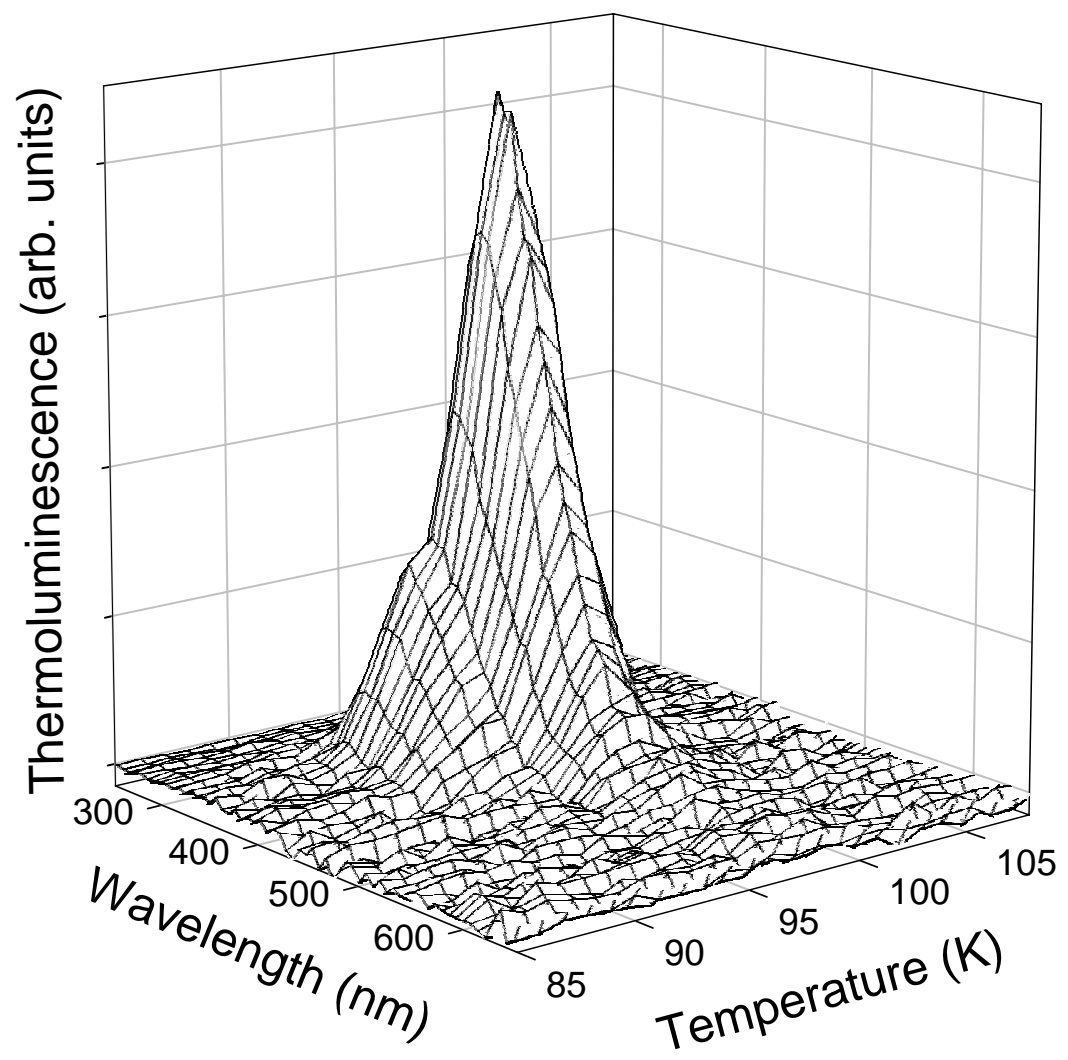

Figure 5.10. Three-dimensional view of the TL data from a VTE-treated $\mathrm{LiTaO}_{3}$ crystal that had been irradiated at $77 \mathrm{~K}$ with a pulsed $355-\mathrm{nm}$ laser. The intensity of the emission is plotted as a function of both temperature and wavelength. 
Figures 5.11 and 5.12 contain cross-sectional views of the data in Figure 5.10. The temperature dependence, shown in Figure 5.11, was measured at the wavelength corresponding to the peak of the emission. There are two very closely spaced peaks in this TL spectrum, a weak emission near $94 \mathrm{~K}$ and a more intense peak near $98 \mathrm{~K}$. We repeated

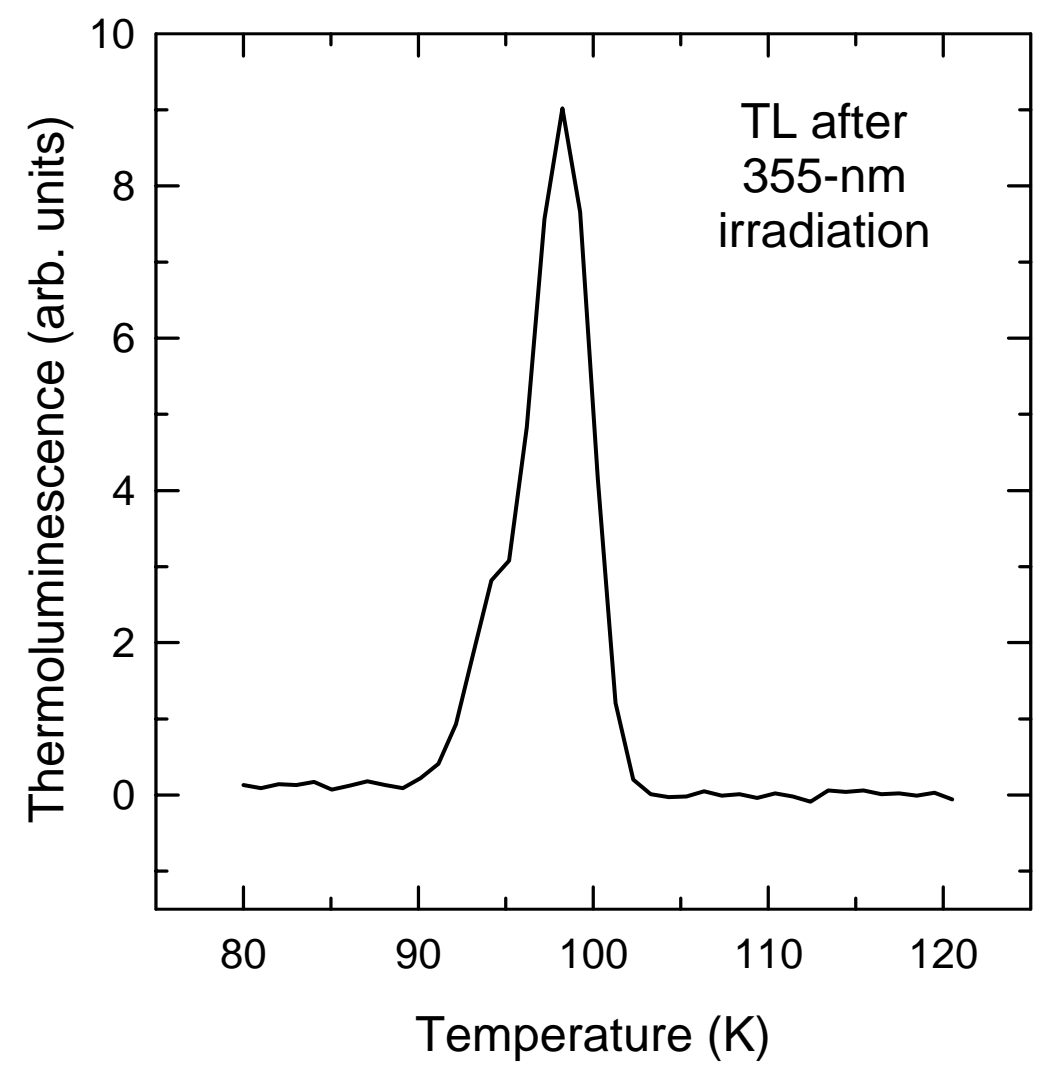

Figure 5.11. A cross-section view (taken from Figure 5.10) showing the temperature dependence of the TL from a VTE-treated $\mathrm{LiTaO}_{3}$ crystal. These data correspond to a wavelength of $350 \mathrm{~nm}$ (i.e., the maximum in the emission). Two overlapping TL peaks are observed, one near $94 \mathrm{~K}$ and one near $98 \mathrm{~K}$.

this experiment numerous times to verify the existence of both TL peaks. The wavelength dependence shown in Figure 5.12 represents the emission observed at $98 \mathrm{~K}$ (the same 
wavelength dependence also was observed at $94 \mathrm{~K}$ ). These data in Figure 5.12 have been corrected for system response, and they show that the peak in the emission occurs near 350 $\mathrm{nm}$. In addition to the pulsed 355-nm laser beam, three other excitation sources $(60-\mathrm{kV} \mathrm{x}$ rays, a pulsed 266-nm laser beam, and a cw 325-nm laser beam) were also used to produce the TL in the VTE-treated $\mathrm{LiTaO}_{3}$ crystals. The results obtained using these latter excitation sources were identical to the results shown in Figures 5.10, 5.11, and 5.12. In contrast, we did not observe any emission from our congruent $\mathrm{LiTaO}_{3}$ crystals, even though the same experimental procedures were followed and the same excitation sources were used.

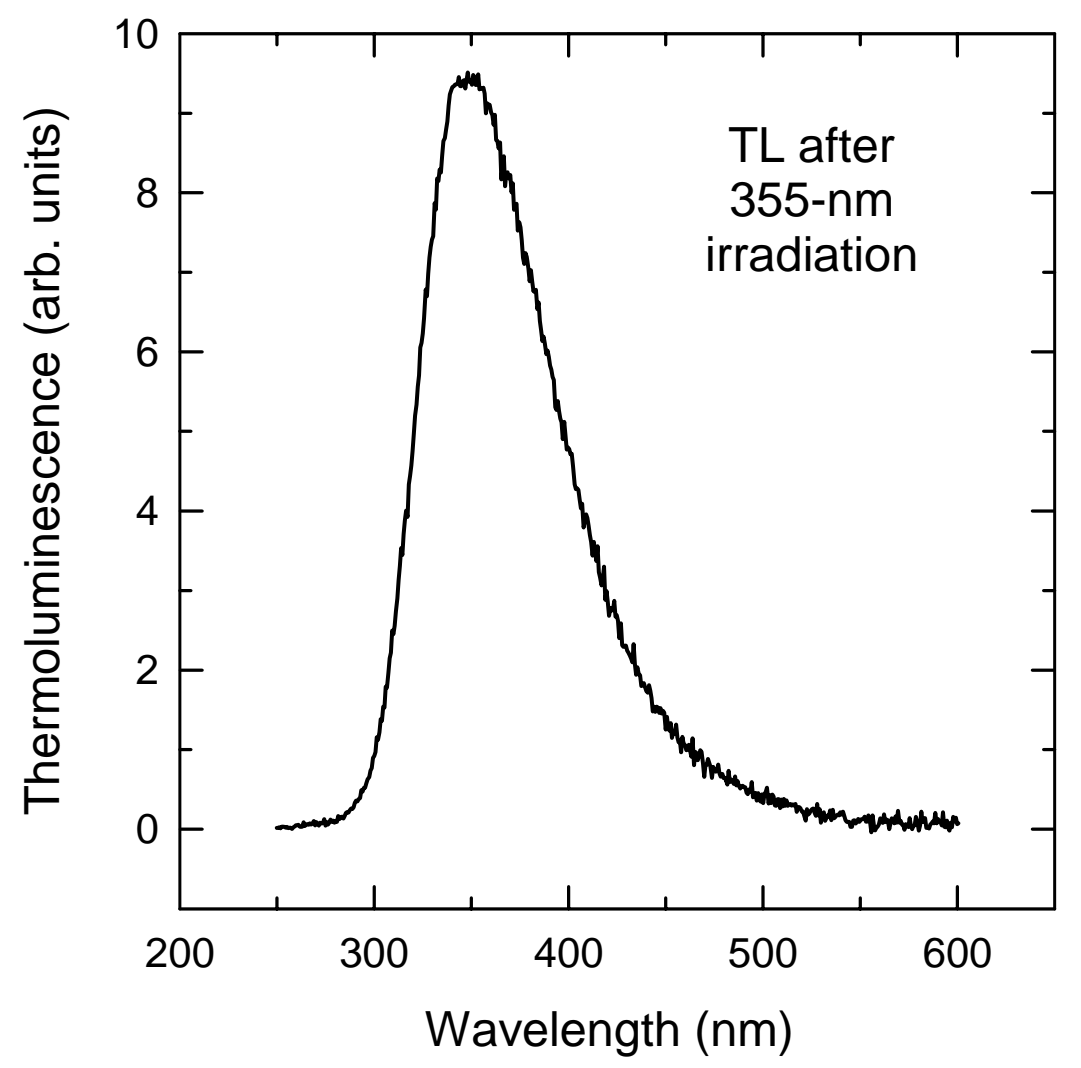

Figure 5.12. A cross-section view (taken from Figure 5.10) showing the wavelength dependence of the TL from a VTE-treated $\mathrm{LiTaO}_{3}$ crystal. These data correspond to a temperature of $98 \mathrm{~K}$ (i.e., the maximum of the emission). The TL peak occurs near $350 \mathrm{~nm}$. 
Separate optical absorption and EPR experiments were performed to help identify the electron and hole centers contributing to the TL peaks in Figure 5.10. Optical absorption data in the near infrared were obtained at $10 \mathrm{~K}$ before and during the exposure of a VTEtreated $\mathrm{LiTaO}_{3}$ crystal to a $325-\mathrm{nm}$ laser beam. This ultraviolet laser excitation produced a broad absorption band peaking near $1600 \mathrm{~nm}$, as shown in Figure 5.13. The curve presented

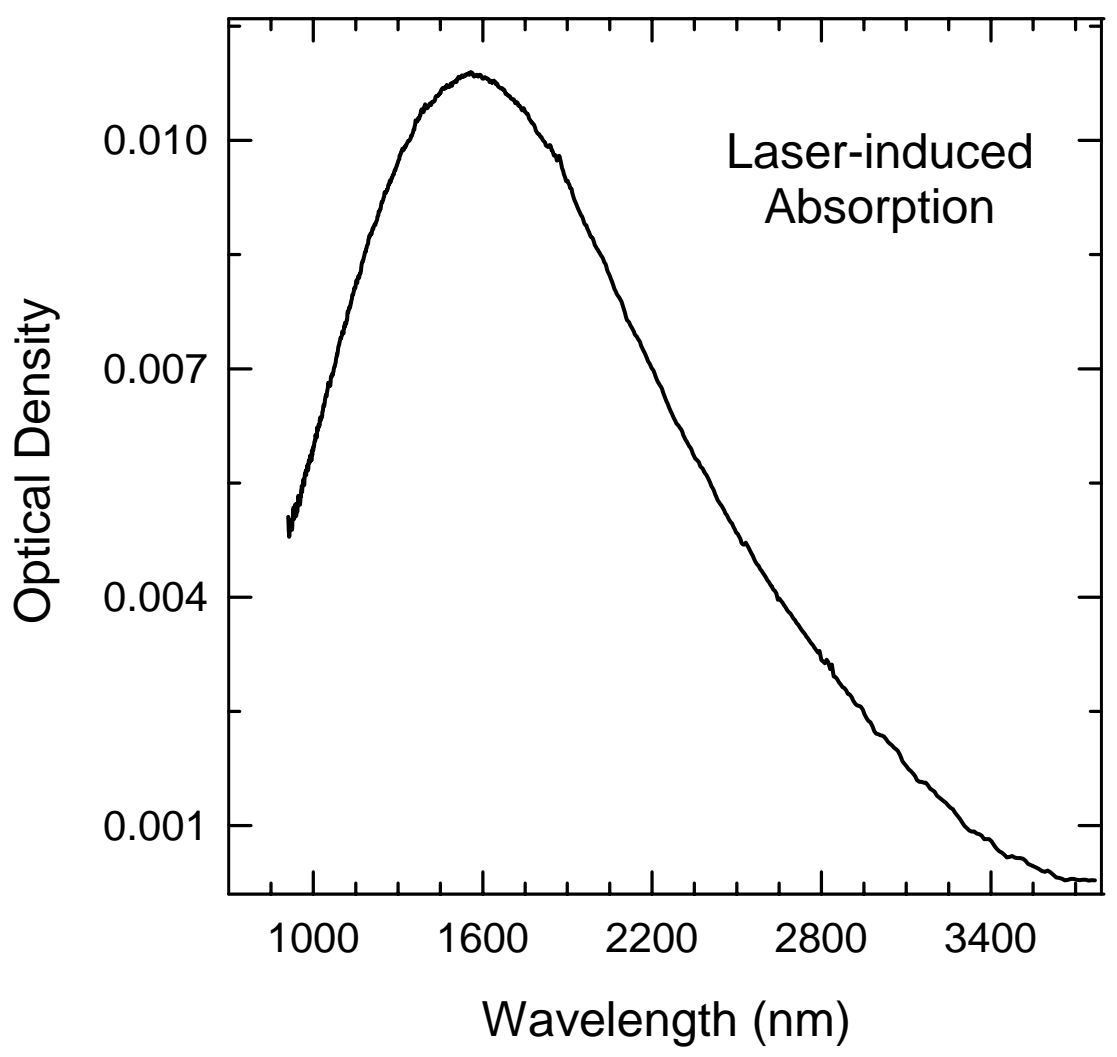

Figure 5.13. Optical absorption from a shallow electron trap, produced at $10 \mathrm{~K}$ by a 325-nm laser beam. This 1600-nm absorption band was obtained by subtracting the spectrum taken before exposure to the laser from the spectrum taken during exposure to the laser. 
in this figure is a "difference" spectrum (i.e., absorption data taken before the crystal was exposed to the laser have been subtracted from absorption data taken while the crystal was exposed to the laser). This $1600-\mathrm{nm}$ absorption band, optically induced at low temperature, has not been previously reported in $\mathrm{LiTaO}_{3}$ crystals. When considering an assignment for this band, we note its similarity to the infrared absorption band peaking near $1200 \mathrm{~nm}$ in stoichiometric $\mathrm{LiNbO}_{3}$ crystals [12,53]. In the former material, the infrared absorption band is attributed to a shallow electron trap, i.e., an electron trapped on a regular substitutional niobium ion. By analogy, we suggest that the 1600-nm absorption band formed at low temperature in VTE-treated $\mathrm{LiTaO}_{3}$ is also a shallow electron trap, with the electron localized on a regular substitutional tantalum ion (i.e., a $\mathrm{Ta}_{\mathrm{Ta}}{ }^{4+}$ center). Unfortunately, this 1600-nm absorption band is sensitive to infrared bleaching light and is easily destroyed optically when experiments to determine its thermal stability, such as repeated absorption scans at various low temperatures, are attempted.

The series of low-temperature EPR spectra shown in Figure 5.14 were obtained from a VTE-treated $\mathrm{LiTaO}_{3}$ crystal. These data were taken at $40 \mathrm{~K}$ with the magnetic field parallel to the c axis. Trace (a) in Figure 5.14 is from the as-treated crystal, prior to irradiation. The more intense EPR signal located near $3380 \mathrm{G}$ is one of several lines associated with the $\mathrm{Fe}^{3+}$ ions that substitute for $\mathrm{Li}^{+}$ions [54]. The crystal then was irradiated at $77 \mathrm{~K}$ with $\mathrm{x}$ rays and rapidly transferred into the cold helium gas $(\approx 40 \mathrm{~K})$ flowing through the microwave cavity. Trace (b) was taken after the irradiation, but before an annealing step. The $\mathrm{Fe}^{3+}$ signal has decreased significantly (by a factor of more than three) as a result of the 77-K irradiation, and a three-line spectrum has appeared. This latter spectrum has been reported in several earlier studies $[31,36]$ and is assigned to a hole center, where the hole is either self-trapped on one or more oxygen ions in an otherwise perfect region of the crystal or trapped on an oxygen ion next to a lithium vacancy.

Next, the conditions that produced the TL peaks at $94 \mathrm{~K}$ and $98 \mathrm{~K}$ (see Figure 5.11) 
were replicated by warming the irradiated crystal to $100 \mathrm{~K}$ while in the microwave cavity, holding it there for $5 \mathrm{~min}$, and then finally returning to $40 \mathrm{~K}$ where the spectrum in trace (c) of Figure 5.14 was taken (with the same spectrometer settings and the same orientation of magnetic field). This $100-\mathrm{K}$ anneal removed the three-line trapped hole center and increased the concentration of $\mathrm{Fe}^{3+}$ ions by approximately $50 \%$. At the same time, a new four-line hole center has emerged, as illustrated by the lower stick diagram for trace (c) in Figure 5.14.

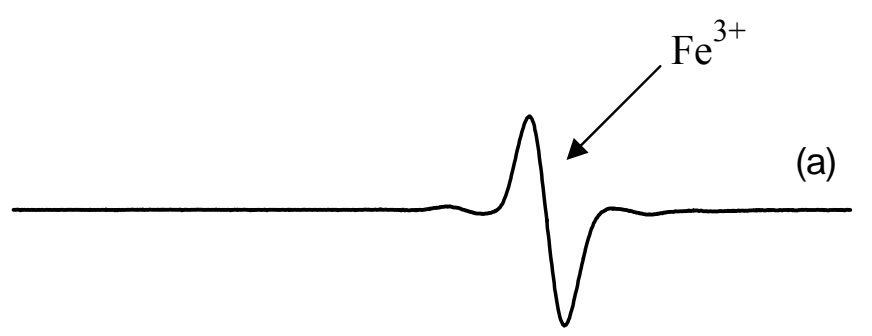

(b)

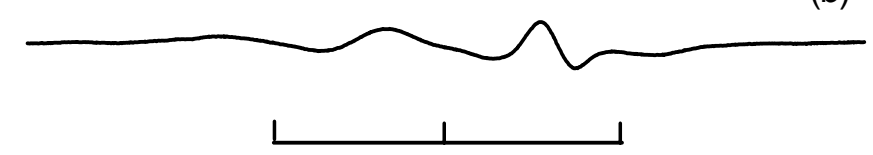

(c)
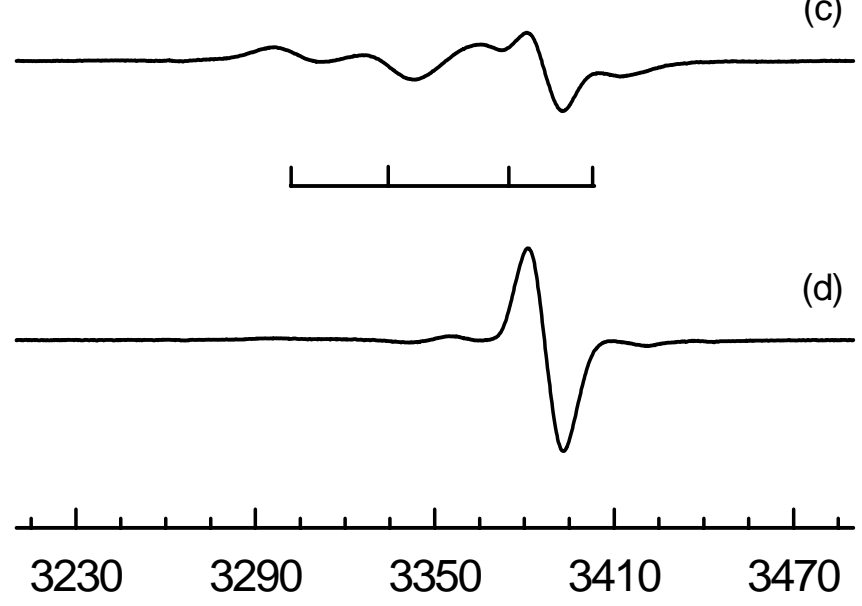

Magnetic Field (Gauss)

Figure 5.14. EPR spectra from a VTE-treated $\mathrm{LiTaO}_{3}$ crystal taken (a) before irradiation, (b) after exposure to $\mathrm{x}$ rays at $77 \mathrm{~K}$, (c) after a five-min anneal at $100 \mathrm{~K}$, and (d) after a five-min anneal at $130 \mathrm{~K}$. The measurement temperature was $40 \mathrm{~K}$ and the magnetic field was parallel to the $\mathrm{c}$ axis. 
This four-line EPR spectrum has not been previously reported. Finally, in a second and final anneal, the sample was warmed to $130 \mathrm{~K}$ while in the microwave cavity, held for $5 \mathrm{~min}$, and then returned to $40 \mathrm{~K}$ where the spectrum in trace (d) of Figure 5.14 was taken. During this anneal, the four-line hole center disappeared completely and the concentration of $\mathrm{Fe}^{3+}$ ions returned to the pre-irradiation value, as shown by comparing trace (d) with trace (a). Similar EPR results although with lower signal-to-noise ratio, were observed when a VTE-treated $\mathrm{LiTaO}_{3}$ crystal was initially exposed to a $325-\mathrm{nm}$ laser beam while being held in the microwave cavity at temperatures below $77 \mathrm{~K}$. Experiments were not performed to determine if the 266 and $355 \mathrm{~nm}$ laser beams would produce these EPR results since it was difficult for us to transfer these beams to the microwave cavity.

\subsubsection{Discussion and model}

The thermally stimulated luminescence described here has only been observed in undoped $\mathrm{LiTaO}_{3}$ crystals that were congruently grown and then VTE-treated. When a crystal was cut from the same congruently grown boule, but not VTE-treated, then the emission was not seen. It is generally known (in analogy with $\mathrm{LiNbO}_{3}$ ) that congruently grown $\mathrm{LiTaO}_{3}$ crystals contain large numbers of lithium vacancies, with charge compensation provided by tantalum antisite defects (i.e., tantalum ions substituting for lithium ions). During the postgrowth VTE treatment, lithium ions enter the crystal and eliminate nearly all the of these lithium vacancies. The tantalum ions that initially occupy lithium sites are then no longer needed for charge compensation and they diffuse to the surface of the crystal. This process leaves the $\mathrm{LiTaO}_{3}$ crystal in a form that is very nearly stoichiometric. We suggest that the occurrence of radiative recombination (i.e., emission of light when electrons and holes recombine) at low temperatures in the VTE-treated $\mathrm{LiTaO}_{3}$ crystals directly correlates with the removal of these lithium vacancies and the tantalum antisite defects. Removal of the 
tantalum antisite defects is especially important because they were providing an efficient nonradiative recombination site for electrons and holes in the congruent $\mathrm{LiTaO}_{3}$ crystals.

Our EPR and optical absorption data provide information about the electron and hole traps that contribute to the TL peaks observed between 90 and $110 \mathrm{~K}$ in $\mathrm{LiTaO}_{3}$. Referring to Figure 5.14, we see in trace (b) that an irradiation at $77 \mathrm{~K}$ with $\mathrm{x}$ rays produces a "threeline" EPR spectrum representing a trapped-hole center and also decreases the intensity of the $\mathrm{Fe}^{3+}$ EPR line (indicating that the $\mathrm{Fe}^{3+}$ ions trap an electron and become $\mathrm{Fe}^{2+}$ ions). In addition, our optical absorption results in Figure 5.13 show that ionizing radiation at low temperature forms a shallow electron center when an electron is trapped on a regular tantalum ion (i.e., the $\mathrm{Ta}_{\mathrm{Ta}}{ }^{4+}$ center). This suggests that one hole center and two electron centers are formed during the 77-K irradiation, and that the number of hole centers produced is equal to the combined number of electron centers.

Next, we consider the behavior of these defects when the crystal is warmed to $100 \mathrm{~K}$, an annealing step that duplicates the conditions that caused the TL peaks. Trace (c) in Figure 5.14 shows that the three-line hole center is no longer observable and the $\mathrm{Fe}^{3+}$ has only partially recovered. This suggests that the three-line trapped-hole centers become thermally unstable below $100 \mathrm{~K}$, thus allowing these holes to migrate to other defects. There are three destinations for these holes. Some of the holes will move to $\mathrm{Ta}_{\mathrm{Ta}}{ }^{4+}$ defects and others will move to $\mathrm{Fe}^{2+}$ ions, recombining with the trapped electrons in each case. At the same time, a portion of the holes will move to "deeper" trapping sites in the crystal and form a new more stable hole center. Verification that the latter two cases occur is provided by trace (c) in Figure 5.14 when the $\mathrm{Fe}^{3+}$ signal partially recovers and a new four-line hole center appears. We expect that recombination at the $\mathrm{Ta}_{\mathrm{Ta}}{ }^{4+}$ centers will be radiative, and that recombination at the $\mathrm{Fe}^{2+}$ ions will be nonradiative. From this analysis, we conclude that the primary mechanism leading to the observed TL peaks in $\mathrm{LiTaO}_{3}$ is the release of holes from the threeline center and their recombination with electrons that had been "self-trapped" in the form of 
$\mathrm{Ta}_{\mathrm{Ta}}{ }^{4+}$ centers. The appearance of the two TL peaks, at 94 and $98 \mathrm{~K}$, may result from one of the following two possibilities. First, the three-line hole center has relatively broad EPR lines and could represent two very similar, yet distinct, hole centers that would thermally release their holes at slightly different temperatures. Second, we do not know the decay temperature for the shallow tantalum electron center, but we believe it also may be in the mid-90 $\mathrm{K}$ region, by analogy with $\mathrm{LiNbO}_{3}$ [53]. If this is the case, then within a small temperature range, we would have holes moving to recombine at sites where electrons are trapped and also electrons moving to recombine at sites where holes are trapped (i.e., both the three-line hole centers and the $\mathrm{Ta}_{\mathrm{Ta}}{ }^{4+}$ centers may be radiative recombination sites). In each event, the recombination would take place in $\mathrm{TaO}_{6}$ units, and would agree with the observation that the 94 and $98 \mathrm{~K}$ TL peaks have the same spectral output, peaking near $350 \mathrm{~nm}$.

Although reported in earlier studies [31,36], a definitive model has not yet been established for the hole center represented by the three-line EPR spectrum in trace (b) of Figure 5.14. Plausible models in which the hole is localized on an oxygen ion, either selftrapped as a result of lattice relaxation or stabilized by an adjacent entity such as a lithium vacancy, were suggested in the previous section. Because the three lines in the c-axis EPR spectrum have 1:2:1 intensity ratios, it was initially thought that equal hyperfine interactions with two $100 \%$ abundant $\mathrm{I}=1 / 2$ nuclei such as hydrogen were responsible for the structure. However, the work of Gonzalez et al. [36] has cast considerable doubt on that possibility. We also note that at the present time there is no definitive model for the hole center represented by the four-line EPR spectrum in trace (c) of Figure 5.14. 


\section{CHAPTER 6}

\section{EXPERIMENTAL RESULTS FROM KD $\mathrm{PO}_{4}$}

In this chapter, we describe the results of a combined EPR and optical absorption study of point defects produced in an undoped DKDP crystal at $77 \mathrm{~K}$ with ionizing radiation. By monitoring all the defect spectra immediately after the x-ray irradiation and then again at various steps in a thermal anneal from $77 \mathrm{~K}$ to near room temperature, we have established a direct correlation between specific centers and optical absorption bands. Previous studies $[55,56,63]$ used either EPR or optical techniques and, thus, did not provide correlated data. In addition to the correlation of optical absorption bands with EPR signals, the present study provides information about the relative stability of the hole centers and electron centers as the temperature increases and specifically shows the conversions that occur when one defect becomes unstable and another trapping site becomes occupied as the hole or electron moves through the lattice.

\subsection{Optical absorption and EPR results}

The undoped DKDP crystals used in the present investigation were grown at Lawrence Livermore National Laboratory. Two samples, one for optical absorption measurements and the other for EPR measurements, were cut from adjacent positions in the large boule. The optical sample was a plate oriented perpendicular to the $\mathrm{c}$ axis with dimensions of $4 \mathrm{x} 12 \mathrm{x}$ $1.3 \mathrm{~mm}^{3}$. The EPR sample was rectangular with dimensions of $6 \times 3 \times 2.5 \mathrm{~mm}^{3}$. Optical absorption data were taken at temperatures between 40 and $300 \mathrm{~K}$ with a Cary 14 spectrophotometer refurbished by OLIS (Bogart, GA). EPR data were obtained near $40 \mathrm{~K}$ using a Bruker ESP-300 spectrometer. Helium-flow systems from Oxford Instruments were 
used to control the sample temperature during the optical absorption and EPR measurements.

Point defects were created in the DKDP samples by immersing the crystals directly in liquid nitrogen (i.e., $77 \mathrm{~K})$ and then irradiating with $\mathrm{x}$ rays $(60 \mathrm{kV}, 30 \mathrm{~mA})$ for $30 \mathrm{~min}$. After turning off the $\mathrm{x}$ ray tube, a sample was transferred into the stream of cold helium gas (at 40 $\mathrm{K}$ or lower) within 2 min with very little intervening warming. This effectively "freezes-in" the defects at these low temperatures, and they can then be systematically studied without fear of decay until a monitored sequence of annealing steps commences.

An initial survey of our as-grown DKDP crystals showed a very weak absorption band peaking near $270 \mathrm{~nm}$ (previously assigned [69] to trace amounts of $\mathrm{Fe}^{3+}$ impurities) and no EPR spectra. After the crystal was irradiated at $77 \mathrm{~K}$ with $\mathrm{x}$ rays, large optical absorption bands and large EPR signals were present.

Figure 6.1 shows the optical absorption spectra from a DKDP crystal before and after an irradiation at $77 \mathrm{~K}$ with $\mathrm{x}$ rays. These data were acquired using unpolarized light propagating along the $\mathrm{c}$ axis of the crystal. Trace a was taken at $40 \mathrm{~K}$ before the exposure to the $\mathrm{x}$ rays, while traces $\mathrm{b}, \mathrm{c}, \mathrm{d}, \mathrm{e}, \mathrm{f}$, and $\mathrm{g}$ were taken after the irradiation at 80, 100, 120, 140, 180 , and $200 \mathrm{~K}$, respectively. Although not shown, additional spectra were taken at 160, 220, 240,260 , and $300 \mathrm{~K}$. In this series of sequential measurements, it took five min to record one spectrum and another five min to raise the temperature to the next level. Thus, approximately ten min elapsed between the starting time to collect each spectrum.

The $\mathrm{x}$ rays introduced significant optical absorption in DKDP with overlapping bands covering the entire wavelength range from below $700 \mathrm{~nm}$ to above $200 \mathrm{~nm}$ (see trace $\mathrm{b}$ in Figure 6.1). Distinct peaks occur in our data at 230, 390, and $550 \mathrm{~nm}$.

Warming the DKDP crystal to $100 \mathrm{~K}$, after the initial irradiation at $77 \mathrm{~K}$, results in a small decrease in each of the three primary absorption bands (as illustrated in trace c of Figure 6.1). A much larger decrease in all three bands occurs when the crystal is warmed to $120 \mathrm{~K}$ (see trace $\mathrm{d}$ in Figure 6.1). Following the 120-K anneal step, the two bands peaking at 
390 and $550 \mathrm{~nm}$ are still observable, but greatly reduced in intensity and barely resolved.

The $230-\mathrm{nm}$ band is also significantly smaller in intensity after the $120-\mathrm{K}$ anneal step.

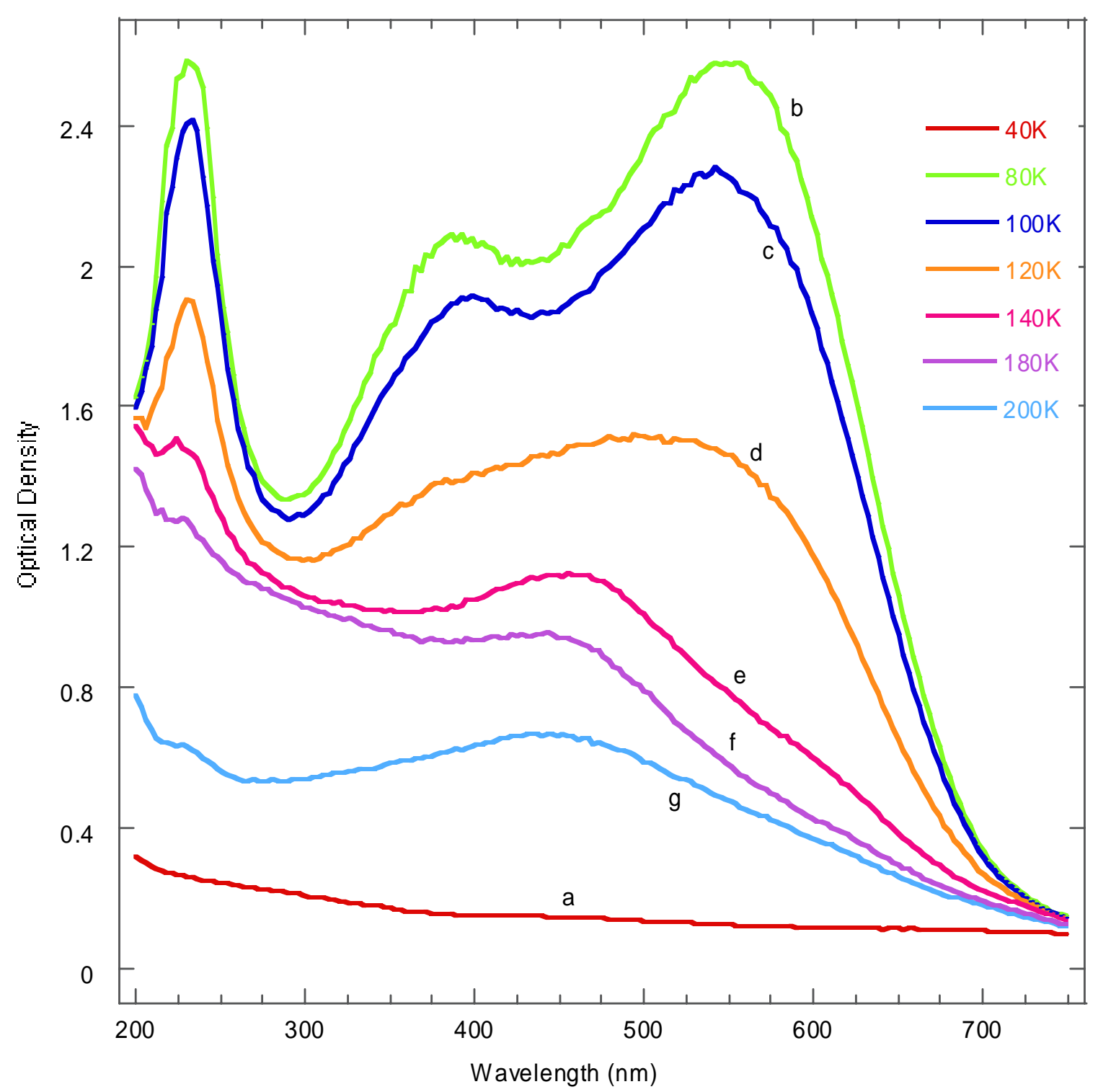

Figure 6.1. Optical absorption spectra from radiation-induced point defects in DKDP. Trace a was taken at $40 \mathrm{~K}$ from the unirradiated crystal. Then the crystal was irradiated at $77 \mathrm{~K}$ with $\mathrm{x}$ rays, and traces $\mathrm{b}, \mathrm{c}, \mathrm{d}$, e, f, and $\mathrm{g}$ were subsequently taken at $80 \mathrm{~K}, 100,120,140,180$, and $200 \mathrm{~K}$, respectively. 
Warming to $140 \mathrm{~K}$ essentially eliminates the two bands at 390 and $550 \mathrm{~nm}$ (i.e., they are not observed in trace e in Figure 6.1) and greatly reduces the 230-nm band. A broad band peaking near $450 \mathrm{~nm}$ emerges during the $140-\mathrm{K}$ anneal step. The absorption in the visible and ultraviolet is further reduced when the crystal is warmed to $180 \mathrm{~K}$ (trace $\mathrm{f}$ in Figure 6.1). After the annealing step at $200 \mathrm{~K}$, the absorption bands at 390 and $550 \mathrm{~nm}$ are greatly reduced but the band at $450 \mathrm{~nm}$ can still be easily observed. Although the data are not shown in Figure 6.1, continuing to warm the crystal to room temperature results in the elimination of nearly all of the radiation-induced absorption in the visible region (700 to $400 \mathrm{~nm}$ ) and leaves only a small amount of absorption in the ultraviolet region. This remaining absorption gradually increases from 400 to $200 \mathrm{~nm}$, without a significant structure, and is likely due to small concentrations of oxygen-vacancy centers and silicon-hole centers that survive to room temperature.

The initial three optical absorption bands, at 230, 390, and $550 \mathrm{~nm}$, formed right after the $\mathrm{x}$ ray irradiation of the DKDP crystal and before other bands form, can be distinguished in Figure 6.2. This spectrum was obtained by subtracting the optical absorption spectrum at $120 \mathrm{~K}$ from the spectrum taken at $80 \mathrm{~K}$ (i.e., trace $\mathrm{d}$ from trace $\mathrm{b}$, in Figure 6.1). The two broad band at 390 and $550 \mathrm{~nm}$ have been previously observed by Dieguez et al. $[63,64]$ in KDP and DKDP. These investigators tentatively suggested that the two bands may be associated with the self-trapped holes (i.e., the $\left[\mathrm{H}_{2} \mathrm{PO}_{4}\right]^{0}$ and $\left[\mathrm{D}_{2} \mathrm{PO}_{4}\right]^{0}$ centers). In contrast, there are no reports in the literature for KDP or DKDP that describe a radiation-induced band peaking at $230 \mathrm{~nm}$. We note that the 230-nm band in Figure 6.1 is not the impurity-related band at $215 \mathrm{~nm}$ described earlier in KDP [68].

In Figure 6.3, I present a temperature dependence of the intensities of the bands at 230,390 , and $550 \mathrm{~nm}$. In an effort to present a temperature dependence of the intensities of the optical absorption bands we realized this is not an easy task since these are broad bands and they are close to each other in peak position, thus their contribution overlap. This is 


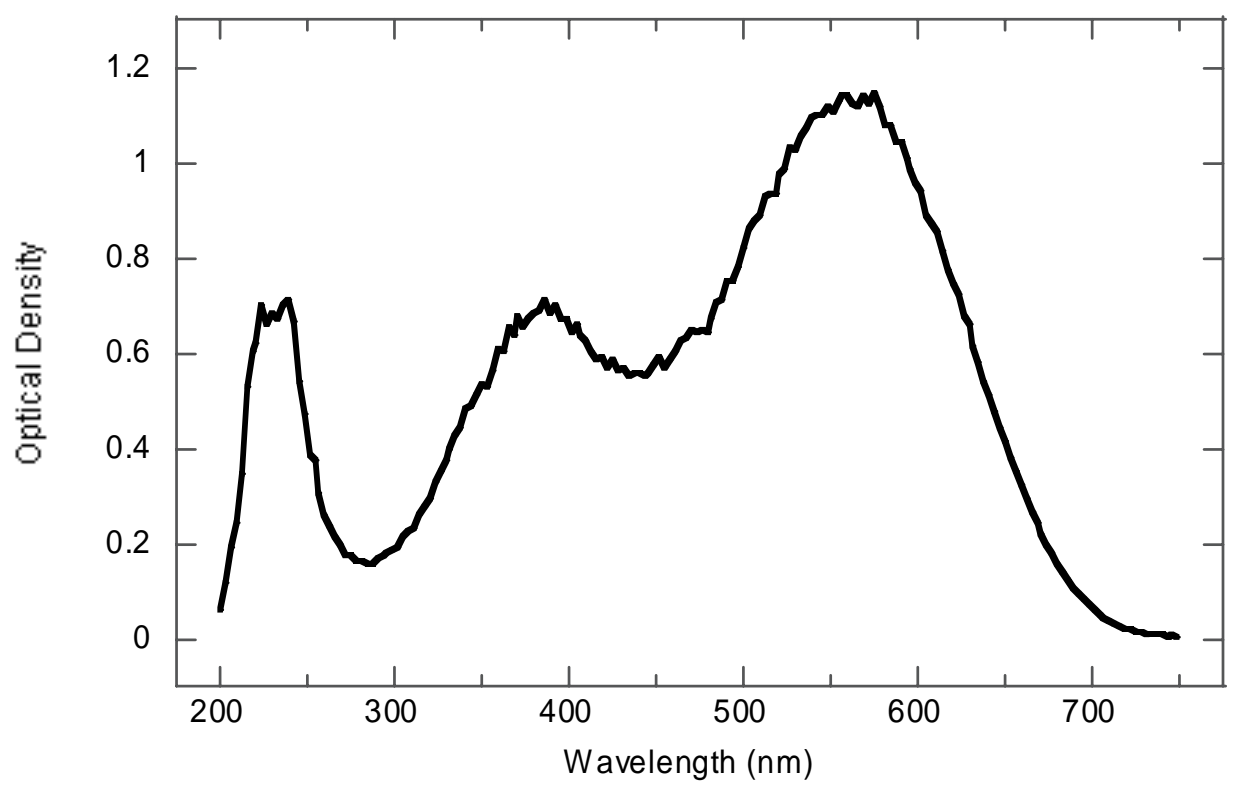

Figure 6.2. Absorption spectrum showing only the representative optical bands at 230, 390, and $550 \mathrm{~nm}$ formed after $\mathrm{x}$ rays irradiation and before other absorption bands are formed. This spectrum was obtained by subtracting the spectrum at $120 \mathrm{~K}$ from the spectrum taken at $80 \mathrm{~K}$.

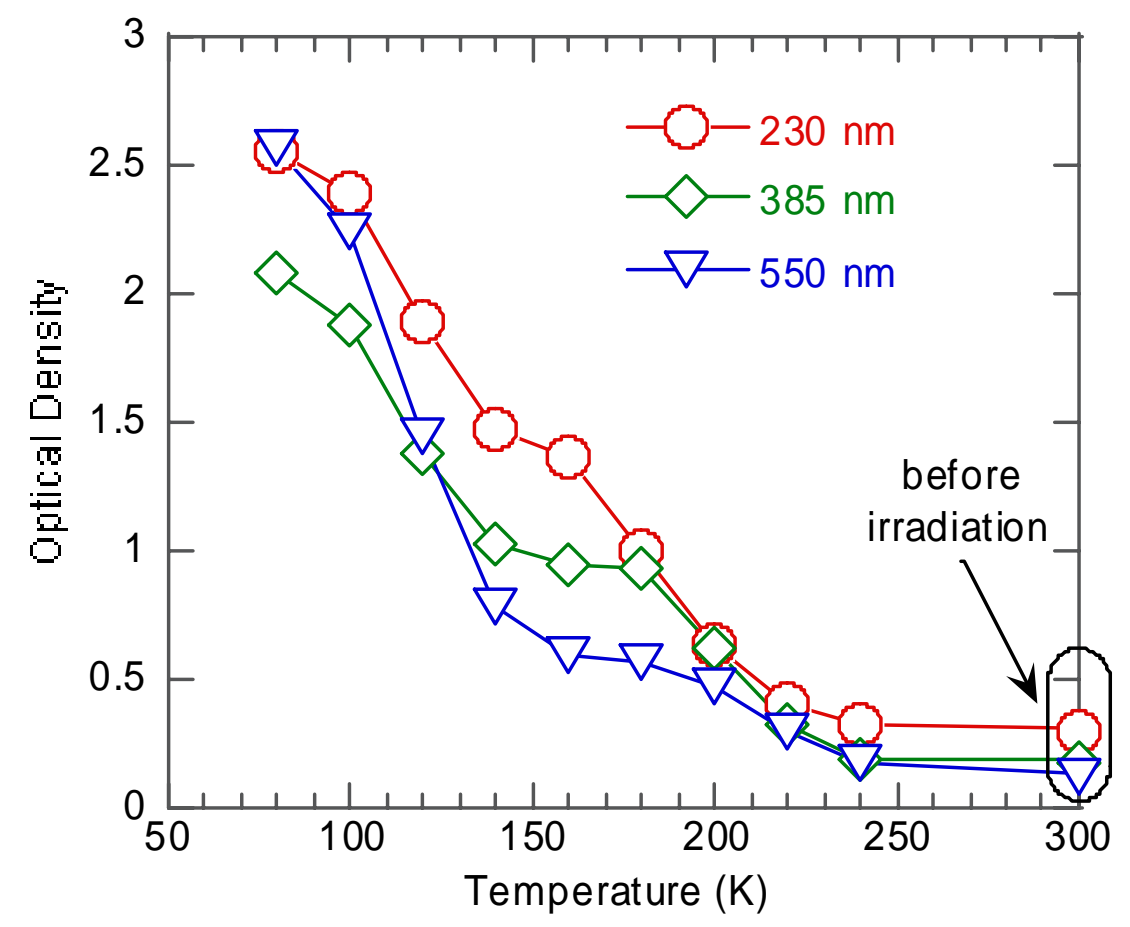

Figure 6.3. Temperature dependence of the intensity of absorption bands formed in DKDP crystal at 230, 390, and $550 \mathrm{~nm}$ after the $77 \mathrm{~K}$ irradiation. 
specially valid for the band peaking at $450 \mathrm{~nm}$. Overall the intensity of this band decreases with each annealing step; however the rate of decreasing is different for the 450-nm band than it is for the other three bands. This behavior can be observed in Figure 6.1. After the $200 \mathrm{~K}$ annealing step, which is approximately the Curie temperature for DKDP crystals, the base line for optical absorption spectra changes and it becomes more difficult to precisely measure the intensity at a certain wavelength. The general trend is however easy to follow because there is an abrupt decrease in all the absorption bands and the overall intensity decreases.

The irradiation at $77 \mathrm{~K}$ with $\mathrm{x}$ rays produced four dominant EPR spectra in the DKDP crystal. Figure 6.4 shows the self-trapped hole, $\left[\mathrm{D}_{2} \mathrm{PO}_{4}\right]^{0}$, and the hole trapped adjacent to a deuterium vacancy, $\left[\mathrm{DPO}_{4}\right]^{-}$. These data were taken at $40 \mathrm{~K}$ with the magnetic field parallel

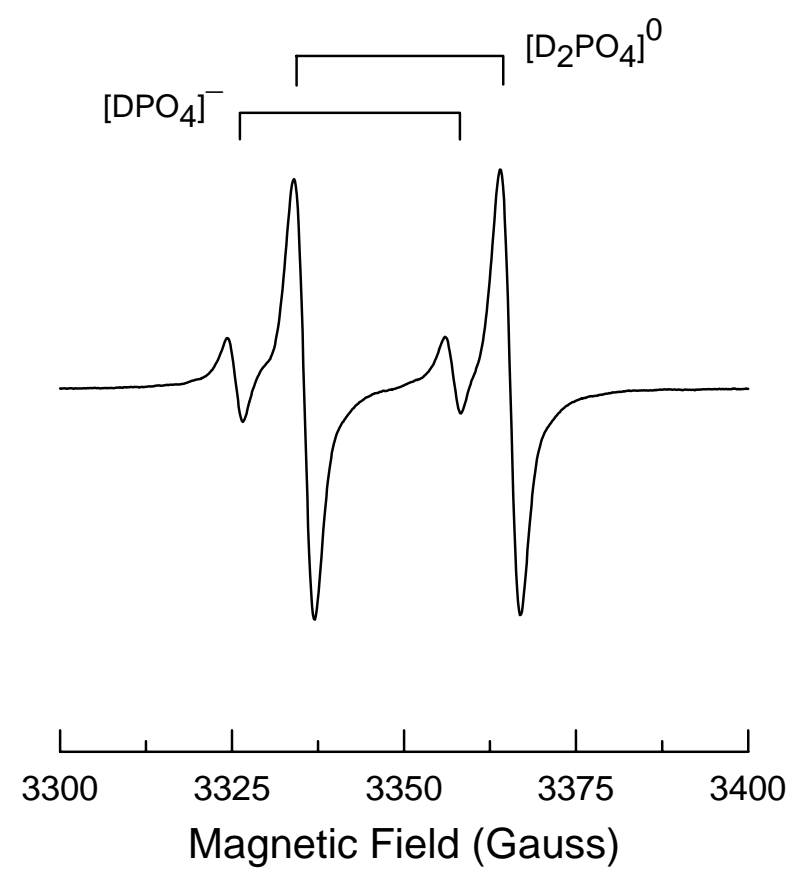

Figure 6.4. EPR spectrum showing the $\left[\mathrm{D}_{2} \mathrm{PO}_{4}\right]^{0}$ and $\left[\mathrm{DPO}_{4}\right]^{-}$centers in a DKDP crystal after an irradiation at $77 \mathrm{~K}$ with $\mathrm{x}$ rays. These data were taken at $40 \mathrm{~K}$ with the magnetic field parallel to the $\mathrm{c}$ axis. 
to the $\mathrm{c}$ axis of the crystal. Both of these centers have one unpaired spin $(\mathrm{S}=1 / 2)$, and they both show a large hyperfine interaction with one phosphorus nucleus $\left({ }^{31} \mathrm{P}\right.$ has $\mathrm{I}=1 / 2$ and is $100 \%$ abundant). The distinguishing feature of the two spectra is the $g$ value; the $\left[\mathrm{D}_{2} \mathrm{PO}_{4}\right]^{0}$ center has an effective $g_{c}$ value of 2.0164 and the $\left[\mathrm{DPO}_{4}\right]^{-}$center has an effective $\mathrm{g}_{\mathrm{c}}$ value of 2.0217. As previously reported by Stevens et al. [62], the self-trapped hole center in DKDP has the unpaired spin shared equally by two adjacent oxygen ions within a $\mathrm{PO}_{4}$ unit. In contrast, the hole trapped by a deuterium vacancy has the unpaired spin localized on only one oxygen ion (in particular, the oxygen ion nearest the vacancy).

Figure 6.5 shows the EPR spectra from the deuterium atoms $\left(\mathrm{D}^{0}\right)$ and hydrogen atoms $\left(\mathrm{H}^{0}\right)$ that were produced in our DKDP crystal by the irradiation at $77 \mathrm{~K}$ with $\mathrm{x}$ rays. These data were taken at $40 \mathrm{~K}$ with the magnetic field parallel to the $\mathrm{c}$ axis of the crystal. The set of

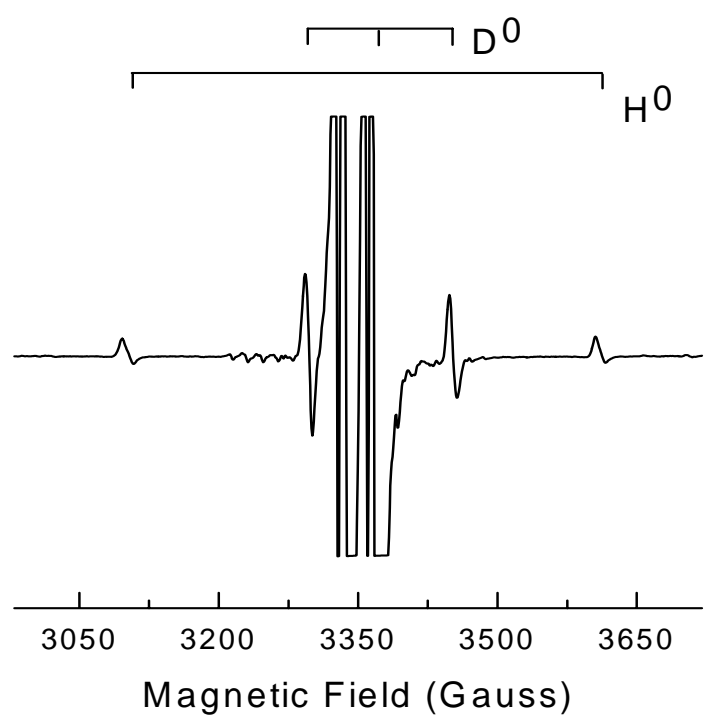

Figure 6.5. EPR spectrum showing deuterium atoms $\left(\mathrm{D}^{0}\right)$ and hydrogen atoms $\left(\mathrm{H}^{0}\right)$ in a DKDP crystal after an irradiation at $77 \mathrm{~K}$ with $\mathrm{x}$ rays. These data were taken at $40 \mathrm{~K}$ with low microwave power. 
intense lines that go off scale in the middle of Figure 6.5 are from the two hole centers shown in Figure 6.4. To reduce the effects of saturation on the EPR signals from the $\mathrm{D}^{0}$ and $\mathrm{H}^{0}$ atoms, the microwave power used in Figure 6.5 was approximately twelve times smaller than the microwave power used in Figure 6.4. Even with the lower power, long spin-lattice relaxation times cause an easily observed distortion of the EPR line shapes in the $\mathrm{D}^{0}$ and $\mathrm{H}^{0}$ spectra. The $\mathrm{H}^{0}$ spectrum contains two lines because of the $I=1 / 2$ nuclear spin of the proton (i.e., the number of lines is $2 I+1$ ), while the $\mathrm{D}^{0}$ spectrum contains three lines because of the $I=1$ spin of the deuteron. One of the three $\mathrm{D}^{0}$ lines in Figure 6.5 is "buried" under the set of hole-center lines in the middle of the trace. Although our crystal was deuterated during growth, there is a small amount of hydrogen present as verified by the $\mathrm{H}^{0}$ spectrum in Figure 6.5. It is important to note that the relative intensities of the $\mathrm{D}^{0}$ and $\mathrm{H}^{0}$ centers in Figure 6.5 probably do not provide information about the degree of deuteration of the crystal. We say this because the formation of $\mathrm{H}^{0}$ centers during an irradiation is expected to be a more likely event than the formation of $\mathrm{D}^{0}$ centers (this is based on the difference in mass and thus the rate at which the hydrogen and deuterium atoms will escape, i.e., diffuse, from their regular lattice sites) [61].

The $\mathrm{D}^{0}$ and $\mathrm{H}^{0}$ centers are the electron traps that complement the previously described $\left[\mathrm{D}_{2} \mathrm{PO}_{4}\right]^{0}$ and $\left[\mathrm{DPO}_{4}\right]^{-}$hole traps. Since the crystal remains electrically neutral after an irradiation, the total number of hole centers must equal the total number of electron centers. We found this to be true in our DKDP crystal. Warming the crystal to higher temperatures provided information about the relative stabilities of these electron and hole traps, as they either recombined with each other or converted to new defects. To obtain the most useful results, we monitored the EPR signals during a series of isochronal pulse anneals. At each step, the signals were recorded at $40 \mathrm{~K}$. These results are shown in Figure 6.6. After the initial irradiation at $77 \mathrm{~K}$ with $\mathrm{x}$ rays, the EPR spectra were measured at $40 \mathrm{~K}$ (this provides the data points at $77 \mathrm{~K}$ in Figure 6.6). Then the crystal was warmed to $80 \mathrm{~K}$, held 


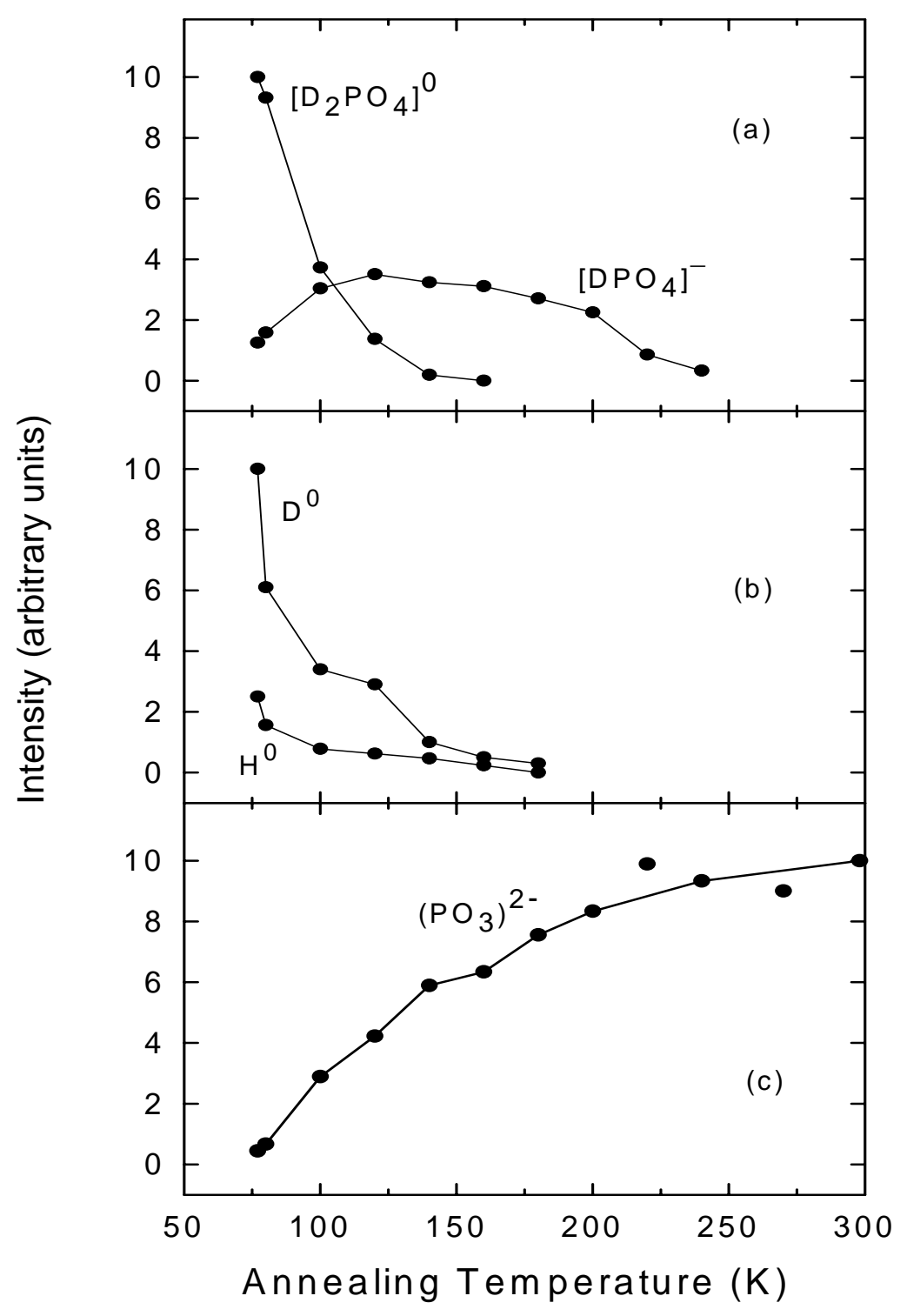

Figure 6.5. Thermal anneal of the EPR spectra produced in DKDP by an irradiation with $\mathrm{x}$ rays at $77 \mathrm{~K}$. The spectra were monitored at $40 \mathrm{~K}$ after being sequentially held at each higher temperature for ten min. (a) The $\left[\mathrm{D}_{2} \mathrm{PO}_{4}\right]^{0}$ and $\left[\mathrm{DPO}_{4}\right]^{-}$hole centers. (b) The deuterium $\left(\mathrm{D}^{0}\right)$ and hydrogen $\left(\mathrm{H}^{0}\right)$ atoms. (c) $\mathrm{A}\left(\mathrm{PO}_{3}\right)^{2-}$ oxygen-vacancy center. 
there for ten min, and returned to $40 \mathrm{~K}$ for another measurement. This process was repeated for anneal temperatures of $100 \mathrm{~K}, 120 \mathrm{~K}, 140 \mathrm{~K}$, etc. until the sample had been taken to room temperature. Our goal was to have the EPR anneal sequence be comparable to the sequence of optical anneals described in Figure 6.1.

The $\left[\mathrm{D}_{2} \mathrm{PO}_{4}\right]^{0}$ self-trapped hole centers and the $\mathrm{D}^{0}$ deuterium atoms are the dominant point defects present immediately after the irradiation at $77 \mathrm{~K}$, as illustrated in Figures 6.6(a) and 6.6(b). These hole and electron traps quickly decay as the temperature is increased. After the $100-\mathrm{K}$ anneal, about $35 \%$ of the deuterium atoms and nearly $40 \%$ of the selftrapped holes remain. The thermal decay of the hydrogen atoms occurs in the same range as the decay of the deuterium atoms. These three defects are almost completely gone after the 160-K anneal. As the self-trapped hole centers decay, the concentration of vacancyassociated $\left[\mathrm{DPO}_{4}\right]^{-}$hole centers increases, thus indicating a migration of a portion of the holes to the more stable trapping site. These latter centers then decay in the 160 to $240 \mathrm{~K}$ range, as shown in Figure 6.6(a).

An EPR signal from oxygen vacancies [66] was also observed in our DKDP crystal. It is very small after the initial irradiation at $77 \mathrm{~K}$, but then grows significantly in intensity as the crystal is annealed to higher temperatures. The paramagnetic oxygen vacancies are referred to as $\left(\mathrm{PO}_{3}\right)^{2-}$ centers in KDP, and we use the same notation in DKDP. Although five distinct $\left(\mathrm{PO}_{3}\right)^{2-}$ centers were observed in an earlier study in KDP [66], only one center of this type dominates in our crystal during the sequence of anneals from $77 \mathrm{~K}$ to room temperature. Figure 6.7 shows the doublet EPR spectrum from this $\left(\mathrm{PO}_{3}\right)^{2-}$ center following the thermal anneal at $200 \mathrm{~K}$. These data were taken at $40 \mathrm{~K}$ with the magnetic field parallel to the $\mathrm{c}$ axis of the crystal. The spectrum has a ${ }^{31} \mathrm{P}$ hyperfine splitting of approximately $744 \mathrm{G}$. The complete set of data showing the growth of this $\left(\mathrm{PO}_{3}\right)^{2-}$ center as a function of the anneal temperature is provided in Figure 6.6(c). In addition to the doublet from the $\left(\mathrm{PO}_{3}\right)^{2-}$ center, there are EPR lines in the middle of the spectrum in Figure 6.7 from the $\left[\mathrm{DPO}_{4}\right]^{-}$hole 
center and from very low concentrations of unidentified perturbed hole centers [61] similar in structure to the $\left[\mathrm{DPO}_{4}\right]^{-}$hole center.

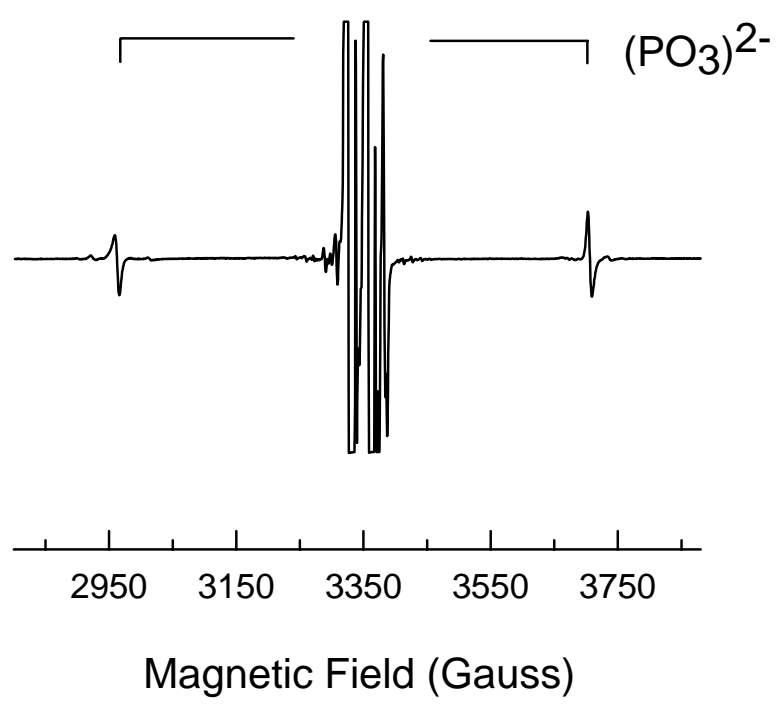

Figure 6.7. EPR spectrum showing an oxygen-vacancy $\left(\mathrm{PO}_{3}\right)^{2-}$ center in a DKDP crystal that had first been irradiated at $77 \mathrm{~K}$ with $\mathrm{x}$ rays and then held at $200 \mathrm{~K}$ for ten min. These data were taken at $40 \mathrm{~K}$ with the magnetic field parallel to the $\mathrm{c}$ axis.

As a final step to complete our thermal anneal sequence, we held the DKDP sample at room temperature for approximately $18 \mathrm{~h}$ (i.e., overnight) and then took a final EPR spectrum at room temperature. This spectrum is shown in Figure 6.8. It contains two $\left(\mathrm{PO}_{3}\right)^{2-}$ centers, with phosphorus hyperfine splittings of 736 and $648 \mathrm{G}$. They are very similar to be the $\left(\mathrm{PO}_{3}\right)_{\mathrm{B}}{ }^{2-}$ and $\left(\mathrm{PO}_{3}\right)_{\mathrm{D}}{ }^{2-}$ centers previously described by Garces et al. [66], and we use this latter notation in Figure 6.8. In addition to the oxygen-vacancy electron traps, there is also a holelike center in Figure 6.8. The pair of lines, split by approximately $7.1 \mathrm{G}$, 
in the middle of this spectrum are from the silicon-associated hole center [66], i.e., a hole trapped on an oxygen ion adjacent to a silicon substituting for a phosphorus ion. We suggest that these new hole centers are formed when the $\left[\mathrm{DPO}_{4}\right]^{-}$centers become thermally unstable above $200 \mathrm{~K}$, thus allowing a portion of the holes to migrate to the more stable silicon trapping sites. The silicon-hole center was first observed in KDP crystals and the 7.1-G hyperfine splitting was attributed to an adjacent hydrogen [66]. This raises a significant

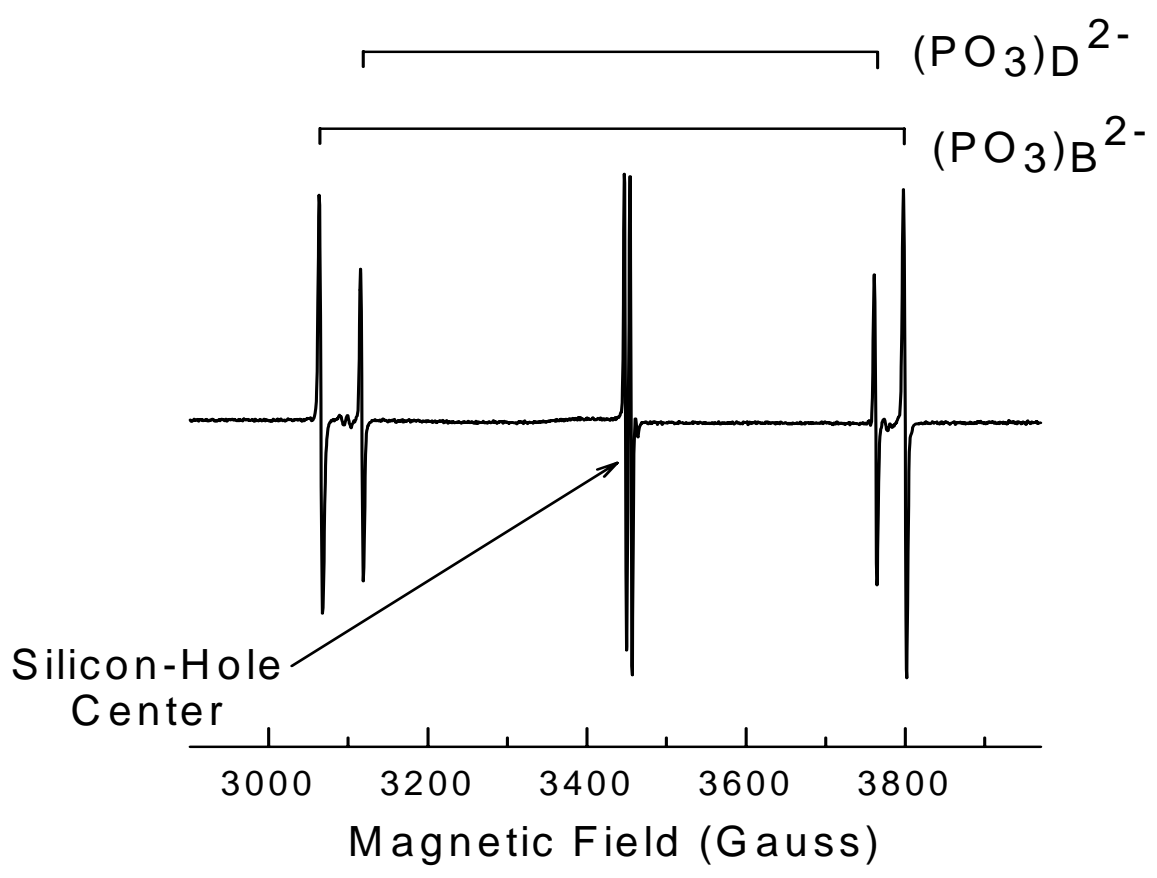

Figure 6.7. EPR spectrum showing the $\left(\mathrm{PO}_{3}\right)_{\mathrm{B}}{ }^{2-}$ and $\left(\mathrm{PO}_{3}\right)_{\mathrm{D}}{ }^{2-}$ electron centers and the silicon-associated hole center in a DKDP crystal that had first been irradiated at $77 \mathrm{~K}$ with $\mathrm{x}$ rays and then held for $18 \mathrm{~h}$ at room temperature. These data were taken at room temperature with the magnetic field parallel to the $\mathrm{c}$ axis. 
question. In Figure 6.8, only the hydrogen version of the silicon-hole center is present. We had expected to see a large EPR signal representing the deuterium-version of this defect (with the same g value but a much reduced and perhaps unresolved hyperfine splitting due to the smaller magnetic moment of the deuterium nucleus) after our DKDP crystal was warmed to room temperature. A possible, but not completely satisfying, explanation of the trapped hole data in Figure 6.8 is based on the relative thermal stabilities of the deuterium and hydrogen versions of the silicon-hole centers. In this scenario, both versions of the center would form in the 200 to $240 \mathrm{~K}$ temperature range as holes migrate to the silicon, but only the hydrogen version would be stable, and thus survive, at room temperature. Because of interference from the EPR signals from the residual amounts of the $\left[\mathrm{DPO}_{4}\right]^{-}$hole center and the other similar weak perturbed hole centers (see the previous paragraph) as the crystal was annealed at 220,240 , and $260 \mathrm{~K}$, we were unable to establish whether this proposed explanation was correct.

\subsection{Discussion and conclusions}

The production and thermal decay of optically active point defects in DKDP crystals are subjects of considerable interest because of the various applications of this material that involve high power laser beams in the visible and ultraviolet. Since these are normally room temperature applications, the majority of point defects formed by the laser beams participate in only a transient manner $[55,56]$. Our present efforts have focused on understanding the defects that contribute to this room-temperature transient optical response of DKDP. Specifically, we formed point defects at $77 \mathrm{~K}$ and then monitored their behavior as the crystal was systematically warmed to room temperature. The defects in our study were produced with $\mathrm{x}$ rays (it is known that the 266-nm fourth harmonic of a Nd:YAG laser will produce these same defects, although with less efficiency than the $\mathrm{x}$ rays) $[61,62,66]$. A unique 
feature of our work is the close correlation of optical absorption and EPR data.

Our results suggest that the following processes occur in DKDP crystals. When this material is exposed to ionizing radiation at $77 \mathrm{~K}$, large numbers of "free" electrons and holes are formed. These holes will immediately become localized within the lattice by forming the $\left[\mathrm{D}_{2} \mathrm{PO}_{4}\right]^{0}$ self-trapped hole centers (i.e., the hole is shared by two adjacent oxygen ions with no defects nearby). The EPR spectrum from the $\left[\mathrm{D}_{2} \mathrm{PO}_{4}\right]^{0}$ centers in Figure 6.4 verifies that these are the dominant hole centers created by the $77-\mathrm{K}$ irradiation. This also allows us to assign the two optical absorption bands, at 390 and $550 \mathrm{~nm}$, to the $\left[\mathrm{D}_{2} \mathrm{PO}_{4}\right]^{0}$ centers because these peaks dominate the visible and near-ultraviolet regions of the absorption spectrum taken immediately after the 77-K irradiation (see trace $\mathrm{b}$ in Figure 6.1). This assignment was previously suggested by Dieguez et al. [63,64], and we support their conclusions. Our present data provides additional and more convincing evidence because the EPR and optical absorption measurements were made on adjacent samples that had been cut from one boule and then subjected to nearly identical irradiation conditions. The significant similarities in the thermal decay of the $\left[\mathrm{D}_{2} \mathrm{PO}_{4}\right]^{0}$ EPR signal, shown in Figure 6.6(a), and the thermal decay of the optical absorption, shown in Figure 6.1, further support the assignment of the 390 and $550 \mathrm{~nm}$ bands to the $\left[\mathrm{D}_{2} \mathrm{PO}_{4}\right]^{0}$ self-trapped hole centers. Both the EPR signal and the two absorption bands rapidly decay in the 80 to $120 \mathrm{~K}$ range.

Many of the "free" electrons produced by the irradiation at $77 \mathrm{~K}$ will immediately recombine with self-trapped hole centers. However, a portion of these electrons will join with deuterium ions located at their normal sites between pairs of oxygen ions and form deuterium atoms. Once a deuterium atom forms at the regular site, it will then migrate away to an interstitial position. Since our crystals were not $100 \%$ deuterated, a few hydrogen atoms are also formed in this same fashion. The EPR spectra in Figure 6.5 verify that deuterium $\left(\mathrm{D}^{0}\right)$ and hydrogen $\left(\mathrm{H}^{0}\right)$ atoms were produced by the initial $77-\mathrm{K}$ irradiation. This then allows us to assign the optical absorption band at $230 \mathrm{~nm}$ to deuterium (and hydrogen) atoms because this 
band is the remaining dominant peak in the absorption spectrum taken immediately after the 77-K irradiation (trace $\mathrm{b}$ in Figure 6.1). We suggest that this absorption band represents the localized transfer of an electron from a neighboring substitutional oxygen in to the interstitial deuterium atom. In support of this assignment, we note that hydrogen atoms in other wideband-gap materials have been associated with optical absorption bands in the 200 to $250 \mathrm{~nm}$ region [73-76]. The thermal anneal results in Figure 6.1, Figure 6.3, and Figure 6.6(b) also support this assignment by showing that the $\mathrm{D}^{0}$ and $\mathrm{H}^{0}$ atoms and the $230-\mathrm{nm}$ band decay in a similar fashion in the 80 to $140 \mathrm{~K}$ range.

A series of thermal anneals from $80 \mathrm{~K}$ to room temperature allowed us to monitor the decay of the initial electron and hole traps and to observe the migration of a portion of these charges to new trapping sites. The electrons and holes that were trapped in the form of $\mathrm{D}^{0}$ and $\mathrm{H}^{0}$ atoms and $\left[\mathrm{D}_{2} \mathrm{PO}_{4}\right]^{0}$ self-trapped hole centers quickly become thermally unstable when the crystal is heated above $77 \mathrm{~K}$. At this stage, a large number of deuterium (and proton) vacancies are present in the crystal, either formed during growth as charge compensators for oxygen vacancies or formed by the ionizing radiation when the deuterium atoms and hydrogen atoms move into interstitial positions. As shown in Figure 6.6(a), these vacancies will then trap many of the holes being released by the $\left[\mathrm{D}_{2} \mathrm{PO}_{4}\right]^{0}$ centers and form $\left[\mathrm{DPO}_{4}\right]^{-}$centers. The increase in the number of $\left[\mathrm{DPO}_{4}\right]^{-}$centers coincides with the appearance of the broad optical absorption band peaking near $450 \mathrm{~nm}$ (trace e in Figure 6.1). Also, the $\left[\mathrm{DPO}_{4}\right]^{-}$EPR signal and the absorption band at $450 \mathrm{~nm}$ have nearly identical thermal characteristics, i.e., both have disappeared when the crystal reaches $260 \mathrm{~K}$. For these reasons, we assign the 450-nm absorption band to the vacancy-associated [ $\left.\mathrm{DPO}_{4}\right]^{-}$centers.

As the $\left[\mathrm{DPO}_{4}\right]^{-}$centers decay in the 200 to $240 \mathrm{~K}$ region, a portion of these released holes migrates to the silicon trapping sites. The resulting silicon-hole centers are then stable to near room temperature (as discussed earlier, the hydrogen version may be slightly more stable than the deuterium version). Electron traps also evolve as the crystal is warmed. The 
$\mathrm{D}^{0}$ and $\mathrm{H}^{0}$ atoms become thermally unstable, and a portion of the released electrons migrates to the small number of oxygen vacancies already present in the crystal from growth. This results in $\left(\mathrm{PO}_{3}\right)^{2-}$ centers that are stable for many hours at room temperature. As described in Section 6.1, these silicon-associated hole centers and $\left(\mathrm{PO}_{3}\right)^{2-}$ oxygen vacancy electron centers are most likely responsible for the relatively small amount of radiation-induced optical absorption that remains in the 400 to $200 \mathrm{~nm}$, after warming to room temperature.

In summary, we have shown in a relative sense that the $\mathrm{D}^{0}$ and $\mathrm{H}^{0}$ atoms and $\left[\mathrm{D}_{2} \mathrm{PO}_{4}\right]^{0}$ self-trapped hole centers have very short lifetimes at room temperature, the $\left[\mathrm{DPO}_{4}\right]^{-}$vacancy-associated hole centers have intermediate lifetimes, and the $\left(\mathrm{PO}_{3}\right)^{2-}$ oxygen-vacancy centers and silicon-hole centers have long lifetimes. Although we cannot provide numerical values for these room-temperature lifetimes, our results provide a better understanding of the contribution each point defect may make to the transient visible and ultraviolet absorption produced in DKDP by high-power pulsed lasers. We especially suggest that the nonlinear optical performance of DKDP crystals may be improved by minimizing the number of oxygen vacancies and silicon impurities incorporated during growth. Their longer lifetimes at room temperature will allow absorption below $400 \mathrm{~nm}$ to steadily accumulate over a series of pulses and ultimately lead to device failure. 


\section{CHAPTER 7}

\section{COMPUTATIONAL MODELING OF POINT DEFECTS IN $\mathrm{KH}_{2} \mathrm{PO}_{4}$}

This chapter contains results obtained from modeling several of the important point defects in $\mathrm{KH}_{2} \mathrm{PO}_{4}$ crystals. The quantum-mechanical calculations were performed using Gaussian 98 for Microsoft Windows and graphical outputs from these computations were provided by GaussView. Descriptions of the theoretical approach and the methodology of calculation are given in the first part of the chapter and the results of the calculations are given in the second part of the chapter. The purpose of this work is to show the correlations between the experimental hyperfine data from electron paramagnetic resonance measurements and the predictions emerging from the calculations. The good agreement between the experiments and the calculations provides another way to verify the interpretations of the experimental data, and it gives confidence that the defect models we suggest are correct.

\subsection{Technical approach}

Gaussian 98 [70] is a commercially available computational chemistry software package which uses electronic structure methods to perform basic types of calculations such as computing the total potential energy of a particular molecular structure (spatial arrangement of nuclei and electrons) and performing geometry optimizations (locating the lowest energy molecular structure in close proximity to the specified starting structure). The program uses the principles of quantum mechanics as the basis for the computations. There are two major classes of electronic structure methods used in Gaussian calculations: the semi-empirical methods and ab initio methods. Semi-empirical methods use parameters 
derived from experimental data in order to obtain a simple approximation to the Schrödinger equation. The term ab initio ("from the beginning") is used to describe calculations in which the following three steps are all explicitly performed: 1) write down the Hamiltonian operator for the system; 2) select some mathematical functional form as the trial wavefunction (this form should have variable parameters); 3) minimize the energy with respect to variations in the parameters [71]. Ab initio molecular orbital theory is involved in predicting properties of atomic and molecular systems such as energy of ground or excited states, atomic orbitals, atomic charges, etc. Ab initio calculations are based upon the fundamental laws of quantum mechanics and use a variety of mathematical transformation and approximation techniques to solve the fundamental equations. Based on these methods, the Gaussian program includes many different theoretical models, also called model chemistries. Model chemistries are characterized by the combination of a theoretical procedure and a set of basis functions. Every calculation performed with Gaussian must specify the desired theoretical model chemistry in addition to specifying the molecular system to be considered and which results to be computed. One of the most commonly used theoretical methods is the Hartree-Fock (HF) Self-Consistent Field. Another method is Møller-Plesset (MP) perturbation theory which, in contrast with Hartree-Fock theory, takes into account the electron correlation, is a non-iterative method, and adds higher order corrections to energy (through fifth-order).

The Hartree-Fock approach takes advantage of the variational principle, which says that for the ground state of any antisymmetric normalized function of the electronic coordinates, the expectation value for the energy will always be greater than the energy for the exact wavefunction. Thus, the problem becomes one of finding the set of coefficients that minimize the energy of the resultant wavefunction. The Self-Consistent Field (SCF) method is used to compute equations that are not linear and must be solved iteratively. At convergence, the energy is at a minimum, and the orbitals generate a field which produces the same type of orbitals, accounting for the method's name. During the calculation with this 
method a test of convergence takes place. If it fails, the strategy is to begin a new iteration. If it succeeds, it goes on to perform other parts of the calculation like population analysis, for example.

Along with the theoretical method a basis set needs to be selected in the input of the calculation. A basis set is used to mathematically represent the molecular orbitals within a molecule. Larger basis sets impose fewer constraints on electrons and more accurately approximate the exact molecular orbitals. The molecular orbitals are expressed as linear combinations of the pre-defined set of one-electron functions known as basis functions. These basis functions are usually centered on the atomic nuclei and so bear some resemblance to atomic orbitals. Gaussian, like other ab initio electronic structure programs, uses gaussian-type atomic functions as basis functions. Gaussian functions have the general form $g(\alpha, \vec{r})=c x^{n} y^{m} z^{l} e^{-\alpha r^{2}}$ where $c$ is the normalization constant, $\alpha$ is a constant determining the size (radial extent) of the function, and $n, m$, and $l$ are constants which can make the gaussian function resemble the atomic orbitals (for example, an s-type of orbital if $n=m=l$ $=0$, a p pype if $n=l=0$ and $m=1$, or $\mathrm{d}_{\mathrm{xy}}$ - type of orbital if $n=0$ and $m=l=1$ ). Linear combinations of primitive gaussian are used to form contracted gaussians which then are used to construct the basis set of functions and then the molecular orbitals.

There is a third component to every Gaussian calculation involving how electron spin is handled: whether the calculation is performed using an open shell (unequal numbers of spin up and spin down electrons) model or a closed shell (even number of electrons divided into pairs of opposite spin) model. The two options are also referred to as unrestricted and restricted calculations, respectively. Restricted closed-shell calculations force each electron pair into a single spatial orbital, while open-shell calculations use separate spatial orbitals for the spin up and spin down electrons (alpha and beta respectively).

The Gaussian calculations that I have performed for this study are single point energy calculations and geometry optimizations. A single point energy calculation is a prediction of 
the energy and related properties for a molecule with a specified geometry structure. The energy is defined as the sum of the electronic energy and nuclear repulsion energy of the molecule at a specified nuclear configuration. This is often called the total energy. The phrase single point is the key, since this calculation is performed at a single, fixed point on the potential energy surface for the molecule. The purposes of performing single point energy calculations include the following: to obtain basic information about a molecule; as a consistency check on a molecular geometry to be used as the starting point for an optimization; to compute very accurate values for the energy and other properties for a molecular unit that had been previously geometry optimized at a lower level of theory.

To run a Gaussian calculation the following steps are necessary: load or enter the Gaussian input, start execution of the job, and finally examine and interpret the output. The Gaussian input has the schematic structure described in Table 7.1.

Table 7.1. The basic structure of a Gaussian 98 input file.

\begin{tabular}{ll}
\hline Input Section & Contents \\
\hline Route Section & Specifies the job type and model chemistry. \\
\hline Title Section & Describes the job for the output. \\
\hline Charge and Multiplicity & Charge and spin multiplicity must be specified. \\
\hline Molecule Specification & Gives the structure of the molecule to be studied. \\
\hline Variables Section & Specifies values for the variables used in the \\
& molecule specification. \\
\hline
\end{tabular}

The route section of a Gaussian input file specifies the kind of job to be run as well as the specific theoretical method and basis set which should be used and the desired type of calculation. All of these items are specified via keywords. An example of a route section is:

The Route Section UHF/6-31G Pop=Full Opt=(Z-Matrix) NoSymm 
The explanation for each keyword, in this case, is as follows: UHF stands for unrestricted Hartree-Fock theoretical level which means an open shell calculation using basis set 6-31G. This basis set applies to atoms from $\mathrm{H}$ to $\mathrm{Kr}$. The 6-31G basis set has three shells. A shell is a set of basis functions. Gaussian 98 supports shells of arbitrary angular momentum: s, p, d, f, and so on. One shell in this basis set is a linear combination of six primitive s-type gaussians. The second shell is a combination of three primitive sp-shells. The third shell consists of a single sp-function. This basis set also supports polarization functions and diffuse functions. Polarization functions are essentially added orbital functions with angular momentum beyond what is required for the ground state of each atom (for example, $\mathrm{d}$ functions for carbon atom, or $\mathrm{p}$ functions for hydrogen atom). These functions are common for calculations extending up to medium-sized systems. Diffuse functions are large-size versions of s- or p- type functions which allow orbitals to occupy a larger region of space. Diffuse functions are important for systems with anions and other systems with significant negative charge, or systems in excited states. The next keyword, Pop=Full, represents the specification for displaying all molecular orbital coefficients. Opt $=(Z-M a t r i x)$ is the keyword for a geometry optimization calculation with the specification that the coordinates of the atoms forming the molecule under consideration should remain in the same format as the one introduced in the initial molecule specification. For example, I have used Cartesian coordinates in the molecule specification section to indicate the positions of each atom in the cluster, and in the output the result for the new positions also will be in Cartesian coordinates. The next keyword, NoSymm, imposes a restriction on the calculation that during the running of the input the Gaussian program should not reorient the symmetry axes along specific directions and should not move the origin of the system to the center of charge. No special keyword for single point energy calculation is needed in the route section since this is the default calculation in Gaussian. Simply specifying a procedure and basis set in the route section requests a single point calculation. 
After the route section, the title section is next. This section of the input file consists of one or more lines describing the calculation in any way the user desires. As an example, here is a title that I have used for a single point energy calculation for a cluster of atoms in $\mathrm{KH}_{2} \mathrm{PO}_{4}$ in the tetragonal crystalline structure.

The Title Section KDP Energy Calculation / Cluster Structure Tetragonal

The charge and spin multiplicity is the next information needed to be specified in the input file. The charge is a positive or negative integer specifying the total charge on the molecule. Thus, 1 or +1 would be used for a cluster with one unit of excess positive charge, - 1 designates a cluster with one unit of excess negative charge, and 0 represents a neutral cluster. The spin multiplicity for a molecule is given by the equation $2 S+1$, where $\mathrm{S}$ is the total spin for the molecule. Thus, a singlet (a system with no unpaired electrons) has a spin multiplicity of 1 , a doublet (one unpaired electron) has a spin multiplicity of 2 , a triplet (two unpaired electrons of like spin) has a spin multiplicity of 3 , and so on.

The structure of the molecular system to be investigated appears in the molecule specification section, after the charge and spin multiplicity line. This is the section where the coordinates of each atom from the molecule or cluster must be specified using either (a) Cartesian coordinates, (b) Z-matrix (internal coordinates which specifies the locations of atoms and bonds between atoms using bond lengths, bond angles, and dihedral angles), or (c) mixed internal and Cartesian coordinates. In this study, Cartesian coordinates were used and the input consists of a series of lines containing the atomic symbol of an atom and its $\mathrm{x}, \mathrm{y}$, and $\mathrm{z}$ coordinates. Another way of writing the coordinates in the molecule specification section is to use variables. This is especially useful when a geometry optimization calculation is needed. Variable names are substituted for numerical values within the list of Cartesian coordinates and their values are then defined in a separate section following the coordinates. The two sections are separated by a blank line and the label Variables. When 
two or more atoms have the same coordinates in absolute value but opposite in sign, due to the symmetry of the molecule, the variables should use the same symbol with the sign "_." in front. To illustrate this case, I am giving one example from the calculation performed for KDP. Due to the symmetry of the crystal, there are four oxygen ions surrounding the phosphorus ion located at the corners of a nearly regular tetrahedron. For one $\mathrm{PO}_{4}$ group only, the Cartesian coordinates can be written as follows:

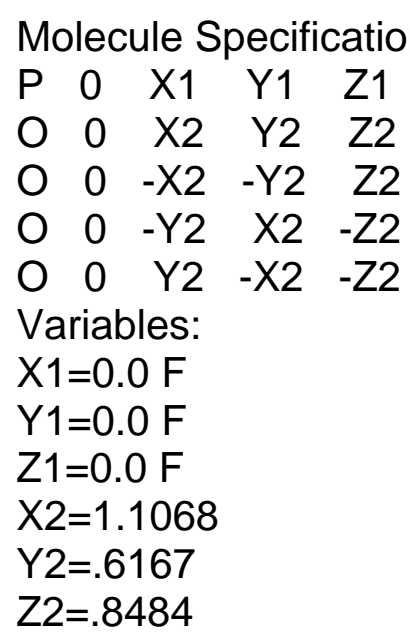

The symbol "F" after the variable means a frozen variable, thus during the calculation this variable will not change its value. If the symbol " $F$ " is not indicated on a variable it means that the respective variable is allowed to change during a geometry optimization. By default, the units for lengths are angstroms and for angles are degrees. Energies are measured in hartrees, defined as the Coulomb repulsion between two electrons separated by 1 bohr (1bohr $\cong 0.5292 \AA$ ). Masses are also specified in electron mass unit (i.e., define $\mathrm{m}_{\mathrm{e}}=1$ ).

The Gaussian output file of a calculation is available only after a Gaussian job has been completed. Some of the main features of a Gaussian output file will be described here. The beginning of the file contains the route section, title section, and molecule specification as they appear in the input file. Next, the standard orientation is given, which is the coordinate system used internally by the program as it performs the calculation. This standard orientation is chosen to optimize performance. Then, one can find information 
about how many basis functions and how many primitive gaussians are used in the calculation. There is also a line which indicates the predicted energy computed by the single point calculation. It also indicates the values of the convergence criteria in the self-consistent field (SCF) iteration process. A Mulliken population analysis follows the SCF energy results. This analysis partitions the total charge among the atoms in the molecule. Mulliken population analysis is an arbitrary scheme for assigning charges because atomic charges, unlike the electron density, are not a quantum mechanical observable and are not unambiguously predictable from the first principle. The section labeled Total atomic charges indicates the estimated total charge on each atom in the molecule. A very important section of the output consists of the molecular orbital (MO) coefficients and orbital energies. The atomic orbital contributions for each atom in the cluster are given for each molecular orbital, numbered in order of increasing energy. The MO's energy is given in the row labeled EIGENVALUES preceding the orbital coefficients. The symmetry of the orbital and whether it is an occupied orbital or a virtual (unoccupied) orbital appears immediately under the orbital number. As part of the population analysis, Gaussian computes the isotropic hyperfine coupling constants. These values are given for each atom in the cluster and they are found in the section labeled "Fermi contact analysis". This is one of the most useful results from the calculation since it can be compared with EPR results. The values for the isotropic Fermi coupling constant are given in atomic units but also in $\mathrm{MHz}$, Gauss, and wavenumber. Thus, direct comparison with experiment is straightforward.

GaussView is a graphical user interface designed to be used with Gaussian. It can also perform Gaussian calculations and build molecules in a three dimensional picture. Results that can be viewed include optimized molecular structures, molecular orbitals, electron density surfaces, and others. Through its visualization facility, this program allows the operator to rotate, translate and zoom in on the molecules. The structures that I will show in the next section are calculated with Gaussian 98 and visualized with the help of the GaussView. 


\subsection{Results of the calculations and comparison with experimental data}

The results of the Gaussian and GaussView calculations for several point defects in $\mathrm{KH}_{2} \mathrm{PO}_{4}$ are presented here and a comparison with the experimental data from electron paramagnetic resonance measurements is discussed in some detail. The primary results from the calculations in which I am especially interested are the minimum energy, the isotropic Fermi contact coupling terms, and the lattice relaxation that takes place when a point defect is present in the lattice.

The lattice structure of the perfect KDP crystal (no point defects) has been described in Chapter 3. Here, with the help of Gaussian and GaussView programs, I have simulated and pictured this perfect structure for a cluster of 41 atoms. The cluster contains five $\mathrm{PO}_{4}$ units connected by hydrogen atoms located at the average distance between two oxygen atoms. To simulate the perfect structure (without point defects), the group of atoms chosen is $\mathrm{H}_{16}{ }^{1+} \mathrm{P}_{5}{ }^{5+} \mathrm{O}_{20}{ }^{2-}$ with total valence charge +1 . In this ionic picture, there are a total of 250 electrons (no electrons from the protons, ten electrons from each phosphorus ion, and ten electrons from each oxygen ion) in this cluster. There is no unpaired spin because of the even number of electrons, and the spin multiplicity is 1 . The cluster is a closed shell system and the restricted Hartree-Fock model is appropriate for this calculation. The chosen basis set is $6-31 \mathrm{G}$ and no geometry optimization was requested. The configuration chosen for this preliminary calculation was used for setting up initial conditions for subsequent defectrelated calculations. The Gaussian calculation took approximately 20 minutes and the result represents the crystalline structure of KDP. This is shown in Figure 7.1. The view has the $c$ axis coming out of the paper. All atoms have symbols and labels. They are numbered from 1 starting at the center phosphorous atom. The central $\mathrm{PO}_{4}$ unit is labeled from 1 to 5 and the surrounding hydrogen atoms are from 6 to 9. The phosphorous 10 and 18 and their bonded oxygen atoms are above the plane of the paper and phosphorous 26 and 34 together with their bonded oxygen atoms are below. The plane of the paper contains the $\mathrm{P} 1$ atom. The $\mathrm{O} 2$ and 
$\mathrm{O} 3$ atoms are above and the $\mathrm{O} 4$ and $\mathrm{O} 5$ atoms are below this plane.

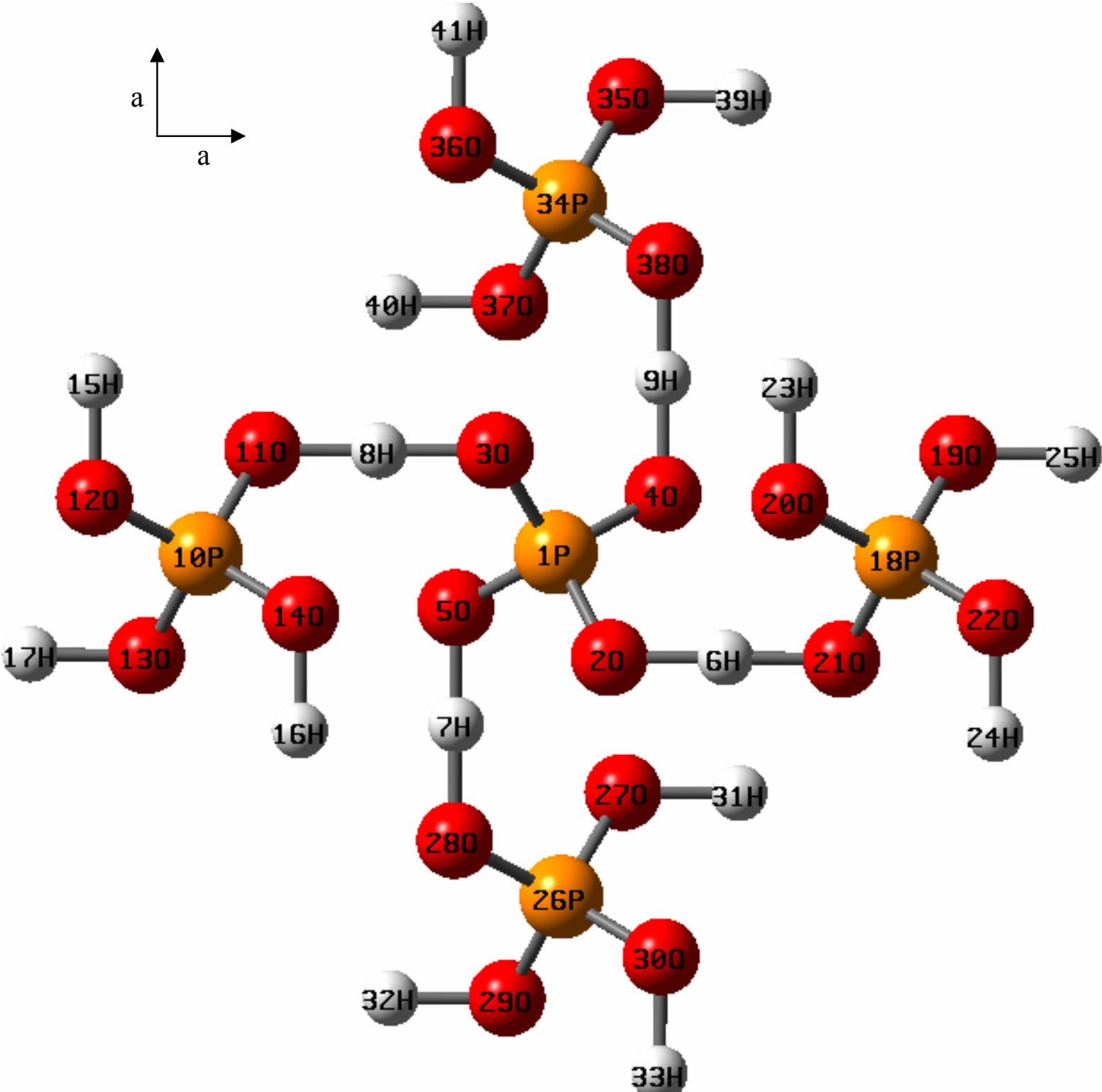

Figure 7.1. A 3D view of the $\left(\mathrm{H}_{16} \mathrm{P}_{5} \mathrm{O}_{20}\right)$ cluster. The $\mathrm{c}$ axis is perpendicular to the plane of the paper. Phosphorous 1 (P1) is in the plane of the paper, whereas $\mathrm{P} 10$ and $\mathrm{P} 18$ are above this plane and $\mathrm{P} 26$ and $\mathrm{P} 34$ are below. In the central $\mathrm{PO}_{4}$ unit, $\mathrm{O} 2$ and $\mathrm{O} 3$ are above $\mathrm{P} 1$ and $\mathrm{O} 4$ and $\mathrm{O} 5$ are below. 
Rotating this cluster of atoms by 90 degrees about the $a$-axis contained in the paper plane, we obtain Figure 7.2. This is the same structure, only with a different orientation relative to the page. In Figure 7.2, the plane of the paper contains the c axis and one $a$-axis. The other $a$-axis is perpendicular to the plane of the paper. Due to the tetragonal symmetry of the crystal, in this orientation, the $\mathrm{PO}_{4}$ units that contain P26 and P34 at their centers appear superimposed and only P26 can be seen. For the same reason, several other atoms are aligned and become overlaid in this picture. For example, O28, $\mathrm{H} 7, \mathrm{O} 5, \mathrm{O} 36$, and $\mathrm{H} 41$ as well as $\mathrm{H} 33$, O30, O4, H9, and $\mathrm{O} 38$ would form a straight line if bonded. The total energy computed in the calculation for this structure is $\mathrm{E}=-3208.212$ Hartrees or $-117.899 \mathrm{eV}(1$ Hartree $=27.212 \mathrm{eV})$

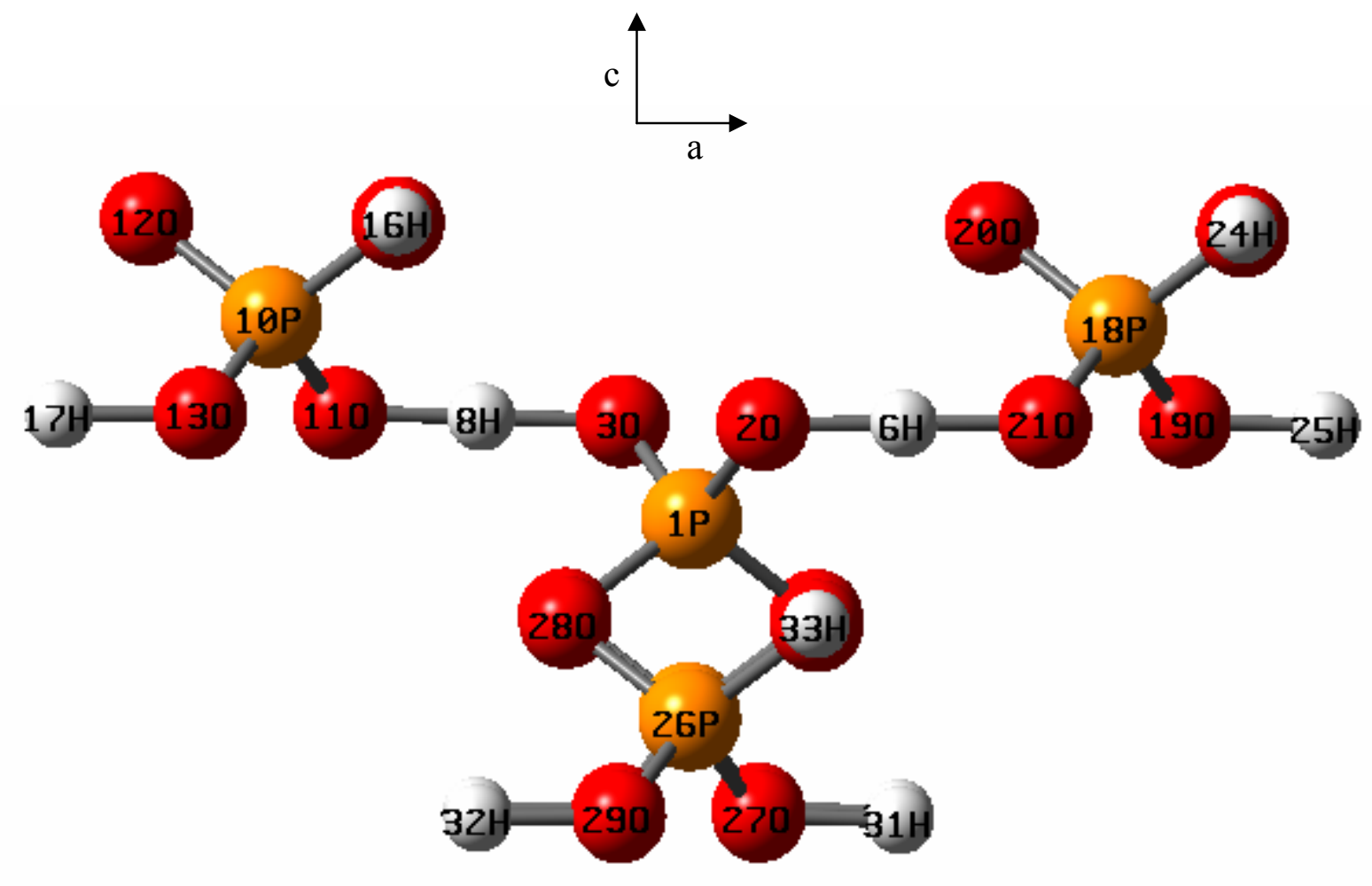

Figure 7.2. KDP tetragonal structure. In this 3D view one $a$ axis is perpendicular to the plane of the paper. The other $a$ axis and $c$ axis are in the plane of the paper. $\mathrm{PO}_{4}$ units having P26 and P34 in the center are superimposed in this view due to the symmetry of the crystal. 
In order to model the various point defects in the KDP lattice, we have used the same cluster of atoms but we made several changes in the input of the Gaussian calculation such as charge, spin multiplicity, and others, that are specific for each defect. The self trapped hole $\left(\mathrm{H}_{2} \mathrm{PO}_{4}\right)^{0}$ center in KDP is one of the first holelike centers to be reported in the literature, and its well-established model is a hole equally shared by two oxygen ions on one $\mathrm{PO}_{4}$ unit in the otherwise perfect lattice. From EPR measurements [60], it is known that in addition to the characteristic $31 \mathrm{G}$ phosphorus hyperfine splitting, the $\left(\mathrm{H}_{2} \mathrm{PO}_{4}\right)^{0}$ center also has a resolved hyperfine pattern due to the two nearest-neighbor hydrogen ions (protons). The hyperfine interaction with the two hydrogen ions gives a triplet EPR spectrum with $3.2 \mathrm{G}$ spacings between adjacent lines. This experimental result is further elaborated by the angular dependence of the EPR signal and ENDOR data. In a crystal of DKDP, the EPR measurements [62] at low temperature gave an interesting result that one of the principal $g$ value directions is very nearly along the line joining the $\mathrm{O} 2$ and $\mathrm{O} 3$ oxygens. At low temperature, the crystal has an orthorhombic structure and these oxygen atoms are situated further away from the phosphorus atom than the other two oxygens from the same $\mathrm{PO}_{4}$ unit. The self trapped hole is shared by the two oxygen atoms located further away from the phosphorus. This is a very important result for our calculations even though the EPR signal is unstable at room temperature. However, this result implies that in a calculation of this defect in the room temperature structure, one would expect to discover that the oxygen atoms sharing the hole would relax further away from the phosphorus in similarity to the EPR data.

In the process of modeling the self trapped hole center in KDP, we have used the previous cluster of 41 atoms $\left(\mathrm{H}_{16} \mathrm{P}_{5} \mathrm{O}_{20}\right)$, with the "addition" of one hole. This changes the valence charge from $1+$ to $2+$ and the spin multiplicity from 1 to 2 since the total spin is now $\mathrm{S}=1 / 2$. We have used the same basis set (6-31G) but now invoke the unrestricted HartreeFock theory model because the system is open shell. A summary of the Gaussian results from all the calculations performed for the self-trapped hole center is presented in Table 7.1 
on page 108. This table contains information about the ions that were allowed to move during the calculation, the total energy at the end of the calculation, the Fermi contact terms for P1, H6, and H8, the basis set used, and the processing time. The first entry in Table 7.1 shows results from a short calculation for which all the atoms were fixed at their regular positions. No geometry optimization was performed and the Fermi contact values are small for the majority of the atoms except for the four oxygen and hydrogen atoms surrounding the central phosphorus (P1) which came out equal and fairly large. These results do not agree with experiment.

After this, a geometry optimization calculation was performed and the coordinates of $\mathrm{O} 2, \mathrm{O} 3, \mathrm{H} 6$, and $\mathrm{H} 8$ atoms were free to change during the calculation while all the other atoms were frozen. $\mathrm{P} 1$ is the central phosphorus atom (Figure 7.1), $\mathrm{O} 2$ and $\mathrm{O} 3$ are the two oxygens located above the plane of the paper, and $\mathrm{H} 6$ and $\mathrm{H} 8$ are the protons bonded to these two oxygen ions. The second row in Table 7.2 contains several features of this calculation. More details about these results will be given in the following, but first I will discuss the input for the geometry optimization.

The atom coordinates used in the input are the ones corresponding to the tetragonal structure of KDP and are given below. When we look at the "Molecule specifications" and "Variables" columns, we can observe two important features. First is that the O4 and O5

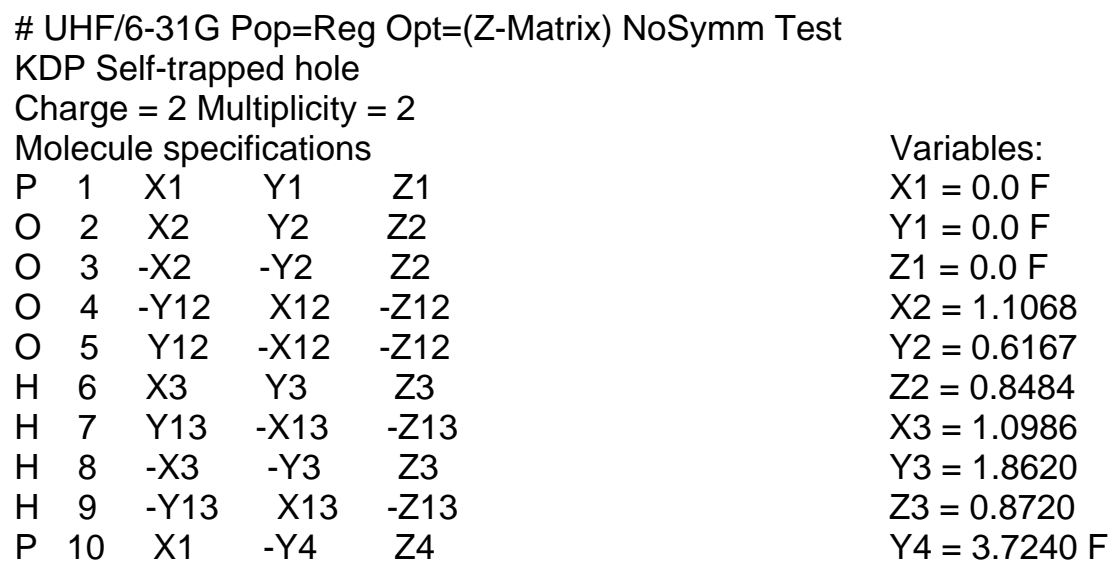




\begin{tabular}{|c|c|c|c|c|c|}
\hline $\mathrm{O}$ & 11 & $-X 12$ & $-Y 5$ & Z5 & $\mathrm{Z} 4=1.7440 \mathrm{~F}$ \\
\hline $\mathrm{O}$ & 12 & $-Y 12$ & $-Y 6$ & Z6 & $Y 5=3.1073 \mathrm{~F}$ \\
\hline $\mathrm{O}$ & 13 & X12 & $-Y 7$ & Z5 & $Z 5=0.8958 \mathrm{~F}$ \\
\hline $\mathrm{O}$ & 14 & Y12 & $-Y 8$ & Z6 & $Y 6=4.831 \mathrm{~F}$ \\
\hline $\mathrm{H}$ & 15 & -Y13 & -Y9 & Z9 & $Z 6=2.5926 \mathrm{~F}$ \\
\hline $\mathrm{H}$ & 16 & Y13 & $-Y 10$ & Z9 & $Y 7=4.341 \mathrm{~F}$ \\
\hline $\mathrm{H}$ & 17 & X13 & $-Y 11$ & Z13 & $Y 8=2.617 \mathrm{~F}$ \\
\hline $\mathrm{O}$ & 19 & $-\mathrm{X} 12$ & Y7 & Z5 & $Y 9=4.831 F$ \\
\hline $\mathrm{O}$ & 20 & -Y12 & Y8 & Z6 & $Z 9=2.616 \mathrm{~F}$ \\
\hline $\mathrm{O}$ & 21 & X12 & Y5 & Z5 & $\mathrm{Y} 10=2.617 \mathrm{~F}$ \\
\hline O & 22 & Y12 & Y6 & Z6 & $\mathrm{Y} 11=5.586 \mathrm{~F}$ \\
\hline $\mathrm{H}$ & 23 & $-Y 13$ & Y10 & Z9 & $\mathrm{X} 12=1.1068 \mathrm{~F}$ \\
\hline $\mathrm{H}$ & 24 & Y13 & Y9 & Z9 & $\mathrm{Y} 12=0.6167 \mathrm{~F}$ \\
\hline $\mathrm{H}$ & 25 & $-\mathrm{X} 13$ & Y11 & Z13 & $\mathrm{Z} 12=0.8484 \mathrm{~F}$ \\
\hline $\mathrm{P}$ & 26 & Y4 & $\mathrm{X} 1$ & $-Z 4$ & $\mathrm{X} 13=1.0986 \mathrm{~F}$ \\
\hline O & 27 & Y8 & Y12 & $-Z 6$ & $\mathrm{Y} 13=1.862 \mathrm{~F}$ \\
\hline O & 28 & Y5 & $-\mathrm{X} 12$ & $-Z 5$ & $\mathrm{Z} 13=0.872 \mathrm{~F}$ \\
\hline $\mathrm{O}$ & 29 & Y6 & -Y12 & $-Z 6$ & \\
\hline O & 30 & Y7 & $\mathrm{X} 12$ & $-Z 5$ & \\
\hline $\mathrm{H}$ & 31 & Y10 & Y13 & $-Z 9$ & \\
\hline $\mathrm{H}$ & 32 & Y9 & $-Y 13$ & $-Z 9$ & \\
\hline $\mathrm{H}$ & 33 & Y11 & $\mathrm{X} 13$ & $-\mathrm{Z13}$ & \\
\hline $\mathrm{P}$ & 34 & $-Y 4$ & $\mathrm{X} 1$ & $-Z 4$ & \\
\hline O & 35 & $-Y 6$ & Y12 & $-Z 6$ & \\
\hline $\mathrm{O}$ & 36 & $-Y 7$ & $-\mathrm{X} 12$ & $-\mathrm{Z5}$ & \\
\hline O & 37 & $-Y 8$ & -Y12 & $-Z 6$ & \\
\hline O & 38 & $-Y 5$ & X12 & $-Z 5$ & \\
\hline $\mathrm{H}$ & 39 & $-Y 9$ & Y13 & $-Z 9$ & \\
\hline $\mathrm{H}$ & 40 & -Y10 & -Y13 & $-Z 9$ & \\
\hline $\mathrm{H}$ & 41 & -Y11 & -X13 & -Z3 & \\
\hline
\end{tabular}

atoms have different coordinate symbols than $\mathrm{O} 2$ and $\mathrm{O} 3$ atoms even though they have the same coordinates in absolute value before the calculation begins.

This difference in notation is necessary because they will be treated differently, in other words, $\mathrm{O} 4$ and $\mathrm{O} 5$ are not allowed to move during the optimization whereas $\mathrm{O} 2$ and $\mathrm{O} 3$ are allowed to move during the calculation. The same holds true for the coordinates of $\mathrm{H} 7$ and $\mathrm{H} 9$ which are the same in absolute value as those of $\mathrm{H} 6$ and $\mathrm{H} 8$ before the calculation, but $\mathrm{H} 7$ and $\mathrm{H} 9$ are fixed during the calculation. Second, the variables $\mathrm{X} 2, \mathrm{Y} 2, \mathrm{Z} 2$ of $\mathrm{O} 2$ and $\mathrm{O} 3$, and also $\mathrm{X} 3, \mathrm{Y} 3, \mathrm{Z} 3$ of $\mathrm{H} 6$ and $\mathrm{H} 8$ do not have the "frozen" symbol since these atoms are allowed to relax during the geometry optimization.

This geometry optimization concluded after more than six hours with the minimum energy equal to $\mathrm{E}=-3207.850$ Hartree or $-117.885 \mathrm{eV}$ which means an increase of the total 
energy of $14 \mathrm{meV}$ with the addition of the hole in the cluster. During this calculation, the minimum energy was acquired with the relaxation of the two oxygens which moved further away from the phosphorus by $0.12 \AA$ while the angle O2'-P1'-O3' decreased by approximately 10 degrees, making the new distance $\mathrm{O} 2$ '-O3' to increase only by $0.05 \AA$. Also the hydrogen atoms H6 and H8 moved during the calculation. They both moved further away from their bonded oxygen atoms by $0.3 \AA$, and the angles P1-O2-H6 and P1-O3-H8 have increased by about 1 degree. These changes are illustrated in Figure 7.3 which is a three-dimensional representation of the $\mathrm{PO}_{4}$ central unit where the self-trapped hole is localized. I show, in this figure, the ions before the calculation (using the original notation) and the ions after the calculation (with primes). This view is similar to that presented in Figure 7.2. To better see the new positions, I have rotated the $\mathrm{PO}_{4}$ unit only a few degrees off the $c$ axis and approximately 45 degrees off the $a$ axis.

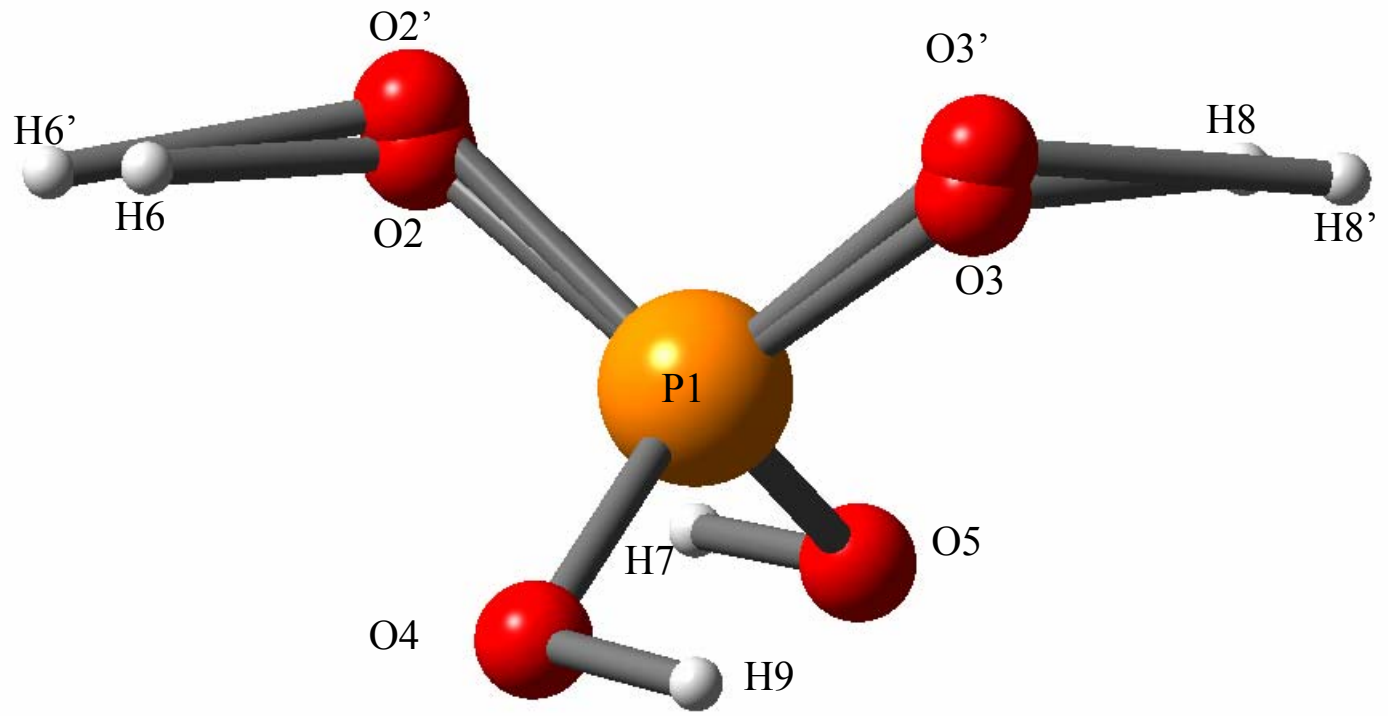

Figure 7.3. A three-dimension view of the central $\mathrm{PO}_{4}$ unit with the adjacent hydrogen atoms showing the change in the position of the four atoms $\mathrm{O} 2$, O3, H6, and $\mathrm{H} 8$ after the geometry optimization. O2', O3', H6', and H8' represent the atoms at the new positions after the geometry optimization. 
The most helpful features emerging from our study that permit direct comparison with the experimental data are the relative values of the isotropic Fermi contact coupling terms produced as a result of the geometry optimization. The Fermi contact term represents a measure of the isotropic hyperfine interaction (the interaction energy between the electron and the nucleus). The origin of the isotropic hyperfine interaction comes from the $s-$ like orbitals containing unpaired spin density. The $\mathrm{n} s$ electron density at the nucleus is nonzero and this finite density gives rise to the isotropic hyperfine interaction. The Hamiltonian operator for the hyperfine interaction may be obtained from the Fermi isotropic interaction energy for a system with one electron $\quad W_{i s o}=-\frac{8 \pi}{3}|\psi(0)|^{2} \mu_{e_{z}} \mu_{N_{z}} \quad$ by replacing the magnetic moments with their corresponding operators $\hat{\mu}_{e_{z}}=-g \beta \hat{S}_{z}$ and $\hat{\mu}_{N_{z}}=g_{N} \beta_{N} \hat{I}_{z}$. The corresponding Hamiltonian can be written, in a short form, as $\hat{\mathcal{H}}_{i s o}=h A_{0} \hat{S}_{z} \hat{I}_{z}$ with $A_{0}$ called the isotropic hyperfine coupling constant (measured in Hertz), and $h A_{0}$ the interaction energy between the electron and the nucleus. In an electron paramagnetic resonance experiment, the hyperfine constant is determined by fitting the angular dependence data to a Hamiltonian containing an electron Zeeman term and a hyperfine term. A least-squares fitting procedure is used to obtain the best-fit values of the $g$ and $A$ matrix parameters.

The second row in Table 7.2 representing the results from the geometry optimization gives very good agreement with the experimental EPR data reported by Stevens et al. in 1999 [62] and described on page 103. Although not measured, the isotropic Fermi contact terms calculated for oxygen ions $\mathrm{O} 2$ and $\mathrm{O} 3$ (Figure 7.3) are approximately 41 Gauss, and this compares with 9 Gauss calculated for oxygen ions $\mathrm{O} 4$ and O5. The hyperfine interactions calculated for the central phosphorus ion P1 and for the hydrogen ions H6 and H8 are very close to the values extracted from the EPR measurements. The Fermi contact values for all the other atoms in the cluster are very small, approximately 1 Gauss or less. These results confirm the model that the hole is mostly localized on and equally shared by two oxygen ions and the dominant observed hyperfine interactions are with the phosphorus nucleus and the 
two protons bonded to the oxygen ions that share the hole.

Table 7.2. Summary of Gaussian results for the self-trapped hole center in an $\mathrm{H}_{16} \mathrm{P}_{5} \mathrm{O}_{20}$ cluster. For comparison purposes, the experimental results give a Fermi contact value of approximately $30 \mathrm{G}$ for the primary phosphorus hyperfine interaction and $3.2 \mathrm{G}$ for the hyperfine interaction with one proton.

\begin{tabular}{|c|c|c|c|c|c|c|c|}
\hline \multirow{2}{*}{$\begin{array}{l}\text { Geometry- } \\
\text { Optimized? }\end{array}$} & \multirow{2}{*}{$\begin{array}{l}\text { Ions } \\
\text { Moved }\end{array}$} & \multirow{2}{*}{$\begin{array}{c}\text { Total } \\
\text { Energy } \\
\text { (Hartrees) }\end{array}$} & \multicolumn{3}{|c|}{ Fermi Contact (Gauss) } & \multirow{2}{*}{$\begin{array}{l}\text { Basis Set } \\
\text { /Theory }\end{array}$} & \multirow{2}{*}{$\begin{array}{l}\text { CPU } \\
\text { Time }\end{array}$} \\
\hline & & & P1 & H6 & H8 & & \\
\hline No & None & -3207.760 & -45 & -13 & -13 & $\begin{array}{l}\text { 6-31G } \\
\text { /UHF }\end{array}$ & $5 \mathrm{~min}$ \\
\hline Yes & $\begin{array}{l}\mathrm{O} 2, \mathrm{O} 3, \\
\mathrm{H} 6, \mathrm{H} 8\end{array}$ & -3207.850 & -52 & -5 & -5 & $\begin{array}{l}\text { 6-31G } \\
\text { /UHF }\end{array}$ & $\sim 6 \mathrm{hr}$ \\
\hline Yes & $\begin{array}{l}\mathrm{O} 2, \mathrm{O} 3, \\
\mathrm{H} 6, \mathrm{H} 8\end{array}$ & -3169.137 & -273 & -4 & -4 & $\begin{array}{l}\text { STO-3G } \\
\text { /UHF }\end{array}$ & $\sim 1 \mathrm{hr}$ \\
\hline Yes & $\begin{array}{c}\mathrm{P} 1, \mathrm{O} 2, \mathrm{O} 3, \\
\mathrm{H} 6, \mathrm{H} 8\end{array}$ & -3169.145 & -306 & -3 & -3 & $\begin{array}{l}\text { STO-3G } \\
\text { /UHF }\end{array}$ & $\sim 1 \mathrm{hr}$ \\
\hline Yes & $\begin{array}{l}\mathrm{O} 2, \mathrm{O} 3, \\
\mathrm{H} 6, \mathrm{H} 8\end{array}$ & -3209.587 & -46 & -5 & -5 & $\begin{array}{c}6-31++\mathrm{G}(\mathrm{d}, \mathrm{p}) \\
/ \mathrm{UHF}\end{array}$ & $\begin{array}{c}\sim 7 \\
\text { days }\end{array}$ \\
\hline Yes & $\begin{array}{l}\mathrm{O} 2, \mathrm{O} 3, \\
\mathrm{H} 6, \mathrm{H} 8\end{array}$ & -3207.837 & -59 & -10 & -10 & $\begin{array}{l}\text { 6-31G } \\
\text { /UMP2 }\end{array}$ & $\begin{array}{c}11 \\
\text { days }\end{array}$ \\
\hline
\end{tabular}


The next entries in the Table 7.2 represent results from various calculations done on the same cluster and same defect but using a different basis set or theoretical model. For example, rows three and four represent results from calculations done with another basis set. STO-3G is a smaller basis set than 6-31G, containing only 161 basis functions. Therefore the calculation is much shorter, but the result is not in very good agreement with the experiment. Allowing P1 to also move during the geometry optimization did not improve the results. The last two entries in the table refer to calculations which required a much longer time. In the next to last entry, a larger basis set was used $(6-31++G(d, p))$ and the number of basis function increased to 591. In the last entry, a different method of calculation (unrestricted Møller-Plesset, keyword UMP2) was used. This latter approach is a correction to the Hartree-Fock method that accounts for electron correlations effects. In general, the use of this method will lead to a more pronounced delocalization of the hole [72], which is shown in our calculation and this result gives more confidence to the accepted model that the hole is localized on two oxygen ions.

The purpose of all the calculations described above is to show that it is not just any combination of theoretical model, molecular configuration, and basis set that would give reasonable results and agreement with experiment. Thus, it is not a matter of guessing but of choosing the right cluster and constructing the correct input. From the series of calculations for the self-trapped hole center it appears that the combination of the cluster $\mathrm{H}_{16} \mathrm{P}_{5} \mathrm{O}_{20}$ with Hartree-Fock model, and with the basis set 6-31G gives very good agreement with experimental data and reasonable calculation time. Therefore, the next calculations performed on the other defects will use this combination of input specifications.

The next point defect for which Gaussian calculations were performed is the proton vacancy hole center, labeled $\left(\mathrm{HPO}_{4}\right)^{-}$. From EPR experiments, we know that the $\left(\mathrm{HPO}_{4}\right)^{-}$ center has a spectrum composed of a pair of lines separated by 31 Gauss and with a g value of 2.0217 when the magnetic field is parallel to $c$-axis [60]. The $31 \mathrm{G}$ separation arises from 
a phosphorus hyperfine interaction $\left({ }^{31} \mathrm{P}\right.$ is $100 \%$ abundant with $\left.\mathrm{I}=1 / 2\right)$. The proposed model for this center is a hole trapped on a single oxygen ion adjacent to a hydrogen vacancy [5861]. In order to calculate the isotropic Fermi contact terms for this defect, we have constructed the following input. The new cluster is $\mathrm{H}_{15} \mathrm{P}_{5} \mathrm{O}_{20}$ because of the exclusion of one proton (hydrogen ion). Arbitrarily, we select H6 as the missing proton. The H6 proton is the proton that is bonded to $\mathrm{O} 2$. With the exclusion of one proton the cluster becomes neutral. However, in order to reproduce the hole trap we must set the total charge +1 . With the addition of the hole, the spin becomes $1 / 2$. The geometry optimization was performed with a 6-31G basis set and the unrestricted Hartree-Fock theoretical model.

From a preliminary calculation for which all atoms were fixed, the results show that the minimum energy is -3207.549 Hartree and the isotropic Fermi contact terms are approximately $-60 \mathrm{G}$ for $\mathrm{P} 1$ and $-52 \mathrm{G}$ for $\mathrm{O} 2$. All the other Fermi contact terms are relatively very small compared with these two values, with the only exceptions from this being $\mathrm{O} 21$ and $\mathrm{P} 18$ (Figure 7.2). The Fermi contact term for $\mathrm{O} 21$ is $-9 \mathrm{G}$ and for P18 is $-3 \mathrm{G}$. These ions are located at the nearest distance from where the missing proton used to be. This initial outcome leads to the conclusion that the hole has maximum interaction with $\mathrm{O} 2$ and $\mathrm{P} 1$ and only very weak interaction with $\mathrm{O} 21$ and P18. Next step in the calculation process is to allow all four atoms $\mathrm{P} 1, \mathrm{O} 2, \mathrm{P} 18$, and $\mathrm{O} 21$ to relax during the geometry optimization. The result is that the hyperfine interaction (in the form of the Fermi contact term) with P1 increases while the interactions with the other atoms decrease. The minimum energy has a small decrease to $-3207.586 \mathrm{H}$ after the relaxation of the four atoms to the new positions. Also, the distances between these atoms slightly decrease. From a distance of $2.63 \AA$ used in the first calculation, the $\mathrm{O} 2$ and $\mathrm{O} 21$ ions have moved closer and are now $2.48 \AA$ apart. The distance between P1 and P18 decreased a little from $4.11 \AA$ to $4.09 \AA$. This is an important result showing that even though the symmetry of the lattice may suggest the sharing of the hole by the two oxygen ions (O2 and $\mathrm{O} 21)$ having hyperfine interaction with two phosphorus 
atoms (P1 and P18), the EPR experiment and the modeling give strong evidence that the hole is primarily localized only on one oxygen.

Since the results from my calculation confirm that the hole is trapped on $\mathrm{O} 2$ with a large Fermi contact with $\mathrm{P} 1$, the next step is to let the $\mathrm{O} 2$ and $\mathrm{P} 1$ atoms relax during a geometry optimization. The result is that the Fermi contact terms changed in absolute value, to $-68 \mathrm{G}$ for $\mathrm{P} 1$ and $-48 \mathrm{G}$ for $\mathrm{O} 2$, and the two atoms move away one from each other. The distance between them increases from $1.52 \AA$ to $1.73 \AA$ with $\mathrm{P} 1$ changing its coordinates very little (a maximum change of $0.045 \AA$ in the y coordinate and a minimum change of $0.014 \AA$ in the $\mathrm{x}$ coordinate). To illustrate the change in the geometry of the central $\mathrm{PO}_{4}$ unit during the geometry optimization, in Figure $7.4 \mathrm{I}$ present an overlap of the $\mathrm{PO}_{4}$ units before and after the calculation. In this orientation of the three-dimensional view, one of the $a$ axis

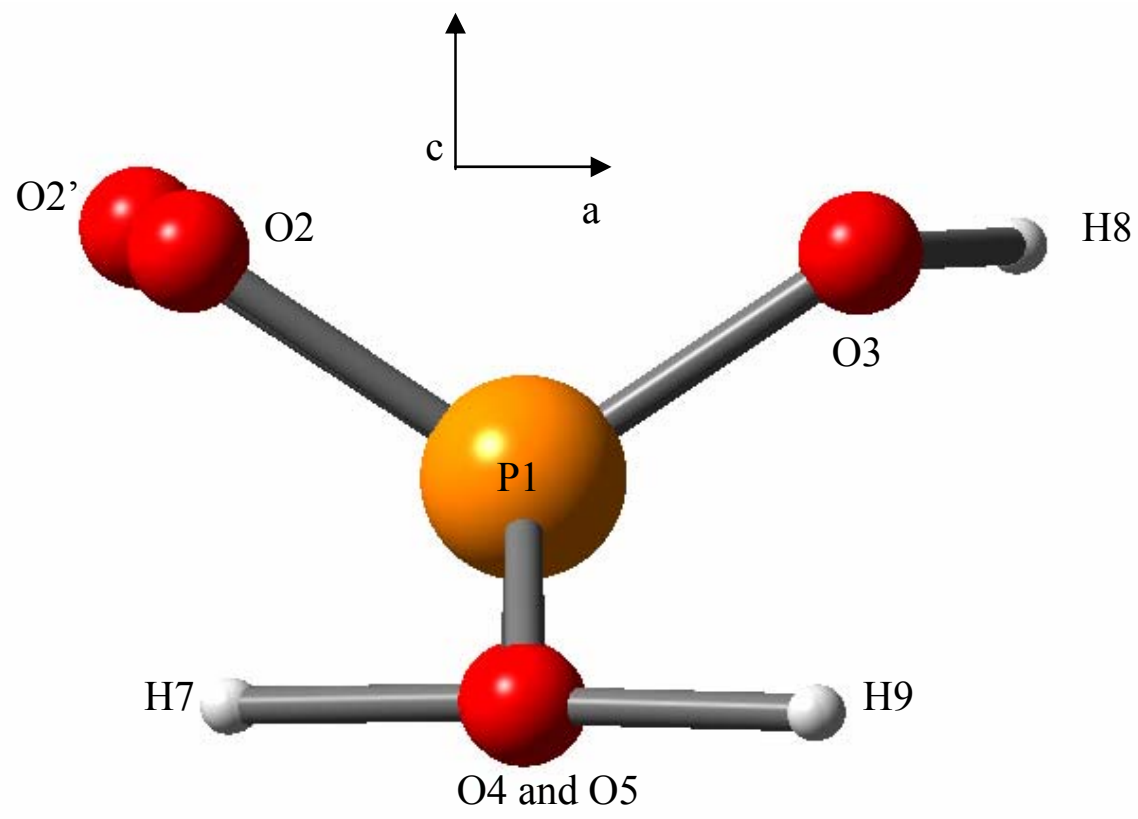

Figure 7.4. A three-dimensional view of the central $\mathrm{PO}_{4}$ unit missing one hydrogen atom in order to model the proton vacancy point defect. This is a before and after the geometry optimization representation. The atoms at the new positions are labeled with primes. 
is perpendicular to the plane of the paper and the $c$ axis is almost vertical in the plane of the paper. For this orientation, the $\mathrm{O} 4$ and $\mathrm{O} 5$ atoms appear overlaid. From the relaxation of the cluster, the minimum energy becomes -3207.587 Hartree, a decrease of 0.038 Hartree from the "fixed"-atoms structure calculated initially. The next geometry optimization performed allowed only the $\mathrm{O} 2$ atom to move while keeping all the other atoms fixed. The results are that during the geometry optimization, in order to achieve the minimum energy of -3207.582 Hartree, O2 moved away from P1 by approximately $0.22 \AA$. The isotropic Fermi terms are $64 \mathrm{G}$ for $\mathrm{P} 1$ and $-49 \mathrm{G}$ for $\mathrm{O} 2$. The Fermi terms for the other three oxygen ions surrounding P1 are in average $-1.5 \mathrm{G}$. Even though the value for the Fermi contact term for P1 is about two times larger compared with the EPR measurements, we still consider the Gaussian results very good for the major reason that our calculated model shows the hole essentially being trapped by a single oxygen ion and primarily having hyperfine interaction only one phosphorus ion. Thus, we can say that for this point defect we have obtained very good qualitative results.

The last hole center for which I have done calculations is the Si-hole center, labeled $\left(\mathrm{H}_{2} \mathrm{SiO}_{4}\right)^{-}$. This point defect is produced by ionizing radiation, either $\mathrm{x}$ rays or intense 266$\mathrm{nm}$ pulsed laser beam, at $77 \mathrm{~K}$. The EPR spectrum is best resolved at room temperature, with the c-axis of the KDP crystal parallel to the applied magnetic field. The spectrum consists mainly of a pair of lines separated by 7.1 Gauss and a much weaker doublet with $10 \mathrm{G}$ splitting centered on each of the primary lines. The model for this point defect is predicted by the angular dependence data of its EPR signal [69] and consists of a hole trapped at one oxygen ion adjacent to a silicon impurity substituting for a phosphorus ion. The pair of lines separated by $7.1 \mathrm{G}$ is due to a hyperfine interaction with one proton and the $10 \mathrm{G}$ splitting is due to the ${ }^{29} \mathrm{Si}$ nucleus $(4.7 \%$ abundant, $I=1 / 2)$. When a silicon ion $\left(\mathrm{Si}^{4+}\right)$ substitutes for a phosphorus ion $\left(\mathrm{P}^{5+}\right)$ in KDP, an extra positive charge is needed to maintain electrical neutrality in the crystal. This is most easily accomplished by trapping a hole on an oxygen 
ion adjacent to the silicon impurity.

To model the Si-hole center and compute the Fermi contact terms, I have chosen the same original cluster except that the central phosphorus P1 is replaced with a silicon ion, labeled Si1. With this change, the new cluster is $\mathrm{H}_{16} \mathrm{SiP}_{4} \mathrm{O}_{20}$. The total charge of this cluster is +1 with the addition of the hole. The spin is $1 / 2$ and the calculations are performed using unrestricted Hartree-Fock theory with the basis set 6-31G. From the initial calculation with no geometry optimization, the minimum energy came out to be -3156.412 Hartree and the Fermi terms indicating the isotropic hyperfine interaction are $-53 \mathrm{G}$ for $\mathrm{O} 2,13 \mathrm{G}$ for $\mathrm{Si} 1$ and $-6 \mathrm{G}$ for H6. These results can be straightforwardly interpreted as a hole localized primarily on one oxygen ion $(\mathrm{O} 2)$ that has hyperfine interaction with silicon ion (Sil) and with one proton (H6). Even without the geometry optimization, which usually improves results, the above numbers are in very good qualitative and quantitative agreement with the hyperfine values from the EPR experiment. However, for the consistency of this study, we have performed a geometry optimization with $\mathrm{Si1}, \mathrm{O} 2$ and $\mathrm{H} 6$ free to move. The new Fermi contact terms are given in Table 7.3. The minimum energy decreased to -3156.435 Hartree.

Table 7.3. Fermi contact coupling terms resulted from the geometry optimization of the Si-hole center.

\begin{tabular}{cc}
\hline Atom & Fermi Contact (Gauss) \\
\hline Si1 & 9.57 \\
\hline O2 & -55.54 \\
\hline O3 & -0.94 \\
\hline O4 & -4.15 \\
\hline O5 & -0.27 \\
\hline H6 & -8.71 \\
\hline O21 & -8.61 \\
\hline
\end{tabular}


Lattice relaxation is small, with a tendency towards increased distances between the atoms. Figure 7.5 shows these changes in the positions of the atoms within the central $\mathrm{SiO}_{4}$ unit. Highlighted atoms are Sil', O2', and H6' at the new positions generated by the geometry optimization. These three atoms, at the new positions, are also labeled 1, 2, and 3 in this same order. Figure 7.5 represents two $\mathrm{SiO}_{4}$ units interpenetrated. Because of the small changes in the coordinates of the Sil ion during the geometry optimization it is hard to distinguish between the new and old positions and in Figure 7.5 it appears there is no change for $\mathrm{Si1}$.

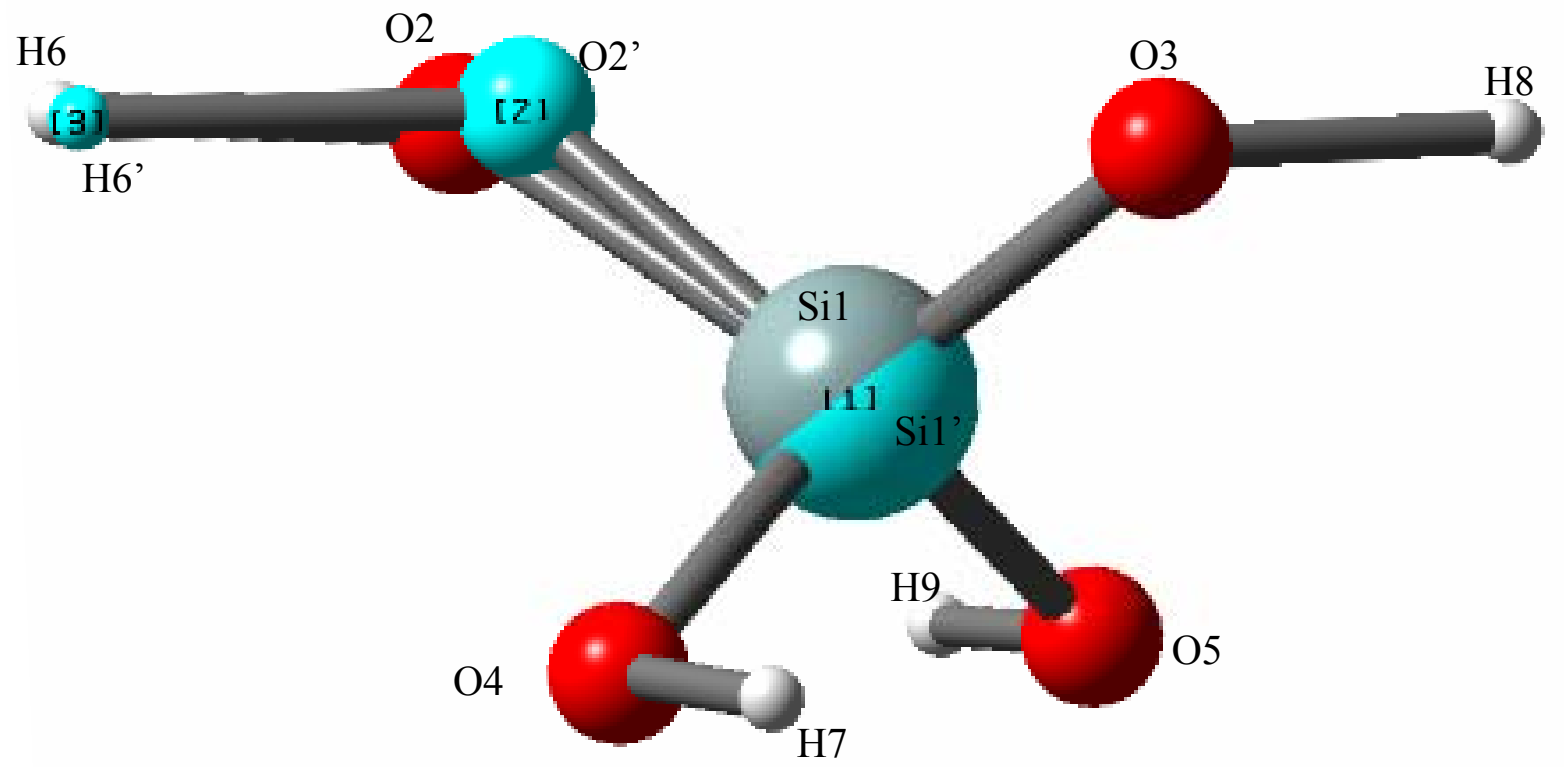

Figure 7.5. A three-dimensional-view of the $\mathrm{SiO}_{4}$ central unit in KDP, showing the "before" and "after" positions of the atoms. 
In the end of this section, I will discuss the electron trap centers in KDP known as oxygen vacancies. Results from EPR experiments will be compared with results from Gaussian calculations. Five electron-like EPR centers can be produced by ionizing radiation and once formed, they are stable for days and even weeks at room temperature [66]. When the magnetic field is parallel to $c$ axis of the crystal, five distinctive, widely split pairs of lines are observed. The proposed model is an oxygen vacancy with an unpaired electron trapped on the neighboring $\mathrm{PO}_{3}$ unit, thus the $\left(\mathrm{PO}_{3}\right)^{2-}$ notation. The large hyperfine interaction with a $100 \%$ abundant, $I=1 / 2$ nucleus is predicted to be due to phosphorus since the observed splitting (greater than $510 \mathrm{G}$ ) is too large for hydrogen. The other $100 \%$ abundant, $I=1 / 2$ nuclei $\left({ }^{19} \mathrm{~F},{ }^{89} \mathrm{Y},{ }^{103} \mathrm{Rh}\right.$, and $\left.{ }^{169} \mathrm{Tm}\right)$ are not expected to be present in any significant quantity in the KDP crystals. This prediction is supported by various investigators who have previously demonstrated that $\left(\mathrm{PO}_{3}\right)^{2-}$ molecular ions have phosphorus hyperfine splittings in the 600-900 G range [66]. The number of observed $\left(\mathrm{PO}_{3}\right)^{2-}$ centers can be explained if a nearby cation vacancy is part of each defect. In the regular room-temperature KDP lattice, each $\left(\mathrm{PO}_{4}\right)^{3-}$ molecule is hydrogen bonded to four other $\left(\mathrm{PO}_{4}\right)^{3-}$ units. Thus, there are four hydrogen sites (and at least two potassium sites) in the near vicinity of a given oxygen ion. An oxygen vacancy formed during growth may be partially charge compensated by a hydrogen or potassium vacancy at one of these close sites. Then, when an electron is trapped at the divacancy during exposure to ionizing radiation, the resulting complex will be neutral. The experimental data of angular dependence for a $\left(\mathrm{PO}_{4}\right)^{3-}$ center were fitted with a spin Hamiltonian and one of the results obtained is the hyperfine matrix for ${ }^{31} \mathrm{P}$ for center $D$ (Table I in [66]). From this result we have extracted the isotropic Fermi contact for ${ }^{31} \mathrm{P}$ being equal to $637 \mathrm{G}$ for the same center.

The calculations performed in order to describe the oxygen vacancy center were done on the modified cluster $\mathrm{H}_{15} \mathrm{P}_{5} \mathrm{O}_{19}$ since one oxygen $(\mathrm{O} 2)$ and the adjacent hydrogen (H6) were excluded. With the addition of an electron, the total charge of the cluster becomes 1+ 
and the spin is $1 / 2$. Initially, a simple calculation, without a geometry optimization and with all atoms fixed, ended with the minimum energy equal to -3132.751 Hartree and with an isotropic Fermi term for P1 of approximately $586 \mathrm{G}$. The next step was to perform a geometry optimization. The coordinates of the three oxygen ions surrounding the central phosphorus were given different notation during the geometry optimization. The results from these two calculations are summarized in Table 7.4. The results from the calculation are very encouraging in accepting the proposed model [66] that the point defect responsible for the EPR spectrum composed of a widely split doublet is an oxygen vacancy.

Table 7.4. Summary of Gaussian results for a $\left(\mathrm{PO}_{3}\right)^{2-}$ center in KDP. For comparison, the isotropic Fermi contact value from EPR experiment is $637 \mathrm{G}$ for the interaction with the phosphorus (in one experimentally observed center).

\begin{tabular}{ccccccc}
\hline \multirow{2}{*}{$\begin{array}{c}\text { Geometry } \\
\text { Optimization }\end{array}$} & Atoms & Minimum & \multicolumn{2}{l}{ Isotropic Fermi Contact Terms (Gauss) } \\
\cline { 4 - 7 } & moved & Energy & & & \\
\cline { 4 - 7 } & & (Hartree) & P1 & O3 & O4 & O5 \\
\hline No & none & -3132.751 & 586 & -23 & -26 & -13 \\
\hline Yes & O3,O4,O5 & -3132.808 & 619 & -18 & -22 & -9 \\
\hline
\end{tabular}




\section{CHAPTER 8}

\section{SUMMARY}

Thermoluminescence (TL), optical absorption, and electron paramagnetic resonance (EPR) experiments have been performed on lithium niobate and lithium tantalate crystals. Combined optical absorption and EPR measurements were used to characterize the production and thermal decay of point defects in undoped single crystals of $\mathrm{KD}_{2} \mathrm{PO}_{4}$. Gaussian 98 and GaussView software were used to model several point defects in $\mathrm{KH}_{2} \mathrm{PO}_{4}$ and results were compared with experimental data of EPR.

A thermoluminescence peak has been observed in $1 \% \mathrm{Mg}$ stoichiometric $\mathrm{LiNbO}_{3}$ crystals. The maximum in the emission occurs at $94 \mathrm{~K}$ and at $440 \mathrm{~nm}$, when the excitation source at $77 \mathrm{~K}$ is either $60-\mathrm{kV}$ x rays, a $325-\mathrm{nm} \mathrm{cw}$ laser, or a $355-\mathrm{nm}$ pulsed laser. Both laser beams have photon energies below the fundamental absorption of the crystals, thus requiring the formation of separated electron and hole traps to occur in these cases via the near-edge charge-transfer absorption band of the $\mathrm{Fe}^{3+}$ ions, present at trace levels in our crystals. The electron and hole traps participating in the radiative recombination process were identified using EPR and near-infrared optical absorption. A crystal was irradiated at $77 \mathrm{~K}$ and EPR spectra were obtained before and after a warming step to $110 \mathrm{~K}$ that replicated the conditions producing the TL peaks. These EPR spectra showed that the responsible centers are a hole localized on an oxygen ion adjacent to a lithium vacancy and an electron trapped on a regular niobium ion (i.e., a $\mathrm{Nb}_{\mathrm{Nb}}{ }^{4+}$ ) with perhaps a $\mathrm{Mg}^{2+}$ ion nearby. The observation of a 1200-nm absorption band induced at low temperature by below-band-gap laser light supported the shallow electron center assignment. From the relative changes observed in the EPR spectra as a result of warming to $110 \mathrm{~K}$, we conclude that the electron centers are the less stable of the two defects, and that the hole centers are thus the recombination site. Specifically, we suggest that the recombination takes place within a 
$\mathrm{NbO}_{6}$ unit containing an oxygen ion that had initially been a trapping site for the hole (these are $\mathrm{NbO}_{6}$ units that have an adjacent lithium vacancy).

In congruently grown $\mathrm{LiTaO}_{3}$ crystals that were previously subjected to lengthy vapor-transport-equilibration (VTE) treatments we have observed two TL peaks, at 94 and 98 K. This post growth process significantly improves the stoichiometry of the crystals by removing lithium vacancies and tantalum antisite defects. In other words, the VTE treatment effectively removes the dominant nonradiative recombination sites from the crystal and allows radiative recombination to be seen. The TL peaks were produced by initially exposing the crystal at $77 \mathrm{~K}$ to either $\mathrm{x}$ rays or a laser beam $(266,325$, and $355 \mathrm{~nm})$ and then heating the crystal rapidly. Both of the TL peaks had a maximum in their emission near 350 nm. Optical absorption and EPR experiments were used to determine which electron and hole traps contribute to the two TL peaks. Our results suggest that the participating holes are initially localized on oxygen ions, either self-trapped or held by an adjacent stabilizing entity, and that the participating electrons are initially trapped at regular tantalum ions as $\mathrm{Ta}_{\mathrm{Ta}}{ }^{4+}$ centers. The TL peaks are then produced when electrons and holes from these traps undergo radiative recombination within $\mathrm{TaO}_{6}$ units.

In conclusion, we propose that the low-temperature light emitting properties of stoichiometric $\mathrm{LiNbO}_{3}$ and VTE treated $\mathrm{LiTaO}_{3}$ crystals (such as laser-induced photoluminescence and thermoluminescence) can provide the basis for low-cost and efficient methods to determine the uniformity of wafers prior to the fabrication of electro-optic devices.

We have also studied the production and thermal decay of optically active point defects in undoped $\mathrm{KD}_{2} \mathrm{PO}_{4}$ crystals. By irradiating a crystal at $77 \mathrm{~K}$ with $\mathrm{x}$ rays and then temporarily holding it at that temperature, we were able to produce the point defects and "freeze" then into the lattice. We then studied them by both EPR and optical absorption as the crystal was warmed. During the $\mathrm{x}$ ray irradiation of $\mathrm{KD}_{2} \mathrm{PO}_{4}$ crystal at $77 \mathrm{~K}$, large numbers of "free" electrons and holes are formed. These holes will immediately become 
localized within the lattice by forming the $\left[\mathrm{D}_{2} \mathrm{PO}_{4}\right]^{0}$ self-trapped hole centers. This also allows us to assign the two optical absorption bands, at 390 and $550 \mathrm{~nm}$, to the $\left[\mathrm{D}_{2} \mathrm{PO}_{4}\right]^{0}$ centers because these peaks dominate the visible and near-ultraviolet regions of the absorption spectrum taken immediately after the $77 \mathrm{~K}$ irradiation. The EPR data also verifies that deuterium and hydrogen atoms are produced by the initial $77 \mathrm{~K}$ irradiation, and allows us to assign the optical absorption band at $230 \mathrm{~nm}$ to deuterium (and hydrogen) atoms because this band is the remaining dominant peak in the absorption spectrum taken immediately after the $77 \mathrm{~K}$ irradiation. We suggest that this absorption represents the localized transfer of an electron from a neighboring substitutional oxygen ion to the interstitial deuterium atom.

Thermal anneals from $80 \mathrm{~K}$ to room temperature allowed us to monitor the decay of the initial electron and hole traps and to observe the migration of a portion of these charges to new trapping sites. The $\left[\mathrm{DPO}_{4}\right]^{-}$EPR signal and the absorption band at $450 \mathrm{~nm}$ have nearly identical thermal decay characteristics, i.e., both have disappeared when the crystal reaches $260 \mathrm{~K}$. For these reasons, we assign the $450 \mathrm{~nm}$ absorption band to the vacancy-associated $\left[\mathrm{DPO}_{4}\right]^{-}$centers. As the $\left[\mathrm{DPO}_{4}\right]^{-}$centers decay in the 200 to $240 \mathrm{~K}$ region, a portion of these released holes migrate to the silicon trapping sites. The resulting silicon-hole centers are then stable to near room temperature. Electron traps also evolve as the crystal is warmed and at room temperature, $\left(\mathrm{PO}_{3}\right)^{2-}$ oxygen vacancy electron centers are stable for many hours.

The last part of this dissertation deals with results obtained from modeling several important point defects in $\mathrm{KH}_{2} \mathrm{PO}_{4}$ crystals and comparison with experimental data from electron paramagnetic resonance measurements. The quantum-mechanical calculations were performed using Gaussian 98 and graphical outputs from these computations were provided by GaussView. We have chosen Hartree-Fock (HF) as theoretical method and several basis sets of Gaussian functions were used for these calculations. The Gaussian calculations that I have performed for this study were single point energy calculations and geometry optimizations. The primary results of interest were the minimum energy, the isotropic Fermi 
contact coupling terms, and the lattice relaxation that takes place when a point defect is present in the lattice.

A cluster of 41 atoms containing five $\mathrm{PO}_{4}$ units connected by and terminated with hydrogen atoms was used for calculations. The point defects that were model are the selftrapped hole, the proton vacancy, the silicon hole, and the oxygen vacancy. In order to model the various point defects in the KDP lattice, we have used the same cluster of atoms but we made several changes in the input of the Gaussian calculation such as charge, spin multiplicity, molecule specifications, and others, that are specific for each point defect.

The EPR results of hyperfine splitting from experiments were compared with the Fermi contact terms from Gaussian calculations. Excellent agreement between experiment and calculations (with a difference of less than 10\%) was obtained for silicon hole center and for oxygen vacancy electron center. These two point defects are known, from EPR experiments, to be stable at room temperature and our calculations were performed using the crystal structure of KDP at room temperature. For the other two point defects, the difference between the experiment and calculation was larger. Part of this relatively large difference comes from the fact that the self trapped hole and the proton vacancy centers are more stable at low temperature, below Curie temperature, were the crystal structure is slightly different from the structure used for calculations. Nevertheless, for both point defects, the relative values of the Fermi contact terms of the atoms in the cluster reflect the accepted model proposed by EPR measurements and we consider this a very good qualitative result of the Gaussian calculations. 


\section{REFERENCES}

1. C. C. Davis, Lasers and Electro-Optics, (Cambridge University Press, 2000).

2. S. W. S. McKeever, Thermoluminescence of solids, (Cambridge University Press, (1985).

3. D. Xue, K. Betzler, H. Hesse, Solid State Communications 115, 581-585, (2000).

4. Yariv Amnon, Optical electronics in modern communications, (The Oxford Series in Electrical and Computer Engineering, 1997).

5. V. D. Dmitriev, G. G. Gurzadyan, and D. N. Nikogosyan, Handbook of Nonlinear Optical Crystals, (Springer, Series on Optical Sciences, Vol. 64, New York, 1997).

6. H. D. Megaw, Crystal Structures: A Working Approach, (W. B. Saunders Company, 1973).

7. A. M. Prokhorov and Yu S. Kuz'minov, Physics and Chemistry of Crystalline Lithium Niobate, (Adam Hilger Series on Optics and Optoelectronics, 1990).

8. A. Rauber, in Current Topics in Science, edited by E. Kaldis (North-Holland Publishing Company, 1978), Vol. 1, pp. 481-601.

9. A. Garcia-Cabanes, J.A. Sanz-Garcia, J.M. Cabrera, F. Agullo-Lopez, C. Zaldo, R. Pareja, K. Polgar, K. Raksanyi, and I. Folvari, Phys. Rev. B 37, 6085 (1988).

10. D.M. Krol, G. Blasse, and R.C. Powell, J. Chem. Phys. 73, 163 (1980).

11. L. Arizmendi, J.M. Cabrera, and F. Agullo-Lopez, Solid State Commun. 40, 583 (1981).

12. K.L. Sweeney, L.E. Halliburton, D.A. Bryan, R.R. Rice, R. Gerson, and H.E. Tomaschke, J. Appl. Phys. 57, 1036 (1985).

13. F. Klose, M. Wohlecke, and S. Kapphan, Ferroelectrics 92, 181 (1989).

14. C. Fisher, M. Wohlecke, T. Volk, and N. Rubinina, Phys. Status Solidi A 137, 247 (1993). 
15. A.A. Blistanov, V.M. Lyubchenko, and A.N. Goryunova, Crystallography Reports 43, 78 (1998).

16. R.C. Powell and E.E. Freed, J. Chem. Phys. 70, 4681 (1979).

17. A.M. Glass, J. Chem. Phys. 50, 1501 (1969).

18. H. Tsuya, J. Appl. Phys. 46, 4323 (1975).

19. R.M. Khachatryan, A.K. Petrosyan, and E.G. Sharoyan, Soviet Physics-Solid State 27, 1626 (1985).

20. H.J. Donnerberg and O.F. Schirmer, Solid State Commun. 63, 29 (1987).

21. H. Sothe, L.G. Rowan, and J.M. Spaeth, J. Phys.: Condens. Matter 1, 3591 (1989).

22. O. Thiemann, H. Donnerberg, M. Wohlecke, and O.F. Schirmer, Phys. Rev. B 49, 5845 (1994).

23. S.W. Ahn, C. Rudowicz, S.H. Choh, and S. Han, J. Korean Phys. Soc. 30, 99 (1997).

24. M. Grinberg, I. Sokolska, S. Kuck, and W. Jaskolski, Phys. Rev. B 60959 (1999).

25. S.M. Kaczmarek, M. Swirkowicz, R. Jablonski, T. Lukasiewicz, and M. Kwasny, J. Alloys Comp. 300-301, 322 (2000).

26. W. Ryba-Romanowski, P.J. Deren, S. Golab, G. Dominiak-Dzik, J. Appl. Phys. 88, 6078 (2000).

27. M. Grinberg, J. Barzowska, Y.R. Shen, and K.L. Bray, Phys. Rev. B 63,

28. S. Klauer and M. Wohlecke, Phys. Rev. B 49, 158 (1994).

29. A. Grone and S. Kapphan, J. Phys.: Condens. Matter 7, 3051 (1995).

30. M. Wierschem, C. Baumer, A. Jovanovic, M. Wohlecke, S. Kapphan, and L. Kovacs, Phys. Stat. Sol. (b) 215, R7 (1999).

31. C.Y. Chen, K.L. Sweeney, and L.E. Halliburton, Phys. Stat. Sol. (a) 81, 253 (1984).

32. L.A. Kappers, K.L. Sweeney, L.E. Halliburton, and J.H.W. Liaw, Phys. Rev. B 31, 6792 (1985).

33. K.L.Sweeney, L.E. Halliburton, and L.A. Kappers, Phys. Lett. A 116, 81 (1986).

34. A. Garcia-Cabanes, C. Prieto, and L. Arizmendi, Phys. Stat. Sol. (a) 100, K9 (1987). 
35. A. Garcia-Cabanes and C. Prieto, Ferroelectrics 80, 941 (1988).

36. R. Gonzalez, R. Hantehzadeh, C.Y. Chen, L.E. Halliburton, and Y. Chen, Phys. Rev. B 39, 1302 (1989).

37. T.R. Volk, M.A. Ivanov, N.M. Rubinina, A.I. Kholodnykh, and H. Metz, Ferroelectrics, 95, 121 (1989).

38. O.F. Schirmer, O. Thiemann, and M. Wohlecke, J. Phys. Chem. Solids, 52, 185, (1991).

39. F. Abdi, M. Aillerie, P. Bourson, M. D. Fontana, and K. Polgar, J. Appl. Phys., 84, $2251,(1998)$.

40. P. F. Bordui, R.G. Norwood, D.H. Jundt, and M.M. Fejer, J. Appl. Phys., 71, 875 (1991).

41. Yutaka Ohmori, Yoshiyuki Yasojima, and Yoshio Inuishi, Japan. J. Appl. Phys. 14, 1291 (1975).

42. L. Kovacs, Zs. Szaller, I. Cravero, I. Foldvari, and C. Zaldo, J. Phys. Chem. Solids 51, 417 (1990).

43. A. Forster, S. Kapphan, and M. Wohlecke, Phys. Stat. Sol. (b) 143, 755 (1987).

44. T. Volk, M. Wohlecke, N. Rubinina, N. V. Razumovski, F. Jermann, C. Fisher, and R. Bower, Appl. Phys. A 60, 217 (1995).

45. O. F. Schirmer and D. von der Linde, Appl. Phys. Lett. 33, 35 (1978).

46. John E. Wertz and James R. Bolton, Electron Spin Resonance: Elementary Theory and Practical Applications, (McGraw-Hill Book Company, 1972).

47. M. G. Clark, F. J. DiSalvo, A. M. Glass, and G. E. Peterson, J. Chem. Phys. 59, 6209 (1973).

48. H. Kurz, E. Kratzig, W. Keune, H. Engelmann, U. Gonser, B. Dischler, and A. Rauber, Appl. Phys. 12, 355 (1977).

49. G. Malovichko, V. Grachev, and O. Schirmer, Appl. Phys. B 68, 785 (1999). 
50. L. E. Halliburton, K. L. Sweeney, and C. Y. Chen, Nucl. Instrum. Meth. Phys. Res. B 1, 344 (1984).

51. I. M. Zaritskii, L. G. Rakitina, and K. Polgar, Phys. Solid State 37, 1073 (1995).

52. A. L. Alexandrovski, K. Kitamurea, E. Suzuki, L. E. Myers, R. K. Route, and M. M. Fejer, Proc. SPIE, Vol. 3610, pp.44-51 (1999).

53. M. M. Chirila, N. Y. Garces, L. E. Halliburton, D.R. Evans, S.A. Basun, R.S. Meltzer, W. M. Yen, S. A. Rutkowski, D. Shumov, and J. S. Cahill, J. Appl. Phys. 92, 1221 (2002).

54. V. A. Vazhenin, V. B. Guseva, M. Yu. Artyomov, R.K. Route, M.M. Fejer, and R.L. Byer, J. Phys.: Condensed Matter 15, 275 (2003).

55. J. E. Davis, R. S. Hughes, and H. W. Lee, Chem. Phys. Lett. 207, 540 (1993).

56. C. D. Marshall, S. A. Payne, M.A. Henesian, J. A. Speth, and H. T. Powell, J. Opt. Soc. Am. B 11, 774 (1994).

57. S. G. Demos, M. Yan, M. Staggs, J. J. de Yoreo, and H. B. Radousky, Appl. Phys. Lett. 72, 2367 (1998).

58. W. E. Hughes and W. G. Moulton, J. Chem. Phys. 39, 1359 (1963).

59. J. A. McMillan and J. M. Clemens, J. Chem. Phys. 68, 3627 (1978).

60. J. W. Wells, E. Budzinski, and H. C. Box, J. Chem. Phys. 85, 6340 (1986)

61. S. D. Setzler, K. T. Stevens, L. E. Halliburtion, M. Yan, N. P. Zaitseva, and J. J. DeYereo, Phys. Rev. B 57, 2643 (1998).

62. K. T. Stevens, N. Y. Garces, L. E. Halliburton, M. Yan, N. P. Zaitseva, J. J. DEYereo, G. C. Catella, and J. R. Luken, Appl. Phys. Lett. 75, 1503 (1999).

63. E. Dieguez and J. M. Cabrera, J. Phys. D 14, 91 (1981).

64. E. Dieguez, J. M. Cabrera, and F. Agullo-Lopez, J. Chem. Phys. 81, 3369 (1984).

65. Du Varney R C and Kohin R P 1968 Phys. Rev. Lett. 20259.

66. N. Y. Garces, K. T. Stevens, L. E. Halliburton, S. G. Demos, H. B. Radousky, and N. P. Zaitseva, J. Appl. Phys. 89, 47 (2001). 
67. K. Fujioka, S. Matsuo, T. Kanabe, H. Fujita, and M. Nakatsuka, J. Crystal Growth 181, 265 (1997).

68. E. Dieguez, A. Cintas, P. Hernandez, and J. M. Cabrera, J. Crystal Growth 73, 193 (1985).

69. N. Y. Garces, K. T. Stevens, L. E. Halliburton, M. Yan, N. P. Zaitseva, J. J. DeYero, J. of Crystal Growth 225, 435 (2001).

70. Gaussian 98 (Revision A.1), M. J. Frisch, G. W. Trucks, H. B. Schlegel, G. E. Scuseria, M.A. Robb, J. R. Cheeseman, V. G. Zakrzewski, J. A. Montgomery, R. E. Stratmann, J. C.Burant, S. Dapprich, J. M. Millam, A. D. Daniels, K. N. Kudin, M. C. Strain, O. Farkas,J. Tomasi, V. Barone, M. Cossi, R. Cammi, B. Mennucci, C. Pomelli, C. Adamo, S.Clifford, J. Ochterski, G. A. Petersson, P. Y. Ayala, Q. Cui, K. Morokuma, D. K. Malick,A. D. Rabuck, K. Raghavachari, J. B. Foresman, J. Cioslowski, J. V. Ortiz, B. B. Stefanov, G. Liu, A. Liashenko, P. Piskorz, I. Komaromi, R. Gomperts, R. L. Martin, D. J. Fox, T. Keith, M. A. Al-Laham, C. Y. Peng, A. Nanayakkara, C. Gonzalez, M. Challacombe, P. M. W. Gill, B. G. Johnson, W. Chen, M. W. Wong, J. L. Andres, M. Head-Gordon, E. S. Replogle, and J. A. Pople, Gaussian, Inc., Pittsburgh, PA, 1998.

71. John P. Lowe, Quantum chemistry, (Academic Press, 1978).

72. Gianfranco Pacchioni, Fabiano Frigoli, Davide Ricci, and John A. Weil, Phys. Rev. B, 63, 054102/1-8, (2000).

73. W. E. Hagston, Phys. Status Solidi (b) 47, 281 (1971).

74. Th. Hangleiter and J. M. Spaeth, Solid State Commun. 35, 23 (1980).

75. B. K. Myer and J. M. Spaeth, J. Phys. C: Solid State Physics 17, 2213 (1984).

76. A. V. Egranov and B. P. Chernyago, Phys. Status Solidi (b) 188, 615 (1995). 